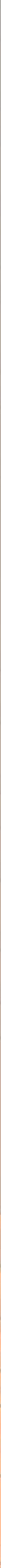




\title{
Moduli of elliptic curves via twisted stable maps
}

\author{
Andrew Niles
}

\begin{abstract}
Abramovich, Corti and Vistoli have studied modular compactifications of stacks of curves equipped with abelian level structures arising as substacks of the stack of twisted stable maps into the classifying stack of a finite group, provided the order of the group is invertible on the base scheme. Recently Abramovich, Olsson and Vistoli extended the notion of twisted stable maps to allow arbitrary base schemes, where the target is a tame stack, not necessarily Deligne-Mumford. We use this to extend the results of Abramovich, Corti and Vistoli to the case of elliptic curves with level structures over arbitrary base schemes; we prove that we recover the compactified Katz-Mazur regular models, with a natural moduli interpretation in terms of level structures on Picard schemes of twisted curves. Additionally, we study the interactions of the different such moduli stacks contained in a stack of twisted stable maps in characteristics dividing the level.
\end{abstract}

1. Introduction

2. Review of moduli of generalized elliptic curves 2144

3. Generalities/review of twisted stable maps 2152

4. Moduli of elliptic curves in $\overline{\mathscr{K}}_{1,1}\left(\mathscr{B} \mu_{N}\right) \quad 2165$

5. Moduli of elliptic curves in $\overline{\mathscr{K}}_{1,1}\left(\mathscr{B} \mu_{N}^{2}\right) \quad 2182$

6. Comparison with the classical moduli stacks 2191

7. Other compactified moduli stacks of elliptic curves 2197

Appendix: On moduli of curves of higher genus $\quad 2199$

Acknowledgements 2200

References $\quad 2200$

\section{Introduction}

Abramovich and Vistoli [2002] introduced the stack $\mathcal{K}_{g, n}(\mathscr{X})$ of $n$-pointed genus- $g$ twisted stable maps into $\mathscr{X}$, where $\mathscr{X}$ is a proper tame Deligne-Mumford stack over a base scheme $S$ with a projective coarse moduli space $X / S$. They proved that

MSC2010: primary 11G18; secondary 14K10, 14H10, 14D23, $14 \mathrm{H} 52$.

Keywords: generalized elliptic curve, twisted curve, Drinfeld structure, moduli stack. 
$\mathscr{K}_{g, n}(\mathscr{X})$ is an algebraic stack, proper over the Kontsevich stack $\bar{M}_{g, n}(X)$ of stable maps into $X$, giving an appropriate analogue of the usual Kontsevich stack of stable maps when the target is allowed to be a tame stack instead of merely a scheme or algebraic space. In particular, taking $\mathscr{X}=\mathscr{B} G$ for a finite group $G$ naturally yields a modular compactification of the stack of $n$-pointed genus- $g$ curves with certain level structure, where the level structure on a curve corresponds to giving a $G$-torsor over the curve; this is studied extensively in [Abramovich et al. 2003]. This of course differs from the approach in [Abramovich and Romagny 2012], where the coverings of curves are assumed themselves to be stable curves; here the coverings are generally not stable curves. In [Petersen 2012] it is shown that in good characteristics we recover the usual compactification of $y(N)$ in $\mathscr{K}_{1,1}\left(\mathscr{B}(\mathbb{Z} /(N))^{2}\right)$.

However, the algebraic stack $\mathscr{B} G$ is not tame in characteristics dividing $|G|$, so a priori the results of [Abramovich et al. 2003] only hold over $\mathbb{Z}[1 /|G|]$. The notion of a tame stack is generalized in [Abramovich et al. 2008b] to algebraic stacks that are not necessarily Deligne-Mumford, and in [Abramovich et al. 2011] the stack $\mathscr{K}_{g, n}(\mathscr{X})$ of twisted stable maps into certain proper tame algebraic stacks is introduced and shown to be a proper algebraic stack. This naturally leads us to attempt replacing the finite group $G$ with a group scheme $\mathscr{G}$, agreeing with $G$ over $\mathbb{Z}[1 /|G|]$ but such that $\mathscr{B} \mathscr{G}$ is a tame algebraic stack over $\operatorname{Spec}(\mathbb{Z})$; see, for example, [Abramovich 2012]. In particular, the group scheme $\mu_{N}$ is (noncanonically) isomorphic to $\mathbb{Z} /(N)$ over $\mathbb{Z}\left[1 / N, \zeta_{N}\right]$, and the classifying stack $\mathscr{B} \mu_{N}$ (unlike $\mathscr{B}(\mathbb{Z} /(N))$ ) is a tame stack over $\operatorname{Spec}(\mathbb{Z})$.

The purpose of this paper is to record how the results of [Abramovich et al. 2003] extend to moduli of elliptic curves with level structure over arbitrary base schemes, using the group scheme $\mu_{N}$ in place of $\mathbb{Z} /(N)$. For example, the moduli stack $y_{1}(N)$ arises as an open substack in the rigidification $\overline{\mathscr{K}}_{1,1}\left(\mathscr{B} \mu_{N}\right)$ (defined below) of $\mathscr{K}_{1,1}\left(\mathscr{B} \mu_{N}\right)$. Explicitly, given an elliptic curve $E / S$ and a $\left[\Gamma_{1}(N)\right]$-structure $P$ on $E$, via the canonical group scheme isomorphism $E \cong \operatorname{Pic}_{E / S}^{0}$ over $S$, we may view $P$ as a "point of exact order $N$ " on $\mathrm{Pic}_{E / S}^{0}$, determining a group scheme homomorphism $\phi: \mathbb{Z} /(N) \rightarrow \mathrm{Pic}_{E / S}^{0}$. The stack $\frac{\mathscr{K} /}{\mathscr{K}_{1,1}}\left(\mathscr{B} \mu_{N}\right)$ will be seen to classify certain pairs $(\mathscr{C}, \phi)$ where $\mathscr{C} / S$ is a 1 -marked genus-1 twisted stable curve and $\phi: \mathbb{Z} /(N) \rightarrow$ $\mathrm{Pic}_{\mathscr{C} / S}^{0}$ is a group scheme homomorphism, so this construction defines the immersion $y_{1}(N) \rightarrow \overline{\mathscr{K}}_{1,1}\left(\mathscr{B} \mu_{N}\right)$. We will define the notion of a $\left[\Gamma_{1}(N)\right]$-structure on a $1-$ marked genus- 1 twisted stable curve, and write $X_{1}^{\mathrm{tw}}(N)$ for the stack classifying such structures. Our first main result is:

Theorem 1.1. Let $S$ be a scheme and let $X_{1}^{\mathrm{tw}}(N)$ be the stack over $S$ classifying $\left[\Gamma_{1}(N)\right]$-structures on 1-marked genus-1 twisted stable curves with nonstacky marking. Then $X_{1}^{\mathrm{tw}}(N)$ is a closed substack of $\overline{\mathscr{K}}_{1,1}\left(\mathscr{B} \mu_{N}\right)$, which contains $y_{1}(N)$ as an open dense substack. 
This will be proved as Theorem 4.6. The main point is to verify the valuative criterion for properness. We accomplish this by using properness of $X_{1}(N)$ and the $e_{N}$-pairing on generalized elliptic curves. To complete a family of twisted curves with level structure when the generic fiber is smooth, we first complete it to a family of generalized elliptic curves with level structure. Then we use a quotient construction involving the $e_{N}$-pairing to produce a completed family of twisted curves with level structure, modifying our strategy in characteristics dividing $N$ by exploiting the relationship between cyclotomic torsors and line bundles (Lemma 3.19). The same techniques immediately give a corresponding result for $y(N)$, which is an open substack of $\overline{\mathscr{K}}_{1,1}\left(\mathscr{B} \mu_{N}^{2}\right)$; its closure $X^{\mathrm{tw}}(N)$ classifies $[\Gamma(N)]$-structures on 1-marked genus-1 twisted stable curves, as we show in Theorem 5.3.

Unsurprisingly, it turns out that these closures are isomorphic as algebraic stacks to the stacks $X_{1}(N)$ and $X(N)$ classifying $\left[\Gamma_{1}(N)\right]$-structures and $[\Gamma(N)]$-structures on generalized elliptic curves, as studied in [Conrad 2007]:

Theorem 1.2. Over the base $S=\operatorname{Spec}(\mathbb{Z})$, there is a canonical isomorphism of algebraic stacks $X_{1}^{\mathrm{tw}}(N) \cong X_{1}(N)$ extending the identity map on $Y_{1}(N)$, and a canonical isomorphism of algebraic stacks $X^{\mathrm{tw}}(N) \cong X(N)$ extending the identity map on $y(N)$.

We will prove this as Theorem 6.1. We first verify that $X_{1}^{\mathrm{tw}}(N)$ and $X^{\mathrm{tw}}(N)$ are finite over $\bar{M}_{1,1}$. Some commutative algebra then tells us they are CohenMacaulay over $\operatorname{Spec}(\mathbb{Z})$, at which point we may proceed as in [Conrad 2007, $\S 4.1]$ to identify these stacks with the normalizations of $\bar{M}_{1,1}$ in $\left.X_{1}(N)\right|_{\mathbb{Z}[1 / N]}$ and $\left.X(N)\right|_{\mathbb{Z}[1 / N]}$. These isomorphisms have a natural moduli interpretation, as discussed in Corollaries 6.9 and 6.10 .

The techniques of this paper also yield new moduli interpretations of various moduli stacks of elliptic curves that are not (apparently) contained in a stack of twisted stable maps. These results may be known to experts, but are not recorded in the literature; for completeness we include a careful proof of the modular interpretation of the closure of $y_{1}(N)$ in $\overline{\mathscr{K}}_{1,1}\left(\mathscr{B}_{\mu_{N}}\right)$. We also study how the different moduli stacks of elliptic curves contained in $\overline{\mathscr{K}}_{1,1}\left(\mathscr{B} \mu_{N}\right)$ and $\overline{\mathscr{K}}_{1,1}\left(\mathscr{B} \mu_{N}^{2}\right)$ interact in characteristics dividing $N$; this easily generalizes an example in [Abramovich et al. 2011] but otherwise does not appear in the literature.

In the Appendix we recall an example of [Chai and Norman 1990] which implies that the techniques of this paper do not generalize nicely to curves of higher genus: using the Katz-Mazur notion of a "full set of sections" we can define a stack over $\operatorname{Spec}(\mathbb{Z})$ classifying genus- $g$ curves with full level- $N$ structures on their Jacobians, but this stack is not even flat over $\operatorname{Spec}(\mathbb{Z})$. The corresponding stack over $\mathbb{Z}[1 / N]$ is well-behaved, and arises as an open substack of $\overline{\mathscr{K}}_{g, 0}\left(\mathscr{B}_{\mu_{N}^{2 g}}^{2 g}\right.$, but no moduli interpretation for its closure in characteristics dividing $N$ appears to be known. 


\section{Review of moduli of generalized elliptic curves}

Drinfeld level structures on generalized elliptic curves. For the convenience of the reader, we recall the definitions and results from the theory of generalized elliptic curves that we require in this paper.

Definition 2.1. A Deligne-Rapoport (DR) semistable curve of genus 1 over a scheme $S$ is a proper, flat, separated, finitely presented morphism of schemes $f: C \rightarrow S$, all of whose geometric fibers are nonempty, connected semistable curves with trivial dualizing sheaves.

By [Deligne and Rapoport 1973, §II.1], an equivalent definition for $f: C \rightarrow S$ to be a DR semistable curve of genus 1 is that $f$ is a proper flat morphism of finite presentation and relative dimension 1 , such that every geometric fiber is either a smooth connected genus-1 curve or a Néron polygon. Recall [loc. cit.] that over a base scheme $S$, the standard Néron $N$-gon $C_{N} / S$ (for any $N \geq 1$ ) is obtained from $\widetilde{C}_{N}:=\mathbb{P}_{S}^{1} \times \mathbb{Z} /(N)$ by "gluing" the section 0 in the $i$-th copy of $\mathbb{P}_{S}^{1}$ to the section $\infty$ in the $(i+1)$-th copy of $\mathbb{P}_{S}^{1}$ :

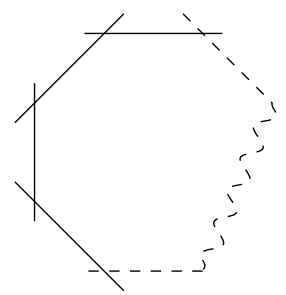

Standard Néron $N$-gon

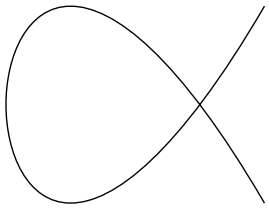

Standard Néron 1-gon

The natural multiplication action of $\mathbb{G}_{m}$ on $\mathbb{P}_{S}^{1}$, together with the action of $\mathbb{Z} /(N)$ on itself via the group law, determines an action of the group scheme $\mathbb{G}_{m} \times \mathbb{Z} /(N)$ on $\mathbb{P}_{S}^{1} \times \mathbb{Z} /(N)$, descending uniquely to an action of $\mathbb{G}_{m} \times \mathbb{Z} /(N)=C_{N}^{\mathrm{sm}}$ on $C_{N}$ [Deligne and Rapoport 1973, II.1.9].

Definition 2.2. A generalized elliptic curve over a scheme $S$ is a DR semistable curve $E / S$ of genus 1, equipped with a morphism $E^{\mathrm{sm}} \times E \rightarrow E$ and a section $0_{E} \in E^{\mathrm{sm}}(S)$ such that the restriction $E^{\mathrm{sm}} \times E^{\mathrm{sm}} \rightarrow E^{\mathrm{sm}}$ makes $E^{\mathrm{sm}}$ a commutative group scheme over $S$ with identity $0_{E}$, and such that on any singular geometric fiber $E_{\bar{S}}$, the translation action by a rational point on $E_{\bar{s}}^{\mathrm{sm}}$ acts by a rotation on the graph $\Gamma\left(E_{\bar{s}}\right)$ [Deligne and Rapoport 1973, I.3.5] of the irreducible components of $E_{\bar{s}}$.

By [Deligne and Rapoport 1973, II.1.15], over an algebraically closed field a generalized elliptic curve is either a smooth elliptic curve or a Néron $N$-gon (for some $N \geq 1$ ) with the action described above. In fact this result says more: for any generalized elliptic curve $E / S$, there is a locally finite family $\left(S_{N}\right)_{N \geq 1}$ of closed 
subschemes of $S$ such that $\bigcup S_{N}$ is the nonsmooth locus in $S$ of $E \rightarrow S$, and $E \times{ }_{S} S_{N}$ is fppf locally on $S_{N}$ isomorphic to the standard Néron $N$-gon over $S_{N}$.

Recall that for an $S$-scheme $X$, a relative effective Cartier divisor in $X$ over $S$ is an effective Cartier divisor in $X$ which is flat over $S$.

Definition 2.3. Let $E / S$ be a generalized elliptic curve. A $\left[\Gamma_{1}(N)\right]$-structure on $E$ is a section $P \in E^{\mathrm{sm}}(S)$ such that:

- $N \cdot P=0_{E}$.

- The relative effective Cartier divisor

$$
D:=\sum_{a \in \mathbb{Z} /(N)}[a \cdot P]
$$

in $E^{\mathrm{sm}}$ is a subgroup scheme.

- For any geometric point $\bar{p} \rightarrow S, D_{\bar{p}}$ meets every irreducible component of $E_{\bar{p}}$. We write $X_{1}(N)$ for the stack over $\operatorname{Spec}(\mathbb{Z})$ associating to a scheme $S$ the groupoid of pairs $(E, P)$, where $E / S$ is a generalized elliptic curve and $P$ is a $\left[\Gamma_{1}(N)\right]$-structure on $E$. We write $y_{1}(N)$ for the substack classifying such pairs, where $E / S$ is a smooth elliptic curve.

Definition 2.4. Let $E / S$ be a generalized elliptic curve. A $[\Gamma(N)]$-structure on $E$ is an ordered pair $(P, Q)$ of sections in $E^{\mathrm{sm}}[N](S)$ such that:

- The relative effective Cartier divisor

$$
D:=\sum_{a, b \in \mathbb{Z} /(N)}[a \cdot P+b \cdot Q]
$$

in $E^{\mathrm{sm}}$ is an $N$-torsion subgroup scheme, hence $D=E^{\mathrm{sm}}[N]$.

- For any geometric point $\bar{p} \rightarrow S, D_{\bar{p}}$ meets every irreducible component of $E_{\bar{p}}$. We write $X(N)$ for the stack over $\operatorname{Spec}(\mathbb{Z})$ associating to a scheme $S$ the groupoid of tuples $(E,(P, Q))$, where $E / S$ is a generalized elliptic curve and $(P, Q)$ is a $[\Gamma(N)]$-structure on $E$. We write $y(N)$ for the substack classifying such tuples, where $E / S$ is a smooth elliptic curve.

Definition 2.5. Let $E / S$ be a generalized elliptic curve, and let $G$ be a 2-generated finite abelian group, say $G \cong \mathbb{Z} /\left(n_{1}\right) \times \mathbb{Z} /\left(n_{2}\right)$, with $n_{2} \mid n_{1}$. A $G$-structure on $E$ is a homomorphism $\phi: G \rightarrow E^{\mathrm{sm}}$ of group schemes over $S$ such that:

- The relative effective Cartier divisor

$$
D:=\sum_{a \in G}[\phi(a)]
$$

in $E^{\mathrm{sm}}$ is an $n_{1}$-torsion subgroup scheme. 
- For every geometric point $\bar{p} \rightarrow S, D_{\bar{p}}$ meets every irreducible component of $E_{\bar{p}}$.

Theorem 2.6 [Conrad 2007, 3.1.7, 3.2.7, 3.3.1, 4.1.1]. $X_{1}(N)$ and $X(N)$ are regular Deligne-Mumford stacks, proper and flat over $\operatorname{Spec}(\mathbb{Z})$ of pure relative dimension 1.

In particular, it follows (see [Conrad 2007, 4.1.5]) that $X_{1}(N)\left(\operatorname{resp} . X_{(N)}\right)$ is canonically identified with the normalization of $\bar{M}_{1,1}$ in the normal DeligneMumford stack $\left.X_{1}(N)\right|_{\mathbb{Z}[1 / N]}\left(\operatorname{resp} .\left.X(N)\right|_{\mathbb{Z}[1 / N]}\right)$, as in [Deligne and Rapoport 1973, §VII.2; Katz and Mazur 1985, §8.6].

Reductions mod $\boldsymbol{p}$ of the moduli stacks. It will be useful for us to have a description of the "reduction mod $p$ " of the stacks $X_{1}(N)$ and $X(N)$ for primes $p$ dividing $N$. These reductions are described using Katz and Mazur's "crossings theorem", which we now recall.

We work over a fixed field $k$. Let $Y / k$ be a smooth curve, and let $X \rightarrow Y$ be finite and flat. Suppose there is a nonempty finite set of $k$-rational points of $Y$ (which we will call the supersingular points of $Y$ ) such that for each supersingular point $y_{0}$, $X_{y_{0}}$ is a single $k$-rational point, and $\widehat{O}_{X, x_{0}} \cong k \llbracket x, y \rrbracket /(f)$ for some $f$ (depending on $\left.y_{0}\right)$.

Also suppose we have a finite collection of closed immersions $\left\{Z_{i} \hookrightarrow X\right\}_{i=1}^{n}$, where each $Z_{i}$ is finite and flat over $Y$, with $Z_{i}^{\text {red }}$ a smooth curve over $k$, such that the morphism $\sqcup Z_{i} \rightarrow X$ is an isomorphism over the nonsupersingular locus of $Y$, and such that for each $i$ and each supersingular point $y_{0} \in Y,\left(Z_{i}\right)_{y_{0}}$ is a single $k$-rational point.

Theorem 2.7 (crossings theorem, [Katz and Mazur 1985, 13.1.3]). Under the above assumptions, let $y_{0} \in Y$ be a supersingular point and $x_{0}=X_{y_{0}}$. Then

$$
\widehat{\mathrm{O}}_{X, x_{0}} \cong k \llbracket x, y \rrbracket / \prod_{i=1}^{m} f_{i}^{e_{i}},
$$

where each $f_{i}$ is irreducible in $k \llbracket x, y \rrbracket$, each $f_{i}$ and $f_{j}(i \neq j)$ are distinct in $k \llbracket x, y \rrbracket$ modulo multiplication by units, and for $z_{i, 0}:=\left(Z_{i}\right)_{y_{0}}$ we have

$$
\widehat{\mathrm{O}}_{Z_{i}, z_{i, 0}} \cong k \llbracket x, y \rrbracket /\left(f_{i}^{e_{i}}\right) .
$$

If $Y$ is (geometrically) connected then each $Z_{i}$ is (geometrically) connected, in which case $\left\{Z_{i}\right\}_{i=1}^{n}$ is the set of irreducible components of $X$.

Definition 2.8. Under the above assumptions, we say that $X$ is the disjoint union with crossings at the supersingular points of the closed subschemes $\left\{Z_{i}\right\}_{i=1}^{n}$.

If $\mathscr{X} \rightarrow \mathscr{Y}$ is a finite (hence representable) flat morphism of algebraic stacks over k, with $\mathscr{Y}$ Deligne-Mumford (hence $\mathscr{X}$ also Deligne-Mumford), and $\left\{\mathscr{L}_{i} \hookrightarrow \mathscr{X}\right\}_{i=1}^{n}$ 
is a finite collection of closed immersions of algebraic stacks, such that for some étale surjection $Y^{\prime} \rightarrow \mathscr{y}$ with $Y^{\prime}$ a scheme, the algebraic spaces $Y^{\prime}, \mathscr{X} \times \mathscr{y} Y^{\prime}$, and $\left\{\mathscr{L}_{i} \times{ }^{\prime} Y^{\prime}\right\}$ are schemes satisfying the assumptions of the crossings theorem, we say that $\mathscr{X}$ is the disjoint union with crossings at the supersingular points of the closed substacks $\left\{\mathscr{L}_{i}\right\}$.

Remark 2.9. We will solely be applying the above theorem in the case $\mathscr{y}=\overline{\mathcal{M}}_{1,1}$, with $\mathscr{L}$ an algebraic stack known to be quasifinite and proper over $\bar{M}_{1,1} \cdot \bar{M}_{1,1}$ is a Deligne-Mumford stack with separated diagonal, so once we know that $\mathscr{L} \rightarrow \bar{M}_{1,1}$ is representable, it follows from [Knutson 1971, II.6.16] that the algebraic spaces $\mathscr{X} \times \overline{\mathcal{M}}_{1,1} Y^{\prime}$ and $\left\{\mathscr{E}_{i} \times \overline{\bar{M}}_{1,1} Y^{\prime}\right\}$ are schemes.

Definition 2.10. Let $S \in \operatorname{Sch} / \mathbb{F}_{p}$. Let $E / S$ be an elliptic curve, with relative Frobenius $F: E \rightarrow E^{(p)}$. Let $G \subset E$ be a finite, locally free $S$-subgroup scheme of rank $p^{n}, n \geq 1$. For integers $a, b \geq 0$ with $a+b=n$, we say that $G$ is an $(a, b)$-subgroup if $\operatorname{ker}\left(F^{a}\right) \subset G$, and if in the resulting factorization of $E \rightarrow E / G$ as

$$
E \stackrel{F^{a}}{\rightarrow} E^{\left(p^{a}\right)} \stackrel{\pi}{\rightarrow} E / G
$$

we have $\operatorname{ker}(\widehat{\pi})=\operatorname{ker}\left(F_{E / G}^{b}\right)$ (where $\widehat{\pi}$ denotes the dual isogeny and $F_{E / G}: E / G \rightarrow$ $(E / G)^{(p)}$ is the relative Frobenius). In particular note that $E^{\left(p^{a}\right)} \cong(E / G)^{\left(p^{b}\right)}$.

We say that $G$ is an $(a, b)$-cyclic subgroup if it is an $(a, b)$-subgroup, and either $a=0, b=0$, or there exists a closed subscheme $Z \subset S$ defined by a sheaf of ideals $\mathscr{I} \subset O_{S}$, with $\Phi^{p-1}=0$, such that the isomorphism $\left.\left.E^{\left(p^{a}\right)}\right|_{Z} \cong(E / G)^{\left(p^{b}\right)}\right|_{Z}$ is induced by an isomorphism $\left.\left.E^{\left(p^{a-1}\right)}\right|_{Z} \cong(E / G)^{\left(p^{b-1}\right)}\right|_{Z}$.

Finally, a $\left[\Gamma_{1}\left(p^{n}\right)\right]-(a, b)$-cyclic structure on $E$ is a $\left[\Gamma_{1}\left(p^{n}\right)\right]$-structure $P \in$ $E\left[p^{n}\right](S)$ such that the $S$-subgroup scheme

$$
D:=\sum_{m \in \mathbb{Z} /\left(p^{n}\right)}[m \cdot P]
$$

in $E$ is an $(a, b)$-cyclic subgroup of $E$. We write $y_{1}\left(p^{n}\right)_{S}^{(a, b)}$ for the substack of $y_{1}\left(p^{n}\right)_{S}$ associating to a scheme $T / S$ the groupoid of pairs $(E, P)$, where $E / T$ is an elliptic curve and $P$ is a $\left[\Gamma_{1}\left(p^{n}\right)\right]-(a, b)$-cyclic structure on $E$.

If $E$ is an ordinary elliptic curve over a scheme $S$ of characteristic $p$, it is considerably easier to describe what is meant by a $\left[\Gamma_{1}\left(p^{n}\right)\right]$ - $(a, b)$-cyclic structure on $E$. Namely, it is just a $\left[\Gamma_{1}\left(p^{n}\right)\right]$-structure $P$ on $E$ such that the relative effective Cartier divisor

$$
D_{b}:=\sum_{m=1}^{p^{b}}[m \cdot P]
$$

is a subgroup scheme of $E$ which is étale over $S$. Note that for an arbitrary $\left[\Gamma_{1}\left(p^{n}\right)\right]$-structure $P$ on $E, D_{b}$ will not generally be a subgroup scheme of $E$ if 
$b<n$, and even if it is a subgroup scheme it might not be étale over $S$. Being a [ $\left.\Gamma_{1}\left(p^{n}\right)\right]-(a, b)$-cyclic structure on $E$ (for $E$ ordinary) means that for any geometric fiber $E_{\bar{s}}$ (so $E_{\bar{s}}\left[p^{n}\right] \cong \mu_{p^{n}} \times \mathbb{Z} /\left(p^{n}\right)$ over $\left.k(\bar{s})\right) P_{\bar{s}}$ has exact order $p^{b}$ as an element of the group $E_{\bar{s}}\left[p^{n}\right](k(\bar{s})) \cong \mathbb{Z} /\left(p^{n}\right)$.

The following elementary result will be required when we study the interactions in characteristic $p$ of different moduli stacks of elliptic curves contained in a moduli stack of twisted stable maps. We include a proof for lack of a suitable reference.

Lemma 2.11. Let $E / S / \mathbb{F}_{p}$ be an elliptic curve, and let $P$ be $a\left[\Gamma_{1}\left(p^{n}\right)\right]-(a, b)$-cyclic structure on $E$. Then $P$ is a $\left[\Gamma_{1}\left(p^{n+1}\right)\right]$-structure on $E$ and is $\left[\Gamma_{1}\left(p^{n+1}\right)\right]-(a+1, b)$ cyclic.

Proof. Consider the relative effective Cartier divisor

$$
G:=\sum_{m \in \mathbb{Z} /\left(p^{n}\right)}[m \cdot P]
$$

in $E$. This is an $S$-subgroup scheme containing $\operatorname{ker}\left(F^{a}\right)$, and in the resulting factorization of the quotient map $E \rightarrow E / G$ as

$$
E \stackrel{F^{a}}{\rightarrow} E^{\left(p^{a}\right)} \stackrel{\pi}{\rightarrow} E / G
$$

we have $\operatorname{ker}(\widehat{\pi})=\operatorname{ker}\left(F_{E / G}^{b}\right)$. Consider also the relative effective Cartier divisor

$$
G^{\prime}:=\sum_{m \in \mathbb{Z} /\left(p^{n+1}\right)}[m \cdot P]
$$

in $E . G$ is a subgroup of $G^{\prime}$, and the image of $G^{\prime}$ in $E / G$ is the relative effective Cartier divisor

$$
\sum_{m \in \mathbb{Z} /(p)}\left[m \cdot 0_{E / G}\right]
$$

This is simply the kernel of $F_{E / G}: E / G \rightarrow(E / G)^{(p)}$, so we may identify $E / G^{\prime}$ with $(E / G)^{(p)}$ and the quotient map $E / G \rightarrow E / G^{\prime}$ with the relative Frobenius $F_{E / G}: E / G \rightarrow(E / G)^{(p)}$. In particular, the quotient map $E \rightarrow E / G^{\prime}$ is a cyclic $p^{n+1}$-isogeny of elliptic curves with kernel generated by $P$, so we may already conclude that $P$ is a $\left[\Gamma_{1}\left(p^{n+1}\right)\right]$-structure on $E$.

Now, since $G^{\prime}=p \cdot G$ as Cartier divisors, if $\operatorname{ker}\left(F^{a}\right) \subset G$ then $\operatorname{ker}\left(F^{a+1}\right) \subset G^{\prime}$. Factor the quotient map $E \rightarrow E / G^{\prime}=(E / G)^{(p)}$ as

$$
E \stackrel{F^{a+1}}{\rightarrow} E^{\left(p^{a+1}\right)} \stackrel{\pi^{\prime}}{\rightarrow}(E / G)^{(p)} .
$$


We have a diagram

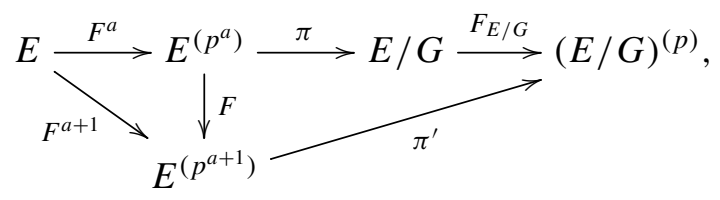

where the composite of the top arrows $E \rightarrow(E / G)^{(p)}$ is the natural quotient map $E \rightarrow E / G^{\prime}$. The outer arrows and the left-hand triangle commute, hence the right-hand triangle commutes as well. Now consider the following diagram:

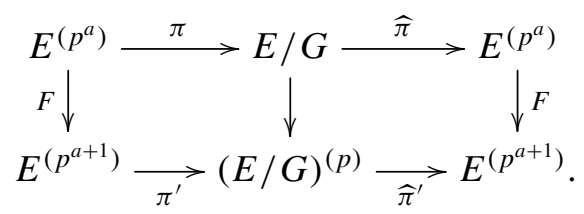

The outer rectangle commutes since the horizontal composites are the isogenies $[\operatorname{deg}(\pi)]$ and $\left[\operatorname{deg}\left(\pi^{\prime}\right)\right]$ (and $\operatorname{deg}(\pi)=\operatorname{deg}\left(\pi^{\prime}\right)$ ), and we have shown that the lefthand square commutes, so the right-hand square commutes as well. Since $\operatorname{ker}(\widehat{\pi})=$ $\operatorname{ker}\left(F_{E / G}^{b}\right)$, we conclude that $\operatorname{ker}\left(\widehat{\pi}^{\prime}\right)=\operatorname{ker}\left(F_{(E / G)^{(p)}}^{b}\right)$.

Finally, suppose $a, b \geq 1$. Since $E^{\left(p^{a}\right)} \cong(E / G)^{\left(p^{b}\right)}$ we also have

$$
E^{\left(p^{a+1}\right)} \cong(E / G)^{\left(p^{b+1}\right)}=\left((E / G)^{(p)}\right)^{\left(p^{b}\right)},
$$

and this is already induced by the isomorphism

$$
E^{\left(p^{a}\right)} \cong(E / G)^{\left(p^{b}\right)}=\left((E / G)^{(p)}\right)^{\left(p^{b-1}\right)} .
$$

Therefore $P$ is a $\left[\Gamma_{1}\left(p^{n+1}\right)\right]-(a+1, b)$-cyclic structure on $E$.

Remark 2.12. The reason the above argument fails when $p$ is invertible on the base scheme $S$ is that, preserving the notation of the above proof, the image of the relative Cartier divisor $G^{\prime}$ in $E / G$ is not a subgroup of $E / G$ unless $S$ is an $\mathbb{F}_{p}$-scheme. Indeed, the image of $G^{\prime}$ in $E / G$ is the relative effective Cartier divisor $p \cdot\left[0_{E / G}\right]$, which is finite flat of rank $p$ over $S$. If $p$ is invertible on $S$, any finite flat commutative group scheme of rank $p$ over $S$ is étale (see [Shatz 1986, Corollary 4.3]), but $p \cdot\left[0_{E / G}\right]$ is obviously not étale.

It is straightforward to extend the definition of a $\left[\Gamma_{1}\left(p^{n}\right)\right]-(a, b)$-cyclic structure, and the result of the above lemma, to the case of generalized elliptic curves. Let $E / S / \mathbb{F}_{p}$ be a generalized elliptic curve; let $S_{1} \subset S$ be the open locus where the morphism $E \rightarrow S$ is smooth, and let $S_{2} \subset S$ be the complement of the closed locus in $S$ where the geometric fibers are supersingular elliptic curves. We say that a $\left[\Gamma_{1}\left(p^{n}\right)\right]$-structure $P$ on $E / S$ is a $\left[\Gamma_{1}\left(p^{n}\right)\right]$ - $(a, b)$-cyclic structure if: 
- $\left.P\right|_{S_{1}}$ is a $\left[\Gamma_{1}\left(p^{n}\right)\right]-(a, b)$-cyclic structure on the elliptic curve $E_{S_{1}} / S_{1}$ and

- the relative effective Cartier divisor

$$
D_{b}:=\sum_{m=1}^{p^{b}}\left[\left.m \cdot P\right|_{S_{2}}\right]
$$

in $\left.E^{\mathrm{sm}}\right|_{S_{2}}$ is a subgroup scheme of $\left.E^{\mathrm{sm}}\right|_{S_{2}}$ which is étale over $S_{2}$.

We write $X_{1}\left(p^{n}\right)_{S}^{(a, b)}$ for the substack of $X_{1}\left(p^{n}\right)_{S}$ associating to a scheme $T / S$ the groupoid of pairs $(E, P)$, where $E / T$ is a generalized elliptic curve and $P$ is a $\left[\Gamma_{1}\left(p^{n}\right)\right]-(a, b)$-cyclic structure on $E$.

Theorem 2.13 [Katz and Mazur 1985, 13.5.4]. Let $k$ be a perfect field of characteristic $p . y_{1}\left(p^{n}\right)_{k}$ (resp. $\left.X_{1}\left(p^{n}\right)_{k}\right)$ is the disjoint union, with crossings at the supersingular points, of the $n+1$ substacks $y_{1}\left(p^{n}\right)_{k}^{(a, n-a)}\left(\right.$ resp. $\left.X_{1}\left(p^{n}\right)_{k}^{(a, n-a)}\right)$ for $0 \leq a \leq n$.

It can be helpful to visualize $X_{1}\left(p^{n}\right)_{k}$ as follows:

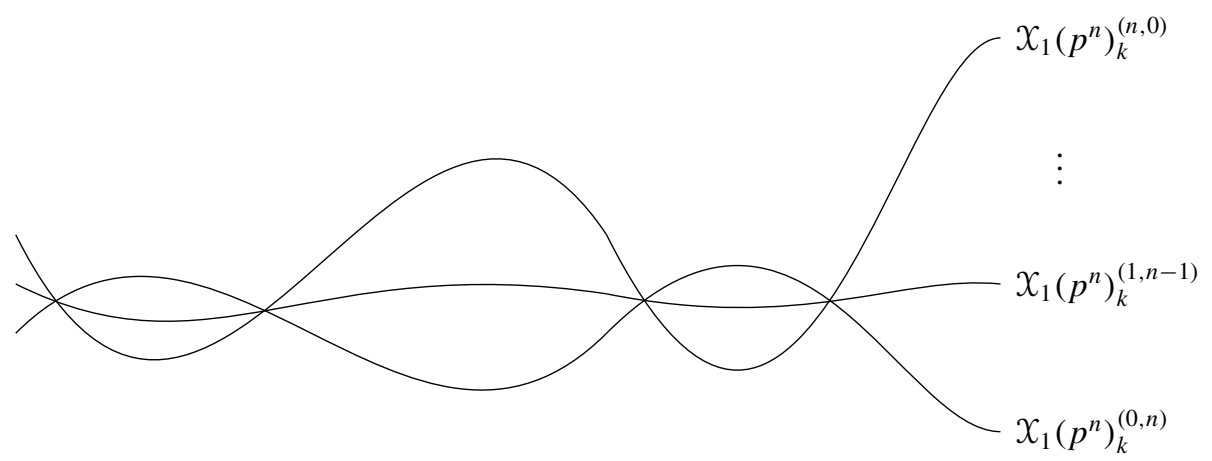

$X_{1}\left(p^{n}\right)$ over a perfect field $k$ of characteristic $p$

Definition 2.14. Let $S \in \mathrm{Sch} / \mathbb{F}_{p}$, and let $H \leq\left(\mathbb{Z} /\left(p^{n}\right)\right)^{2}$ such that $H \cong \mathbb{Z} /\left(p^{n}\right)$ (hence also $\left.\left(\mathbb{Z} /\left(p^{n}\right)\right)^{2} / H \cong \mathbb{Z} /\left(p^{n}\right)\right)$. Let $E / S$ be a generalized elliptic curve. Let $(P, Q)$ be a $\left[\Gamma\left(p^{n}\right)\right]$-structure on $E$, corresponding to a group homomorphism $\phi:\left(\mathbb{Z} /\left(p^{n}\right)\right)^{2} \rightarrow E\left[p^{n}\right],(1,0) \mapsto P,(0,1) \mapsto Q$. We say that $(P, Q)$ has component label $H$ if

- $\phi(H) \subseteq \operatorname{ker}\left(F^{n}\right)$, where $F: E \rightarrow E^{(p)}$ is the relative Frobenius, and

- the resulting group scheme homomorphism $\mathbb{Z} /\left(p^{n}\right) \cong\left(\mathbb{Z} /\left(p^{n}\right)\right)^{2} / H \rightarrow E^{\left(p^{n}\right)}=$ $E / \operatorname{ker}\left(F^{n}\right)$ is a $\left[\Gamma_{1}\left(p^{n}\right)\right]$-structure on $E^{\left(p^{n}\right)}$. (This is independent of the choice of isomorphism $\left(\mathbb{Z} /\left(p^{n}\right)\right)^{2} / H \cong \mathbb{Z} /\left(p^{n}\right)$, although the $\left[\Gamma_{1}\left(p^{n}\right)\right]$-structure obtained depends on this choice.)

We define $\mathcal{Y}\left(p^{n}\right)_{S}^{H}\left(\right.$ resp. $\left.X\left(p^{n}\right)_{S}^{H}\right)$ to be the substack of $y\left(p^{n}\right)_{S}\left(\operatorname{resp} . X\left(p^{n}\right)_{S}\right)$ associating to a scheme $T / S$ the groupoid of tuples $(E,(P, Q))$, where $E / T$ is an 
elliptic curve (resp. generalized elliptic curve) and $(P, Q)$ is a $\left[\Gamma\left(p^{n}\right)\right]$-structure on $E$ of component label $H$.

Theorem 2.15 [Katz and Mazur 1985, 13.7.6]. Let $k$ be a perfect field of characteristic p. $\mathcal{Y}\left(p^{n}\right)_{k}$ (resp. $\left.X\left(p^{n}\right)_{k}\right)$ is the disjoint union, with crossings at the supersingular points, of the substacks $y\left(p^{n}\right)_{k}^{H}\left(\right.$ resp. $\left.X\left(p^{n}\right)_{k}^{H}\right)$ for $H \leq\left(\mathbb{Z} /\left(p^{n}\right)\right)^{2}$ with $H \cong \mathbb{Z} /\left(p^{n}\right)$.

The proof of the above theorem immediately generalizes to a slightly more general setting which we will find useful when studying compactified stacks of $[\Gamma(N)]$-structures below. Let $K \leq\left(\mathbb{Z} /\left(p^{n}\right)\right)^{2}$, and write $G_{K}=\left(\mathbb{Z} /\left(p^{n}\right)\right)^{2} / K$. Then there exist integers $m \geq l \geq 0$ with $G_{K} \cong \mathbb{Z} /\left(p^{m}\right) \times \mathbb{Z} /\left(p^{l}\right)$. Suppose that $l \geq 1$, so $G_{K} \cong \mathbb{Z} /\left(p^{m}\right) \times \mathbb{Z} /\left(p^{l}\right)$ with $m \geq l \geq 1$. Given a $G_{K}$-structure $\phi$ on an ordinary elliptic curve $E / T / \mathbb{F}_{p}$ (in the sense of Definition 2.5), étale locally on $T$ we can consider the composite

$$
G_{K}=\mathbb{Z} /\left(p^{m}\right) \times \mathbb{Z} /\left(p^{l}\right) \stackrel{\phi}{\rightarrow} E\left[p^{n}\right] \cong \mathbb{Z} /\left(p^{n}\right) \times \mu_{p^{n}} \stackrel{\pi_{1}}{\rightarrow} \mathbb{Z} /\left(p^{n}\right) .
$$

Since $\phi$ is a $G_{K}$-structure, the kernel and image of this composite are necessarily cyclic. The same argument used in [Katz and Mazur 1985, 13.7.6] to prove the above theorem shows that in characteristic $p, y_{K}$ breaks up into a union of substacks indexed in this way by the possible kernels of group homomorphisms $G_{K} \rightarrow \mathbb{Z} /\left(p^{n}\right)$, subject to the condition on the image just described. So we can describe $y_{K}$ as a union of closed substacks indexed by the set

$$
L_{K}:=\left\{H \leq G_{K} \mid H \text { and } G / H \text { are both cyclic }\right\} .
$$

Since $G_{K} \cong \mathbb{Z} /\left(p^{m}\right) \times \mathbb{Z} /\left(p^{l}\right)$ with $m \geq l \geq 1$, we have that $H \in L_{K}$ if and only if $H \cong \mathbb{Z} /\left(p^{m}\right)$ or $H \cong \mathbb{Z} /\left(p^{l}\right)$.

The rigorous definition, accounting for elliptic curves that might not be ordinary and for the case where $G_{K}$ is cyclic, is as follows:

Definition 2.16. Let $K \leq(\mathbb{Z} /(N))^{2}$ with corresponding quotient $G_{K}=(\mathbb{Z} /(N))^{2} / K$. We write $y_{K}\left(\right.$ resp. $\left.X_{K}\right)$ for the algebraic stack over $\operatorname{Spec}(\mathbb{Z})$ associating to a scheme $S$ the groupoid of pairs $(E, \phi)$, where $E / S$ is an elliptic curve (resp. generalized elliptic curve) and $\phi$ is a $G_{K}$-structure on $E$.

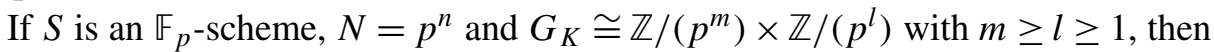
for any $H \in L_{K}$, we define $y_{K, S}^{H} \subset y_{K, S}$ (resp. $x_{K, S}^{H} \subset X_{K, S}$ ) to be the substack associating to a scheme $T / S$ the groupoid of pairs $(E, \phi)$, where $E / T$ is an elliptic curve (resp. generalized elliptic curve) and $\phi: G_{K} \rightarrow E\left[p^{m}\right]$ is a $G_{K}$-structure such that:

- $\phi(H) \subset \operatorname{ker}\left(F^{m}\right)$, where $F^{m}: E \rightarrow E^{\left(p^{m}\right)}$ is the $m$-fold relative Frobenius on $E$.

- The resulting group scheme homomorphism $G_{K} / H \rightarrow E / \operatorname{ker}\left(F^{m}\right) \cong E^{\left(p^{m}\right)}$ is a $G_{K} / H$-structure on $E^{\left(p^{m}\right)}$ in the sense of [Katz and Mazur 1985, §1.5]. 
In this case we say that the $G_{K}$-structure $\phi$ has component label $H$.

If $G_{K} \cong \mathbb{Z} /\left(p^{m}\right)$ (that is, $\left.l=0\right)$, the stack $y_{K}$ (resp. $\left.X_{K}\right)$ is isomorphic to $y_{1}\left(p^{m}\right)$ (resp. $\left.X_{1}\left(p^{m}\right)\right)$, and for $H \cong \mathbb{Z} /\left(p^{a}\right) \in L_{K}$ and $S \in \operatorname{Sch} / \mathbb{F}_{p}$ we define $y_{K, S}^{H}$ (resp. $x_{K, S}^{H}$ ) to be the substack $y_{1}\left(p^{m}\right)_{S}^{(a, m-a)} \subset y_{1}\left(p^{m}\right)_{S}\left(\right.$ resp. $x_{1}\left(p^{m}\right)_{S}^{(a, m-a)} \subset$ $\left.X_{1}\left(p^{m}\right)_{S}\right)$, as in Definition 2.10. We still say $y_{K, S}^{H}$ and $X_{K, S}^{H}$ classify $G_{K}$-structures of component label $H$.

The result is:

Theorem 2.17. Let $k$ be a perfect field of characteristic p. $y_{K, k}\left(\right.$ resp. $\left.x_{K, k}\right)$ is the disjoint union, with crossings at the supersingular points, of the closed substacks $y_{K, k}^{H}\left(\right.$ resp. $\left.X_{K, k}^{H}\right)$ for $H \in L_{K}$.

We also note that an analogue of Lemma 2.11 holds in this case, which we record for future use. It is clear that if $K^{\prime} \leq K \leq\left(\mathbb{Z} /\left(p^{n}\right)\right)^{2}$, giving a canonical quotient map $\pi: G_{K^{\prime}} \rightarrow G_{K}$, and if $\phi: G_{K} \rightarrow E^{\mathrm{sm}}$ is a $G_{K}$-structure on a generalized elliptic curve $E / S / \mathbb{F}_{p}$, then a necessary condition for the composite $\phi \circ \pi: G_{K^{\prime}} \rightarrow E^{\mathrm{sm}}$ to be a $G_{K^{\prime}}$-structure is that $\operatorname{ker}(\pi)$ is cyclic. In fact, unwinding the definitions we immediately deduce:

Lemma 2.18. Let $E / S / \mathbb{F}_{p}$ be a generalized elliptic curve with no supersingular fibers. Let $K^{\prime} \leq K \leq\left(\mathbb{Z} /\left(p^{n}\right)\right)^{2}$ such that the canonical quotient $\pi: G_{K^{\prime}} \rightarrow G_{K}$ has cyclic kernel. Let $H \in L_{K}$ and suppose that $\phi: G_{K} \rightarrow E$ is a $G_{K}$-structure with component label $H$. Then $\phi \circ \pi$ is a $G_{K^{\prime}}$ structure on $E$ if and only if $\pi^{-1}(H) \subseteq G_{K^{\prime}}$ is cyclic, in which case $\phi \circ \pi$ has component label $\pi^{-1}(H) \in L_{K^{\prime}}$.

\section{Generalities/review of twisted stable maps}

Twisted stable maps. We will be studying moduli stacks of elliptic curves embedded in moduli stacks of twisted stable maps to tame stacks. We now recall the relevant definitions and results relating to twisted stable maps.

Definition 3.1 [Abramovich et al. 2008b, 2.2]. Let $G$ be a group scheme over a scheme $S$. Write $\mathrm{QCoh}^{G}(S)$ for the category of $G$-equivariant quasicoherent sheaves on $S$; writing $\mathscr{B} G$ for the classifying stack of $G$ over $S$, this is equivalent to $\mathrm{QCoh}(\mathscr{B} G)$ [Abramovich et al. 2008b, §2.1]. We say that $G$ is linearly reductive if the functor $\operatorname{QCoh}^{G}(S) \rightarrow \mathrm{QCoh}(S), \mathscr{F} \mapsto \mathscr{F}^{G}$ is exact, or equivalently if the pushforward $\mathrm{QCoh}(\mathscr{B} G) \rightarrow \mathrm{QCoh}(S)$ is exact.

Linearly reductive group schemes are classified in [Abramovich et al. 2008b, §2.3]. The examples in which we are most interested for this paper are the finite flat commutative linearly reductive group schemes $\mu_{N}$ and $\mu_{N}^{2}$ over $S$.

Definition 3.2 [Abramovich et al. 2008b, 3.1]. Let $\mathscr{X}$ be a locally finitely presented algebraic stack over a scheme $S$, with finite inertia. By [Keel and Mori 1997], $\mathscr{X}$ 
has a coarse moduli space $\rho: \mathscr{X} \rightarrow X$, with $\rho$ proper. We say that $\mathscr{X} / S$ is tame if $\rho_{*}: \mathrm{QCoh}(\mathscr{X}) \rightarrow \mathrm{QCoh}(X)$ is exact.

As observed in [Abramovich et al. 2008b, §3], for any finite flat group scheme $G$ over a scheme $S$, the classifying stack $\mathscr{B} G$ over $S$ is tame if and only if $G$ is linearly reductive. So in particular the classifying stacks $\mathscr{B} \mu_{N}$ and $\mathscr{B} \mu_{N}^{2}$ are always tame.

Definition 3.3 [Abramovich et al. 2011, §2]. An n-marked twisted curve over a scheme $S$ is a proper tame stack $\mathscr{C}$ over $S$, with connected dimension-1 geometric fibers, and coarse space $f: C \rightarrow S$ a nodal curve over $S$; together with $n$ closed substacks

$$
\left\{\Sigma_{i} \subset \mathscr{C}\right\}_{i=1}^{n}
$$

which are fppf gerbes over $S$ mapping to $n$ markings $\left\{p_{i} \in C^{\mathrm{sm}}(S)\right\}$, such that:

- the preimage in $\mathscr{b}$ of the complement $C^{\prime} \subset C$ of the markings and singular locus of $C / S$ maps isomorphically onto $C^{\prime}$,

- if $\bar{p} \rightarrow C$ is a geometric point mapping to the image in $C$ of a marking $\Sigma_{i} \subset \mathscr{C}$, then

$$
\operatorname{Spec}\left(\mathscr{O}_{C, \bar{p}}\right) \times_{C} \mathscr{b} \simeq\left[D^{\mathrm{sh}} / \mu_{r}\right]
$$

for some $r \geq 1$, where $D^{\text {sh }}$ is the strict Henselization at $\left(\mathfrak{m}_{S, f(\bar{p})}, z\right)$ of

$$
D=\operatorname{Spec}\left(O_{S, f(\bar{p})}[z]\right)
$$

and $\zeta \in \mu_{r}$ acts by $z \mapsto \zeta \cdot z$, and

- if $\bar{p} \rightarrow C$ is a geometric point mapping to a node of $C$, then

$$
\operatorname{Spec}\left(\mathscr{O}_{C, \bar{p}}\right) \times_{C} \mathscr{b} \simeq\left[D^{\mathrm{sh}} / \mu_{r}\right]
$$

for some $r \geq 1$, where $D^{\text {sh }}$ is the strict Henselization at $\left(\mathfrak{m}_{S, f(\bar{p})}, x, y\right)$ of

$$
D=\operatorname{Spec}\left(\mathbb{O}_{S, f(\bar{p})}[x, y] /(x y-t)\right)
$$

for some $t \in \mathfrak{m}_{S, f(\bar{p})}$, and $\zeta \in \mu_{r}$ acts by $x \mapsto \zeta \cdot x$ and $y \mapsto \zeta^{-1} \cdot y$.

We say a twisted curve $\mathscr{C} / S$ has genus $g$ if the geometric fibers of its coarse space $C / S$ have arithmetic genus $g$, and we say an $n$-marked genus- $g$ twisted curve $\mathscr{b} / S$ is stable if the genus- $g$ curve $C / S$ with the markings $\left\{p_{i}\right\}$ is an $n$-marked genus- $g$ stable curve over $S$.

Example 3.4. Over any base scheme $S$, consider a Néron 1-gon $C / S$ as in Section 2. We have $C^{\mathrm{sm}} \cong \mathbb{G}_{m}$, and $C$ admits the structure of a generalized elliptic curve with an action $C^{\mathrm{sm}} \times C \rightarrow C$ extending the group scheme structure of $\mathbb{G}_{m}$. For any positive integer $N$, the inclusion $\mu_{N} \subset \mathbb{G}_{m}$ determines an action of $\mu_{N}$ on $C$, and the stack quotient $\mathscr{C}:=\left[C / \mu_{N}\right]$ is a twisted stable curve over $S$, with coarse space 
$f: C^{\prime} \rightarrow S$ again a Néron 1-gon. If $\bar{p} \rightarrow C^{\prime}$ is a geometric point mapping to a node of $C^{\prime}$, then

$$
\operatorname{Spec}\left(\mathscr{O}_{C^{\prime}, \bar{p}}\right) \times_{C^{\prime}} \mathscr{C} \simeq\left[D^{\mathrm{sh}} / \mu_{N}\right],
$$

where $D^{\text {sh }}$ denotes the strict Henselization of

$$
D:=\operatorname{Spec}\left(\mathcal{O}_{S, f(\bar{p})}[x, y] /(x y)\right)
$$

at the point $\left(\mathfrak{m}_{S, f(\bar{p})}, x, y\right)$ and $\zeta \in \mu_{N}$ acts by $x \mapsto \zeta \cdot x$ and $y \mapsto \zeta^{-1} \cdot y$. We will refer to this twisted curve as the standard $\mu_{N}$-stacky Néron 1-gon over $S$.

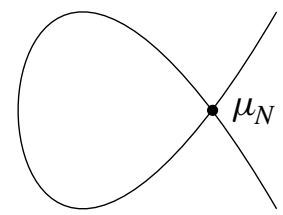

Standard $\mu_{N}$-stacky Néron 1-gon

Definition 3.5 [Abramovich et al. 2011, §4]. Let $\mathscr{X}$ be a finitely presented algebraic stack, proper over a scheme $S$ and with finite inertia. A twisted stable map to $\mathscr{X}$ from an $n$-marked twisted curve $\left(\mathscr{C} / S,\left\{\Sigma_{i}\right\}\right)$ over $S$ with coarse space $\left(C / S,\left\{p_{i}\right\}\right)$ is a morphism $\mathscr{C} \rightarrow \mathscr{X}$ of stacks over $S$ such that.

- $\mathscr{C} \rightarrow \mathscr{X}$ is a representable morphism.

- The induced map $C \rightarrow X$ is a stable map from $\left(C,\left\{p_{i}\right\}\right)$ to $X$.

Over any base scheme $S$, we write $\mathscr{K}_{g, n}(\mathscr{X})$ for the stack over $S$ associating to a scheme $T / S$ the groupoid of pairs $(\mathscr{b}, \alpha)$, where $\mathscr{b} / T$ is an $n$-marked twisted curve whose coarse space $C / T$ is a genus- $g$ nodal curve, and $\alpha: \mathscr{C} \rightarrow \mathscr{X} \times{ }_{S} T$ is a twisted stable map.

Proposition 3.6 [Abramovich et al. 2011, 4.2]. For $\mathscr{L}$ as above, $\mathscr{K}_{g, n}(\mathscr{X})$ is a locally finitely presented algebraic stack over $S$.

There is a natural morphism of stacks $\mathscr{K}_{g, n}(\mathscr{X}) \rightarrow \bar{M}_{g, n}(X)$ (where $\bar{M}_{g, n}(X)$ is the usual Kontsevich stack of stable maps into $X$ ) defined by passing to coarse spaces. In particular, note that since a twisted stable map is required to be representable, if $\mathscr{X}$ is representable then this map is an equality.

Theorem 3.7 [Abramovich et al. 2011, 4.3]. Let $\mathscr{X}$ be as above, and also assume that $\mathscr{X}$ is tame. Then $\mathscr{K}_{g, n}(\mathscr{X})$ is proper and quasifinite over $\bar{M}_{g, n}(X)$.

A word of caution: the twisted stable maps of [Abramovich et al. 2011] are a generalization of what are referred to as balanced twisted stable maps in [Abramovich and Vistoli 2002; Abramovich et al. 2003]; we are only interested in balanced maps for this paper, so hopefully our notation $\mathscr{K}_{g, n}(\mathscr{X})$ will not cause any confusion. 
Let $\left(\mathscr{C} / S,\left\{\Sigma_{i}\right\}\right)$ be an $n$-marked twisted curve with coarse space $C / S$. If $\bar{p} \rightarrow C$ is a geometric point mapping to the image in $C$ of the gerbe $\Sigma_{i}$, such that $\operatorname{Spec}\left(\mathscr{O}_{C}, \bar{p}\right) \times{ }_{C} \mathscr{C} \simeq\left[D^{\text {sh }} / \mu_{r}\right]$ as in Definition 3.3, then the integer $r$ is uniquely determined by $\bar{p}$, and we call $r$ the local index of $\mathscr{b}$ at $\bar{p}$. In fact one verifies immediately (see [Abramovich and Vistoli 2002, 8.5.1]) that the local index only depends on $i$ and on the image of $\bar{p}$ in $S$, and that the function $\epsilon_{i}: S \rightarrow \mathbb{Z}_{>0}$ sending $s=f(\bar{p}) \in S$ to the local index $r$ is locally constant.

Notation 3.8. Let $\boldsymbol{e}=\left(e_{1}, \ldots, e_{n}\right) \in \mathbb{Z}_{>0}^{n}$. We say an $n$-marked twisted curve $\left(\mathscr{C} / S,\left\{\Sigma_{i}\right\}\right)$ has global index $\boldsymbol{e}$ if $\epsilon_{i}$ is constant of value $e_{i}$ for all $i=1, \ldots, n$.

If $\mathscr{X} / S$ is a finitely presented algebraic stack, proper over $S$ and with finite inertia, we write $\mathscr{K}_{g, n}^{e}(\mathscr{X})$ for the substack of $\mathscr{K}_{g, n}(\mathscr{X})$ associating to a scheme $T / S$ the groupoid of pairs $(\mathscr{C}, \alpha)$, where $\mathscr{C} / T$ is an $n$-marked genus- $g$ twisted curve of global index $\boldsymbol{e}$, and $\alpha: \mathscr{C} \rightarrow \mathscr{X} \times{ }_{S} T$ is a twisted stable map. Since the functions $\epsilon_{i}: S \rightarrow \mathbb{Z}_{>0}$ are locally constant, we see that $\mathscr{K}_{g, n}^{e}(\mathscr{X})$ is an open and closed substack of $\mathscr{K}_{g, n}(\mathscr{X})$, and

$$
\mathcal{K}_{g, n}(\mathscr{X})=\coprod_{\boldsymbol{e} \in \mathbb{Z}_{>0}^{n}} \mathscr{K}_{g, n}^{\boldsymbol{e}}(\mathscr{X}) .
$$

In this paper we will generally only be interested in the case $\boldsymbol{e}=(1, \ldots, 1)$, in which case $\mathscr{K}_{g, n}^{e}(\mathscr{C})$ classifies twisted stable maps to $\mathscr{X}$ from twisted curves whose markings are honest sections. We will write $\mathscr{K}_{g, n}^{\prime}(\mathscr{X})$ for $\mathscr{K}_{g, n}^{(1, \ldots, 1)}(\mathscr{X})$.

Notation 3.9. We write $\mathscr{K}_{g, n}^{\circ}(\mathscr{X})$ for the open substack of $\mathscr{K}_{g, n}^{\prime}(\mathscr{X})$ associating to $T / S$ the groupoid of pairs $(\mathscr{C}, \alpha)$, where $\mathscr{C} / T$ is a smooth $n$-marked genus- $g$ curve (with no stacky structure) and $\alpha: \mathscr{C} \rightarrow \mathscr{X} \times{ }_{S} T$ is a twisted stable map.

Twisted covers and Picard schemes of twisted curves. If $G / S$ is a linearly reductive finite flat group scheme, then we have already observed that $\mathscr{B} G$ is tame. So in this case we can consider the algebraic stack $\mathscr{K}_{g, n}(\mathscr{B} G)$, which is proper and quasifinite over the Deligne-Mumford stack $\bar{M}_{g, n}$ by Theorem 3.7, since the coarse space of $\mathscr{B} G$ is $S$.

Theorem 3.10 [Abramovich et al. 2011, 5.1]. Let $G / S$ be a linearly reductive finite flat group scheme. Then $\mathscr{K}_{g, n}(\mathscr{B} G)$ is flat over $S$, with local complete intersection fibers.

Definition 3.11. Since a map $\mathscr{C} \rightarrow \mathscr{B} G$ is equivalent to a $G$-torsor $P \rightarrow \mathscr{C}$, it is often convenient to view $\mathscr{K}_{g, n}(\mathscr{B} G)$ as classifying such torsors. A $G$-torsor $P \rightarrow \mathscr{C}$ is a twisted $G$-cover of $\mathscr{C}$ if it arises in this way.

Note that since the coarse space of $\mathscr{B} G$ is $S$, the condition that the map $\mathscr{b} \rightarrow \mathscr{B} G$ is stable just says that $C / S$ is stable in the usual sense. So for $G$ as above, $\mathscr{K}_{g, n}(\mathscr{B} G)$ 
can and will be viewed as the stack classifying twisted $G$-covers of $n$-marked genus- $g$ twisted stable curves.

Still writing $G$ for a finite flat linearly reductive group scheme over $S$, suppose in addition that $G$ is commutative. Then every object of $\mathscr{K}_{g, n}(\mathscr{B} G)$ canonically contains $G$ in the center of its automorphism group scheme (determining a unique subgroup stack $H$ in the center of the inertia stack of $\mathscr{K}_{g, n}(\mathscr{B} G)$ such that for any object $\xi \in \mathscr{K}_{g, n}(\mathscr{B} G)(T)$ the pullback of $H$ to $T$ is $\left.G\right)$. We can therefore apply the rigidification procedure described in [Abramovich et al. 2003, §5.1] and generalized in [Abramovich et al. 2008b, Appendix A], thereby "removing" $G$ from the automorphism group of each object:

Definition 3.12. $\overline{\mathscr{K}}_{g, n}(\mathscr{B} G)$ is the rigidification of $\mathscr{K}_{g, n}(\mathscr{B} G)$ with respect to the copy of $G$ naturally contained in the center of its inertia stack. We refer to the objects of $\overline{\mathscr{K}}_{g, n}(\mathscr{B} G)$ as rigidified twisted stable maps into $\mathscr{B} G$, or rigidified twisted $G$-covers of $n$-marked genus- $g$ twisted stable curves. We write $\overline{\mathscr{K}}_{g, n}^{\circ}(\mathscr{B} G)$ for the open substack corresponding to smooth curves and $\overline{\mathscr{K}}_{g, n}^{\prime}(\mathscr{B} G)$ for the open and closed substack corresponding to twisted stable curves whose markings are representable.

In [Abramovich et al. 2008a, §C.2] it is shown that the rigidification of an algebraic stack with respect to a group scheme admits a natural moduli interpretation. In this paper we are only interested in the case where $G$ is diagonalizable, in which case $\overline{\mathscr{K}}_{g, n}(\mathscr{B} G)$ can be given a more concrete moduli interpretation which we will now describe.

Given a twisted curve $\mathscr{C}$ over a scheme $S$, let $\mathcal{P} i c_{\mathscr{C} / S}$ denote the stack associating to $T / S$ the groupoid of line bundles on $\mathscr{C} \times{ }_{S} T$.

Proposition 3.13 [Abramovich et al. 2011, 2.7]. $\mathcal{P i c}_{\mathscr{C} / S}$ is a smooth locally finitely presented algebraic stack over $S$, and for any $\mathscr{L} \in \mathcal{P}_{c_{\mathscr{C} / S}}(T)$ the group scheme $\operatorname{Aut}_{T}(\mathscr{L})$ is canonically isomorphic to $\mathbb{G}_{m, T}$.

Write $\operatorname{Pic}_{\mathscr{C} / S}$ for the rigidification of this stack with respect to $\mathbb{G}_{m} ; \mathrm{Pic}_{\mathscr{C} / S}$ is none other than the relative Picard functor of $\mathscr{C} / S$. From the analysis of $\operatorname{Pic}_{\mathscr{C}} / S$ in [Abramovich et al. 2011, §2] we have:

Proposition 3.14. $\mathrm{Pic}_{\mathscr{C} / S}$ is a smooth group scheme over $S$, and if $\pi: \mathscr{b} \rightarrow C$ is the coarse space of $\mathscr{b} / S$, then there is a short exact sequence of group schemes over $S$

$$
0 \rightarrow \operatorname{Pic}_{C / S} \stackrel{\pi^{*}}{\rightarrow} \operatorname{Pic}_{\mathscr{C} / S} \rightarrow W \rightarrow 0
$$

with $W$ quasifinite and étale over $S$. 
In fact, since $\pi_{*} \mathbb{G}_{m}=\mathbb{G}_{m}$ we can deduce from the exact sequence of low-degree terms of the fppf Leray spectral sequence

$$
E_{2}^{p, q}=H^{p}\left(C, \boldsymbol{R}^{q} \pi_{*} \mathbb{G}_{m}\right) \Rightarrow H^{p+q}\left(\mathscr{C}, \mathbb{G}_{m}\right)
$$

that $W$ is the sheaf associated to the presheaf $T \mapsto H^{0}\left(C_{T}, \boldsymbol{R}^{1} \pi_{*} \mathbb{G}_{m}\right)$ (where we still write $\pi: \mathscr{C}_{T} \rightarrow C_{T}$ for the morphism induced by base change from $\pi: \mathscr{C} \rightarrow C$ ).

For any integer $N$ annihilating $W$, we get a natural morphism

$$
\times N: \mathrm{Pic}_{\mathscr{C} / S} \rightarrow \mathrm{Pic}_{C / S} .
$$

Definition 3.15 [Abramovich et al. 2011, 2.11]. The generalized Jacobian of $\mathscr{b}$ is

$$
\operatorname{Pic}_{\mathscr{C} / S}^{0}:=\operatorname{Pic}_{\mathscr{C} / S} \times \times N, \mathrm{Pic}_{C / S} \operatorname{Pic}_{C / S}^{0},
$$

where $\mathrm{Pic}_{C / S}^{0}$ is the fiberwise connected component of the identity in the group scheme $\mathrm{Pic}_{C / S}$.

$\operatorname{Pic}_{\mathscr{C} / S}^{0}$ is independent of $N$ and is viewed as classifying line bundles of fiberwise degree 0 on $\mathscr{b} / S$.

Remark 3.16. Unlike the case of $\operatorname{Pic}_{C / S}^{0}$ for $C / S$ a (nonstacky) genus- $g$ curve, the geometric fibers of $\operatorname{Pic}_{\mathscr{C} / S}^{0}$ need not be connected for $\mathscr{C} / S$ a twisted curve. In fact, when $\mathscr{C} / S$ is a 1-marked genus-1 twisted stable curve, $\mathrm{Pic}_{\mathscr{C} / S}^{0}$ behaves like the smooth part of a generalized elliptic curve over $S$. For instance, if $\mathscr{C}=\left[C / \mu_{N}\right]$ for $C / S$ a Néron 1-gon as in Example 3.4, it is easily verified that $\operatorname{Pic}_{\mathscr{C} / S}^{0} \cong \mathbb{G}_{m} \times \mathbb{Z} /(N)$. Standard $\mu_{N}$-stacky Néron 1-gons will play an analogous role in this paper to that of Néron polygons in [Deligne and Rapoport 1973; Conrad 2007]. In particular, we have:

Lemma 3.17. Let $k$ be an algebraically closed field, and $\mathscr{C} / k$ a 1-marked genus1 twisted stable curve, with no stacky structure at its marking, such that the coarse space $C / k$ is not smooth. Then $\mathscr{b}$ is a standard $\mu_{N}$-stacky Néron 1-gon (Example 3.4) for some (unique) positive integer $N$.

Proof. It follows from [Deligne and Rapoport 1973, II.1.15] that the 1-marked genus-1 stable curve $C / k$ is a Néron 1-gon. Write $\pi: \mathscr{C} \rightarrow C$ for the map exhibiting $C$ as the coarse space of $\mathscr{C}$. Write $\widetilde{C}=\mathbb{P}^{1} \rightarrow C$ for the normalization of $C$, and write $\widetilde{\mathscr{C}}$ for the following fiber product:

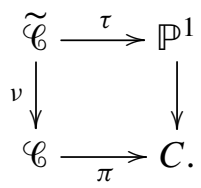

We have a short exact sequence of fppf sheaves on $\mathbb{P}^{1}$

$$
0 \rightarrow \mathbb{O}_{\mathbb{P} 1}^{\times} \rightarrow \tau_{*} \mathbb{O}_{\widetilde{\mathscr{C}}}^{\times} \rightarrow \mathscr{F} \rightarrow 0,
$$


where $\mathscr{F}$ is the pushforward to $\mathbb{P}^{1}$ of $\mathbb{O}_{Z}^{\times}$, for $Z \cong \mathscr{B} \mu_{N} \sqcup \mathscr{B} \mu_{N}$ the preimage in $\widetilde{\mathscr{C}}$ of the (stacky) node of $\mathscr{C}$. This induces an exact sequence

$$
0 \rightarrow \operatorname{Pic}\left(\mathbb{P}^{1}\right) \rightarrow \operatorname{Pic}(\widetilde{\mathscr{C}}) \rightarrow \operatorname{Pic}(Z) \rightarrow H^{2}\left(\mathbb{P}^{1}, \mathbb{O}_{\mathbb{P}^{1}}^{\times}\right)=0,
$$

inducing an isomorphism $\operatorname{Pic}_{\tilde{\mathscr{C}} / k}^{0} \cong \operatorname{Pic}(Z) \cong \mathbb{Z} /(N) \times \mathbb{Z} /(N)$ since $\operatorname{Pic}_{\mathbb{P}^{1} / k}^{0}=0$.

Now consider the short exact sequence of fppf sheaves on $\mathscr{C}$

$$
0 \rightarrow \mathrm{O}_{\mathscr{C}}^{\times} \rightarrow v_{*} \mathrm{O}_{\widetilde{\mathscr{C}}}^{\times} \rightarrow \mathscr{G} \rightarrow 0,
$$

where $\mathscr{G}$ is the pushforward to $\mathscr{C}$ of $\mathbb{O}_{p}^{\times}$for $p \cong \mathscr{B} \mu_{N}$ the (stacky) node of $\mathscr{C}$. This induces an exact sequence

$$
0 \rightarrow k^{\times} \stackrel{\text { id }}{\rightarrow} k^{\times} \stackrel{0}{\rightarrow} k^{\times} \rightarrow \operatorname{Pic}(\mathscr{C}) \rightarrow \operatorname{Pic}(\widetilde{\mathscr{C}}) \rightarrow \operatorname{Pic}\left(\mathscr{B} \mu_{N}\right) .
$$

We have $\operatorname{Pic}\left(\mathscr{B} \mu_{N}\right) \cong \mathbb{Z} /(N)$ and $\operatorname{Pic}_{\mathscr{C} / k}^{0} \cong \mathbb{Z} /(N) \times \mathbb{Z} /(N)$, and the map

$$
\operatorname{Pic}_{\mathscr{C} / k}^{0} \cong \mathbb{Z} /(N) \times \mathbb{Z} /(N) \rightarrow \mathbb{Z} /(N) \cong \operatorname{Pic}\left(\mathscr{B} \mu_{N}\right)
$$

is given by $(a, b) \mapsto a-b$. In particular the kernel is isomorphic to $\mathbb{Z} /(N)$, so we have a short exact sequence

$$
0 \rightarrow k^{\times} \rightarrow \operatorname{Pic}_{\mathscr{C} / k}^{0} \stackrel{f}{\rightarrow} \mathbb{Z} /(N) \rightarrow 0 .
$$

This sequence splits (noncanonically) since $k^{\times}$is divisible (as $k$ is algebraically closed), so $\operatorname{Pic}_{\mathscr{C} / k}^{0} \cong \mathbb{G}_{m} \times \mathbb{Z} /(N)$.

The fppf exact sequence of sheaves on $\mathscr{b}$

$$
0 \rightarrow \mu_{N} \rightarrow \mathbb{G}_{m} \rightarrow \mathbb{G}_{m} \rightarrow 0
$$

then gives us an isomorphism

$$
\begin{aligned}
H^{1}\left(\mathscr{C}, \mu_{N}\right) & \cong \operatorname{ker}\left(\operatorname{Pic}_{\mathscr{C} / k} \stackrel{\times N}{\longrightarrow} \operatorname{Pic}_{\mathscr{C} / k}\right) \\
& \cong \mu_{N} \times \mathbb{Z} /(N) .
\end{aligned}
$$

Let $C^{\prime} \rightarrow \mathscr{C}$ be the $\mu_{N}$-torsor over $\mathscr{C}$ corresponding to the class

$$
(1,1) \in \mu_{N} \times \mathbb{Z} /(N) \cong H^{1}\left(\mathscr{C}, \mu_{N}\right) .
$$

In view of [Abramovich and Hassett 2011, 2.3.10], $C^{\prime}$ is representable, since $(1,1)$ is the class in $H^{1}\left(\mathscr{C}, \mu_{N}\right)$ of a uniformizing line bundle on $\mathscr{C}$. Let

$$
V=C^{\mathrm{sm}} \cong \mathscr{C} \times{ }_{C} C^{\mathrm{sm}} \stackrel{\mathfrak{l}}{\hookrightarrow} \mathscr{C},
$$

and consider the resulting $\mu_{N}$-torsor $C_{V}^{\prime} \rightarrow V \cong \mathbb{G}_{m}$. The pullback map

$$
\iota^{*}: H^{1}\left(\mathscr{C}, \mu_{N}\right) \cong \mu_{N} \times \mathbb{Z} /(N) \rightarrow \mathbb{Z} /(N) \cong H^{1}\left(\mathbb{G}_{m}, \mu_{N}\right)
$$


is given by $(\zeta, a) \mapsto a$. It therefore follows that $C_{V}^{\prime} \cong \mathbb{G}_{m}$, with the $\mu_{N}$-action given by the standard multiplication action on $\mathbb{G}_{m}$. The quotient map $\mathbb{G}_{m} \rightarrow\left[\mathbb{G}_{m} / \mu_{N}\right]$ $\cong \mathbb{G}_{m}$ is the group scheme homomorphism $x \mapsto x^{N}$.

Fix an étale neighborhood $W$ of the node of $C$ of the form

$$
W=\operatorname{Spec}(k[z, w] /(z w)),
$$

such that

$$
\mathscr{C} \times{ }_{C} W \cong\left[D / \mu_{N}\right]
$$

for $D=\operatorname{Spec}(k[x, y] /(x y))$, with $\zeta \in \mu_{N}$ acting by $x \mapsto \zeta x$ and $y \mapsto \zeta^{-1} y$. The composite

$$
D \rightarrow\left[D / \mu_{N}\right] \rightarrow W
$$

is given by the ring homomorphism $z \mapsto x^{N}, w \mapsto y^{N}$. Since $C^{\prime}$ is representable and $C^{\prime} \times{ }_{C} W \rightarrow \mathscr{C} \times{ }_{C} W$ is a $\mu_{N}$-torsor, it follows that $C^{\prime} \times_{C} W \cong D$ with the above $\mu_{N}$-action. In particular we see that $C^{\prime}$ is a nodal curve with one node.

Composing our original $C^{\prime} \rightarrow \mathscr{C}$ with the coarse space map $\mathscr{b} \rightarrow C$ gives us a finite morphism of nodal curves $C^{\prime} \rightarrow C$, which restricts to the $\mu_{N}$-torsor $\mathbb{G}_{m} \rightarrow \mathbb{G}_{m} \cong C^{\mathrm{sm}}$ and which is totally ramified over the node of $C$. RiemannHurwitz for nodal curves implies that $C^{\prime}$ has arithmetic genus 1 , so $C^{\prime}$ is a Néron 1-gon with smooth locus $C^{\prime \text { sm }}=\mathbb{G}_{m}$. The multiplication action of $\mu_{N}$ on $\mathbb{G}_{m}$ extends uniquely to an action on $C^{\prime}$, and by assumption $\mathscr{C}=\left[C^{\prime} / \mu_{N}\right]$. Thus $\mathscr{C}$ is a standard $\mu_{N}$-stacky 1-gon.

\section{Relationship to moduli of elliptic curves with level structure.}

Notation 3.18. For any finite flat commutative group scheme $G$ over a base scheme $S, \mathcal{H}(G)$ is the stack over $S$ associating to an $S$-scheme $T$ the groupoid of pairs $(E, \phi)$, where $E / T$ is an elliptic curve and $\phi: G^{*} \rightarrow E[N]$ is a homomorphism of group schemes over $T$ (for $G^{*}$ the Cartier dual of $G$ ). For the $S$-scheme $G=\mu_{N}$ we write $\mathcal{H}_{1}(N)$ for $\mathcal{H}\left(\mu_{N}\right)$, and for the $S$-scheme $G=\mu_{N}^{2}$ we write $\mathcal{H}(N)$ for $\mathcal{H}\left(\mu_{N}^{2}\right)$.

As in [Abramovich et al. 2008b, §2.3], we say a finite commutative group scheme is diagonalizable if its Cartier dual is constant, and locally diagonalizable if its Cartier dual is étale.

Lemma 3.19 [Abramovich et al. 2011, 5.7]. Let $G / S$ be a finite diagonalizable commutative group scheme, so $\mathscr{B} G$ is tame (since $G$ is linearly reductive) and $X:=G^{*}$ is constant. For any twisted curve $\mathscr{b} / S$ there is an equivalence of categories between the stack $\operatorname{TORS}_{\mathscr{C} / S}(G)$ classifying $G$-torsors on $\mathscr{C} / S$ and the Picard stack Pic $_{\mathscr{C} / S}[X]$ of morphisms of Picard stacks $X \rightarrow \mathcal{P i c}_{\mathscr{C} / S}$. 
The construction for the above equivalence is as follows: Let $\pi: P \rightarrow \mathscr{C}$ be a $G$-torsor over $\mathscr{C}$. $G$ acts on the sheaf $\pi_{*} \mathcal{O}_{P}$, yielding a decomposition

$$
\pi_{*} \mathscr{O}_{P}=\bigoplus_{\chi \in X} \mathscr{L}_{\chi} .
$$

Each $\mathscr{L}_{\chi}$ is invertible since $P$ is a torsor over $E$, so this determines a morphism of Picard stacks

$$
\phi_{P}: X \rightarrow \mathcal{P i c}_{\mathscr{C} / S}, \quad \chi \mapsto\left[\mathscr{L}_{\chi}\right] .
$$

Conversely, such a morphism $\phi: X \rightarrow \mathcal{P} i c_{\mathscr{C} / S}$ naturally determines an algebra structure on $\bigoplus_{\chi \in X} \phi(\chi)$, giving a $G$-torsor

$$
\underline{\operatorname{Spec}}_{\mathscr{C}}\left(\bigoplus_{\chi \in X} \phi(\chi)\right) \rightarrow \mathscr{C}
$$

with the $G$-action determined by the $X$-grading.

This defines an open immersion from $\mathscr{K}_{g, n}(\mathscr{B} G)$ into the algebraic stack over $S$ associating to an $S$-scheme $T$ the groupoid of pairs $(\mathscr{C}, \phi)$, where $\mathscr{C} / T$ is an $n$-marked genus- $g$ twisted stable curve and $\phi \in \mathcal{P i c}_{\mathscr{C} / T}[X]$. Rigidifying $\mathscr{K}_{g, n}(\mathscr{B} G)$ and $\mathcal{P} i c_{\mathscr{C} / T}$ with respect to the group schemes $G$ and $\mathbb{G}_{m}$, respectively, we have an open immersion from $\overline{\mathscr{K}}_{g, n}(\mathscr{B} G)$ into the stack classifying pairs $(\mathscr{C}, \phi)$, where $\mathscr{C} / T$ is an $n$-marked genus- $g$ twisted stable curve and $\phi: X \rightarrow \operatorname{Pic}_{\mathscr{C} / T}$ is a homomorphism of group schemes over $T$.

Writing $f: \mathscr{C} \rightarrow S$ for the structural morphism, we have Pic $\mathscr{C}_{/ S}=\boldsymbol{R}^{1} f_{*} \mathbb{G}_{m}$. Therefore fppf-locally on $T$, the morphism $\phi$ corresponds to the choice of an $X$ torsor $P \rightarrow \mathscr{C}$, with $P$ representable if and only if $(\mathscr{C}, \phi)$ comes from an object of $\overline{\mathscr{K}}_{g, n}(\mathscr{B} G)$ (refer, for example, to [Abramovich and Hassett 2011, 2.3.10] to see that a morphism from a twisted curve $\mathscr{C}$ to $\mathscr{B} G$ is representable if and only if the corresponding $G$-torsor over $\mathscr{C}$ is representable). This gives us:

Corollary 3.20. For a finite flat diagonalizable group scheme $G / S$, the above construction gives an equivalence between $\overline{\mathscr{K}}_{g, n}\left(\Re_{B} G\right)$ and the stack over $S$ associating to $T / S$ the groupoid of pairs $(\mathscr{b}, \phi)$, where $\mathscr{b} / T$ is an n-marked genus-g twisted stable curve and $\phi: X \rightarrow \operatorname{Pic}_{\mathscr{C} / T}$ is a group scheme homomorphism such that, fppf locally on $T$, the $G$-torsor over $\mathscr{C}$ corresponding to $\phi$ as above is representable.

In particular, we get an equivalence

$$
\overline{\mathscr{K}}_{1,1}^{\circ}(\mathscr{B} G) \simeq \mathscr{H}(G),
$$

since for an elliptic curve $E / S$ every $G$-torsor over $E$ is representable and we canonically have $E \cong \mathrm{Pic}_{E / S}^{0}$. This isomorphism sends $Q \in E(S)$ to the class of the line bundle $\mathscr{L}\left((Q)-\left(0_{E}\right)\right)$, so as a special case we see that if $\phi: \mathbb{Z} /(N) \rightarrow E$ 
is a group scheme homomorphism with $\phi(1)=Q$, the corresponding $\mu_{N}$-torsor over $E$ is of the form

$$
P=\underline{\operatorname{Spec}} E\left(\bigoplus_{a=0}^{N-1} \mathscr{L}\left((a \cdot Q)-\left(0_{E}\right)\right)\right) .
$$

Since $\mathcal{H}_{1}(N)$ naturally contains a closed substack isomorphic to the stack $y_{1}(N)$ over $S$ classifying $\left[\Gamma_{1}(N)\right]$-structures on elliptic curves, we see that the algebraic stack $\overline{\mathscr{K}}_{1,1}\left(\mathscr{B} \mu_{N}\right)$ is a modular compactification of $y_{1}(N)$. Similarly, the algebraic stack $\overline{\mathscr{K}}_{1,1}\left(\mathscr{B} \mu_{N}^{2}\right)$ is a modular compactification of the stack $y(N)$ classifying (not necessarily symplectic) full level- $N$ structures on elliptic curves. The task in both cases is to give a moduli interpretation of the closure of these classical moduli stacks in $\overline{\mathscr{K}}_{1,1}(\mathscr{B} G)$, and we address this in the following sections.

Lemma 3.21. $\overline{\mathscr{K}}_{1,1}^{\circ}\left(\mathscr{B} \mu_{N}\right)$ is dense in $\overline{\mathscr{K}}_{1,1}^{\prime}\left(\mathscr{\Re} \mu_{N}\right)$, and $\overline{\mathscr{K}}_{1,1}^{\circ}\left(\mathscr{\Re} \mu_{N}^{2}\right)$ is dense in $\overline{\mathscr{K}}_{1,1}^{\prime}\left(\mathscr{B} \mu_{N}^{2}\right)$.

Proof. Let $\mathscr{C}_{0}$ be a standard $\mu_{d}$-stacky 1-gon over an algebraically closed field $k$, and let $\phi_{0}: \mathbb{Z} /(N) \rightarrow \mathrm{Pic}_{\mathscr{C}_{k}}^{0} \cong \mathbb{G}_{m} \times \mathbb{Z} /(d)$ be a group scheme homomorphism such that $\left(\mathscr{C}_{0}, \phi_{0}\right) \in \overline{\mathscr{K}}_{1,1}^{\prime}\left(\mathscr{B} \mu_{N}\right)(k)$ (so $d \mid N$ and $\phi_{0}$ meets every component of $\mathrm{Pic}_{\mathscr{C}_{0} / k}^{0}$ ). We need to lift $\left(\mathscr{C}_{0} / k, \phi_{0}\right)$ to a pair $(\mathscr{C}, \phi) \in \overline{\mathscr{K}}_{1,1}^{\prime}\left(\mathscr{B} \mu_{N}\right)(A)$ for a local ring $A$ with residue field $k$, such that the generic fiber of $\mathscr{C}$ is a smooth elliptic curve.

Let $\mathscr{C}_{0}^{\prime} / k$ be a standard $\mu_{N}$-stacky 1-gon. Consider the morphism $\mathscr{C}_{0}^{\prime} \rightarrow \mathscr{B} \mu_{N}$ corresponding to the group scheme homomorphism

$$
\mathbb{Z} /(N) \rightarrow \operatorname{Pic}_{\mathscr{C}_{0}^{\prime} / k}^{0} \cong \mathbb{G}_{m} \times \mathbb{Z} /(N)
$$

sending 1 to $(1, N / d)$. This morphism is not representable; the corresponding $\mu_{N}$-torsor over $\mathscr{C}_{0}^{\prime}$ is as follows:

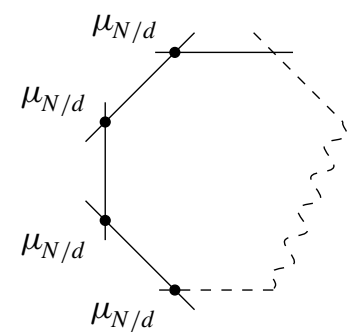

(Stacky $N / d$-gon)

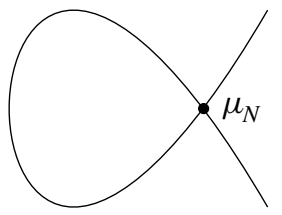

$\mathscr{C}_{0}^{\prime}$

We may factor the morphism $\mathscr{C}_{0}^{\prime} \rightarrow \mathscr{B} \mu_{N}$ as $\mathscr{C}_{0}^{\prime} \rightarrow \mathscr{C}_{0} \rightarrow \mathscr{B} \mu_{N}$, where $\mathscr{C}_{0} \rightarrow \mathscr{B} \mu_{N}$ is the relative coarse moduli space [Abramovich et al. 2011, Theorem 3.1] of $\mathscr{C}_{0}^{\prime} \rightarrow \mathscr{B} \mu_{N}$ : 


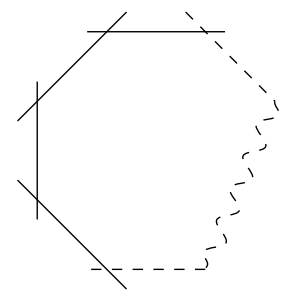

$(N / d$-gon $)$

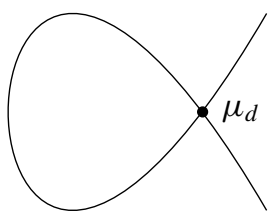

$\mathscr{C}_{0}$

Since this $\mathscr{C}_{0}$ is a standard $\mu_{d}$-stacky 1 -gon, we may identify it with our original twisted curve $\mathscr{C}_{0}$. This gives us a morphism $\mathscr{C}_{0}^{\prime} \rightarrow \mathscr{C}_{0}$, and the resulting pullback map $\mathrm{Pic}_{\mathscr{C}_{0} / k}^{0} \rightarrow \mathrm{Pic}_{\mathscr{C}_{0}^{\prime} / k}^{0}$ is the monomorphism

$$
\mathbb{G}_{m} \times \mathbb{Z} /(d) \rightarrow \mathbb{G}_{m} \times \mathbb{Z} /(N), \quad(\zeta, a) \mapsto\left(\zeta, \frac{N}{d} \cdot a\right) .
$$

Let $\mathscr{E} / k \llbracket q^{1 / N} \rrbracket$ be an $N$-gon Tate curve, so the special fiber of $\mathscr{E}$ is a Néron $N$-gon, $\mathscr{E} \otimes k\left(\left(q^{1 / N}\right)\right)$ is a smooth elliptic curve, and we have an isomorphism $\mathscr{E}^{\mathrm{sm}}[N] \cong \mu_{N} \times \mathbb{Z} /(N)$ of finite flat commutative group schemes over $k \llbracket q^{1 / N} \rrbracket$. Let $Q=(1,1) \in \mathscr{E}^{\mathrm{sm}}[N]$. The relative effective Cartier divisor

$$
D:=\sum_{a \in \mathbb{Z} /(N)}[a \cdot Q]
$$

in $\mathscr{E}$ sm is a subgroup scheme, étale over $k \llbracket q^{1 / N} \rrbracket$, and the quotient $\overline{\mathscr{E}}:=\mathscr{E} / D$ is naturally a generalized elliptic curve whose special fiber is a 1-gon. The stack quotient $\mathscr{C}:=\left[\mathscr{E} / \mathscr{E}^{\mathrm{sm}}[N]\right]$ is naturally a twisted curve, whose generic fiber is an elliptic curve and whose special fiber is $\mathscr{C}_{0}^{\prime}$. Writing $\pi: \overline{\mathscr{E}} \rightarrow \mathscr{C}$ for the natural quotient map, we will see in Section 6 that for any line bundle $\mathscr{L}$ on $\overline{\mathscr{E}}$ there is a canonical decomposition

$$
\pi_{*} \mathscr{L} \cong \bigoplus_{a \in \mathbb{Z} /(N)} \mathscr{L}_{a}
$$

where each $\mathscr{L}_{a}$ is a line bundle on $\mathscr{C}$.

For a section $R \in \mathscr{E}^{\mathscr{s m}}\left(k \llbracket q^{1 / N} \rrbracket\right)$, we write $\bar{R}$ for its image in $\overline{\mathscr{E}} \mathrm{sm}\left(k \llbracket q^{1 / N} \rrbracket\right)$. Then we have the degree-0 line bundle $\mathscr{L}_{R}:=\mathscr{L}\left((\bar{R})-\left(0_{\overline{\mathscr{E}}}\right)\right)$ on $\overline{\mathscr{E}}$, hence a canonical decomposition

$$
\pi_{*} \mathscr{L}_{R} \cong \bigoplus_{a \in \mathbb{Z} /(N)} \mathscr{L}_{R, a} .
$$

We will see in Section 6 that the map $\mathscr{E}^{\mathrm{sm}}[N] \rightarrow \operatorname{Pic}_{\mathscr{C} / k \llbracket q^{1 / N} \rrbracket}^{0}[N]$ sending

$$
R=(\zeta, a) \in \mu_{N} \times \mathbb{Z} /(N) \cong \mathscr{E}^{\mathrm{sm}}[N]
$$

to $\mathscr{L}_{R, a}$ is an isomorphism of group schemes over $k \llbracket q^{1 / N} \rrbracket$. 
Returning to our original pair $\left(\mathscr{C}_{0}, \phi_{0}\right) \in \overline{\mathscr{K}}_{1,1}^{\prime}\left(\mathscr{B} \mu_{N}\right)(k)$, write

$$
\phi(1)=(\zeta, a) \in \mu_{N} \times \mathbb{Z} /(d) \cong \operatorname{Pic}_{\mathscr{C}_{0} / k}^{0}[N] .
$$

Via the map $\mathscr{C}_{0}^{\prime} \rightarrow \mathscr{C}_{0}$ constructed above, this corresponds to $(\zeta,(N / d) \cdot a) \in$ $\mu_{N} \times \mathbb{Z} /(N) \cong \operatorname{Pic}_{\mathscr{C}_{0}^{\prime} / k}^{0}[N] . \mathscr{C}_{0}^{\prime}$ is the special fiber of the twisted curve $\mathscr{C}$, and we have an isomorphism over $k \llbracket q^{1 / N} \rrbracket$

$$
\operatorname{Pic}_{\mathscr{C} / k \llbracket q^{1 / N} \rrbracket}^{0}[N] \cong \mathscr{C}^{\mathrm{sm}}[N] \cong \mu_{N} \times \mathbb{Z} /(N) .
$$

Now $(\zeta,(N / d) \cdot a)$ lifts to a section of $\operatorname{Pic}_{\mathscr{C} / k \llbracket q^{1 / N} \rrbracket}^{0}[N]$, corresponding to a group scheme homomorphism $\mathbb{Z} /(N) \rightarrow \operatorname{Pic}_{\mathscr{b} / k \llbracket q^{1 / N} \rrbracket}^{0}$, hence to a morphism $\mathscr{C} \rightarrow \mathscr{B} \mu_{N}$. Writing $\overline{\mathscr{C}} \rightarrow \mathscr{B} \mu_{N}$ for the relative coarse moduli space and $\phi: \mathbb{Z} /(N) \rightarrow \operatorname{Pic}_{\mathscr{C}}^{0} / k \llbracket q^{1 / N} \rrbracket$ for the corresponding group scheme homomorphism, we see that $\overline{\mathscr{C}} / k \llbracket q^{1 / N} \rrbracket$ is a twisted curve with special fiber $\mathscr{C}_{0}$ and generic fiber an elliptic curve. As desired, $\phi$ extends $\phi_{0}$ and $(\overline{\mathscr{C}}, \phi) \in \overline{\mathscr{K}}_{1,1}^{\prime}\left(\mathscr{B} \mu_{N}\right)\left(k \llbracket q^{1 / N} \rrbracket\right)$.

A similar argument of course applies to $\overline{\mathscr{K}}_{1,1}^{\circ}\left(\mathscr{B} \mu_{N}^{2}\right) \subset \overline{\mathscr{K}}_{1,1}^{\prime}\left(\mathscr{B} \mu_{N}^{2}\right)$.

We will require a concrete description of the $\mu_{N}$-torsor corresponding to a particular sort of $\left[\Gamma_{1}(N)\right]$-structure on an elliptic curve:

Lemma 3.22. Let $K$ be a field and $E / K$ an elliptic curve. Let $Q \in E(K)$ be a $\left[\Gamma_{1}(N)\right]$-structure on $E$ such that the relative effective Cartier divisor

$$
D:=\sum_{a=0}^{N-1}[a \cdot Q]
$$

in $E$ is étale over $\operatorname{Spec}(K)$. Let $P \rightarrow E$ be the $\mu_{N}$-torsor corresponding to $Q$ as in Corollary 3.20:

$$
P=\underline{\operatorname{Spec}}_{E}\left(\bigoplus_{a=0}^{N-1} \mathscr{L}\left((a \cdot Q)-\left(0_{E}\right)\right)\right) .
$$

Then $P$ can be naturally identified with the quotient $E / D$, where $D$ is viewed as a subgroup scheme of $E$, étale of rank $N$ over $\operatorname{Spec}(K)$, with the quotient map $P \rightarrow E$ corresponding to the natural map $E / D \rightarrow E / E[N] \cong E$.

Proof. Consider the $e_{N}$-pairing on $E[N]$, a nondegenerate bilinear pairing of finite flat group schemes over $\operatorname{Spec}(K)$ :

$$
e_{N}: E[N] \times E[N] \rightarrow \mu_{N}
$$

Under our assumptions, the composite map

$$
E[N] / D=\{Q\} \times E[N] / D \hookrightarrow D \times E[N] / D \stackrel{e_{N}}{\rightarrow} \mu_{N}
$$

is an isomorphism of group schemes over $\operatorname{Spec}(K)$. Then via this isomorphism, $\mu_{N}$ acts on the quotient $E / D$ through the natural action of the subgroup scheme 
$E[N] / D \subset(E / D)[N]$, making $E / D$ a $\mu_{N}$-torsor over $E / E[N] \cong E$ with quotient map the obvious one induced from the factorization of $[N]$ as $E \rightarrow E / D \rightarrow$ $E / E[N] \cong E$.

By Lemma 3.19, we may express the $\mu_{N}$-torsor $E / D \rightarrow E$ as

$$
E / D=\underline{\operatorname{Spec}}_{E}\left(\bigoplus_{a=0}^{N-1} \mathscr{L}_{a}\right)
$$

for some line bundles $\mathscr{L}_{a} \in \mathrm{Pic}_{E / K}^{0}[N]$, with the algebra structure determined

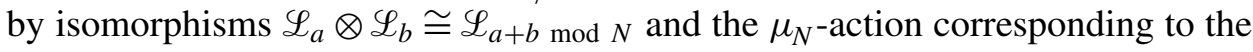
grading. We have a natural isomorphism of group schemes over $K$

$$
\begin{aligned}
E & \rightarrow \operatorname{Pic}_{E / K}^{0} \\
R \in E(K) & \mapsto \mathscr{L}\left((R)-\left(0_{E}\right)\right),
\end{aligned}
$$

so we conclude that $\mathscr{L}_{1} \cong \mathscr{L}\left(\left(Q_{0}\right)-\left(0_{E}\right)\right)$ for some $Q_{0} \in E[N]$, and $\mathscr{L}_{a} \cong \mathscr{L}((a$. $\left.\left.Q_{0}\right)-\left(0_{E}\right)\right)$.

Let $\hat{\pi}: E \rightarrow E / D$ be the isogeny dual to $\pi: E / D \rightarrow E / E[N] \cong E$. Identifying $E \cong \operatorname{Pic}_{E / K}^{0}$ and $E / D \cong \operatorname{Pic}_{(E / D) / K}^{0}, \hat{\pi}$ is simply given by the pullback map $\pi^{*}: \operatorname{Pic}_{E / K}^{0} \rightarrow \operatorname{Pic}_{(E / D) / K}^{0}$. For any line bundle $\mathscr{L}$ on $E / K$ we have

$$
\pi^{*}(\mathscr{L})=\bigoplus_{a=0}^{N-1} \mathscr{L} \otimes \mathscr{L}\left(\left(a \cdot Q_{0}\right)-\left(0_{E}\right)\right),
$$

viewing the direct sum of line bundles on $E$ as a line bundle on

$$
E / D=\underline{\operatorname{Spec}}_{E}\left(\bigoplus \mathscr{L}\left(\left(a \cdot Q_{0}\right)-\left(0_{E}\right)\right)\right)
$$

In particular, for our original $\left[\Gamma_{1}(N)\right]$-structure $Q$,

$$
\pi^{*}\left(\mathscr{L}\left((Q)-\left(0_{E}\right)\right)\right)=\bigoplus_{a=0}^{N-1} \mathscr{L}\left(\left(Q+a \cdot Q_{0}\right)-\left(0_{E}\right)\right) .
$$

But the dual isogeny to $\pi: E / D \rightarrow E / E[N] \cong E$ is the natural quotient map $E \rightarrow$ $E / D$, and this maps $Q$ to $0_{E}$. Therefore the line bundle $\bigoplus \mathscr{L}\left(\left(Q+a \cdot Q_{0}\right)-\left(0_{E}\right)\right)$ on $E / D$ is the trivial line bundle on $E / D$ :

$$
\bigoplus_{a=0}^{N-1} \mathscr{L}\left(\left(Q+a \cdot Q_{0}\right)-\left(0_{E}\right)\right) \cong \bigoplus_{a=0}^{N-1} \mathscr{L}\left(\left(a \cdot Q_{0}\right)-\left(0_{E}\right)\right) .
$$

Therefore $Q$ is contained in the subgroup scheme of $E$ generated by $Q_{0}: Q=b \cdot Q_{0}$ for some $b$. Since $Q_{0} \in E[N]$ and $N$ is the minimal positive integer with $N \cdot Q=0_{E}$, this implies that $b \in(\mathbb{Z} /(N))^{\times}$, and in fact by the definition of the $e_{N}$-pairing we 
have $b=1$, that is, $Q_{0}=Q$. Thus

$$
\underline{\operatorname{Spec}}_{E}\left(\bigoplus_{a=0}^{N-1} \mathscr{L}\left(\left(a \cdot Q_{0}\right)-\left(0_{E}\right)\right)\right)=\underline{\operatorname{Spec}} E\left(\bigoplus_{a=0}^{N-1} \mathscr{L}\left((a \cdot Q)-\left(0_{E}\right)\right)\right)
$$

that is, $E / D \cong P$ with the quotient map $P \rightarrow E$ of the given $\mu_{N}$-action becoming identified with the natural quotient map $E / D \rightarrow E / E[N] \cong E$.

\section{Moduli of elliptic curves in $\overline{\mathscr{K}}_{1,1}\left(\mathscr{\Re}_{N}\right)$}

Reduction mod $\boldsymbol{p}$ of $\mathcal{H}_{1}(N)$. We first describe how the different components of $\overline{\mathscr{K}}_{1,1}^{\circ}\left(\mathscr{B} \mu_{N}\right) \cong \mathcal{H}_{1}(N)$ interact. These results are direct corollaries of [Katz and Mazur 1985, §13.5]. Continue working over an arbitrary base scheme $S$. First consider the case where $N=p^{n}$ is a prime power. We get a closed immersion

$$
\iota^{\left(p^{m}\right)}: y_{1}\left(p^{m}\right) \hookrightarrow \mathcal{H}_{1}\left(p^{n}\right),
$$

for each $0 \leq m \leq n$, sending a pair $(E, \phi) \in y_{1}\left(p^{m}\right)(T)$, where $\phi: \mathbb{Z} /\left(p^{m}\right) \rightarrow$ $E\left[p^{m}\right]$ is a $\left[\Gamma_{1}\left(p^{m}\right)\right]$-structure on $E / T$, to the pair $(E, \widetilde{\phi}) \in \mathcal{H}_{1}\left(p^{n}\right)(T)$, where $\widetilde{\phi}: \mathbb{Z} /\left(p^{n}\right) \rightarrow E\left[p^{n}\right]$ is obtained from $\phi$ by precomposing with the canonical projection $\mathbb{Z} /\left(p^{n}\right) \rightarrow \mathbb{Z} /\left(p^{m}\right)$. These yield a proper surjection of algebraic stacks

$$
y_{1}\left(p^{n}\right) \sqcup y_{1}\left(p^{n-1}\right) \sqcup \cdots \sqcup y_{1}(p) \sqcup y_{1}(1) \rightarrow \mathcal{H}_{1}\left(p^{n}\right)
$$

which is an isomorphism over $S[1 / p]$.

But this is not an isomorphism over $S \otimes \mathbb{F}_{p}$. Recall that by Theorem 2.13, for any perfect field $k$ of characteristic $p, y_{1}\left(p^{m}\right)_{k}$ is the disjoint union, with crossings at the supersingular points, of components

$$
y_{1}\left(p^{m}\right)_{k}^{(m-b, b)} \quad(0 \leq b \leq m)
$$

where an object of $y_{1}\left(p^{m}\right)_{k}^{(m-b, b)}(T)$ is a pair $(E, \phi)$ where $E / T$ is an elliptic curve and $\phi: \mathbb{Z} /\left(p^{m}\right) \rightarrow E\left[p^{m}\right]$ is a $\left[\Gamma_{1}\left(p^{m}\right)\right]-(m-b, b)$-cyclic structure on $E / T$ (Definition 2.10). Such an object corresponds via $\iota^{(m)}$ to the pair $(E, \widetilde{\phi}) \in$ $\mathcal{H}_{1}\left(p^{n}\right)(T)$ as described above.

The key observation is Lemma 2.11: if $\phi: \mathbb{Z} /\left(p^{m}\right) \rightarrow E$ is a $\left[\Gamma_{1}\left(p^{m}\right)\right]-(m-b, b)$ cyclic structure on an ordinary elliptic curve $E / T / \mathbb{F}_{p}$, then

$$
\widetilde{\phi}:=\phi \circ \pi: \mathbb{Z} /\left(p^{n}\right) \rightarrow E
$$

is a $\left[\Gamma_{1}\left(p^{n}\right)\right]-(n-b, b)$-cyclic structure on $E$, where $\pi: \mathbb{Z} /\left(p^{n}\right) \rightarrow \mathbb{Z} /\left(p^{m}\right)$ is the natural projection. Étale locally on $T$ such that $E\left[p^{n}\right] \cong \mu_{p^{n}} \times \mathbb{Z} /\left(p^{n}\right)$ and 
$E\left[p^{m}\right] \cong \mu_{p^{m}} \times \mathbb{Z} /\left(p^{m}\right)$, the $\left[\Gamma_{1}\left(p^{m}\right)\right]-(m-b, b)$-cyclic structure $\phi$ corresponds to a section of

$$
\begin{array}{ll}
\mu_{p^{m}}^{\times} \times\left(\mathbb{Z} /\left(p^{b}\right)\right)^{\times} & \text {if } b<m, \\
\mu_{p^{m}} \times\left(\mathbb{Z} /\left(p^{b}\right)\right)^{\times} & \text {if } b=m,
\end{array}
$$

and the reason that such a section also gives a $\left[\Gamma_{1}\left(p^{n}\right)\right]$-structure is that in characteristic $p$, unlike in characteristic $\neq p$, if $c<n$ and $\mathbb{Z} /\left(p^{c}\right) \rightarrow \mu_{p^{c}}$ is a $\mathbb{Z} /\left(p^{c}\right)$-generator then the composite

$$
\mathbb{Z} /\left(p^{n}\right) \rightarrow \mathbb{Z} /\left(p^{c}\right) \rightarrow \mu_{p^{c}} \hookrightarrow \mu_{p^{n}}
$$

is a $\mathbb{Z} /\left(p^{n}\right)$-generator. This gives us:

Proposition 4.1. Let $k$ be a perfect field of characteristic $p . \mathcal{H}_{1}\left(p^{n}\right)_{k}$ is the disjoint union, with crossings at the supersingular points, of components $Z_{b}$ for $0 \leq b \leq n$, where

$$
Z_{b}=\bigcup_{b \leq m \leq n} y_{1}\left(p^{m}\right)_{k}^{(m-b, b)}
$$

identifying each $y_{1}\left(p^{m}\right)_{k}^{(m-b, b)}$ with a closed substack of $\mathcal{H}_{1}\left(p^{n}\right)_{k}$ via $\iota^{\left(p^{m}\right)}$. Each $y_{1}\left(p^{m}\right)_{k}^{(m-b, b)}$ is "set-theoretically identified with $Z_{b}$ " in the sense that $\left(Z_{b}\right)_{\mathrm{red}}=$ $y_{1}\left(p^{m}\right)_{k, \text { red }}^{(m-b, b)}$ as substacks of $\mathcal{H}_{1}\left(p^{n}\right)_{k, \text { red }}$ for all $b \leq m \leq n$.

To illustrate, visualize $\mathcal{H}_{1}\left(p^{n}\right)_{k \text {,red }}$ as follows:

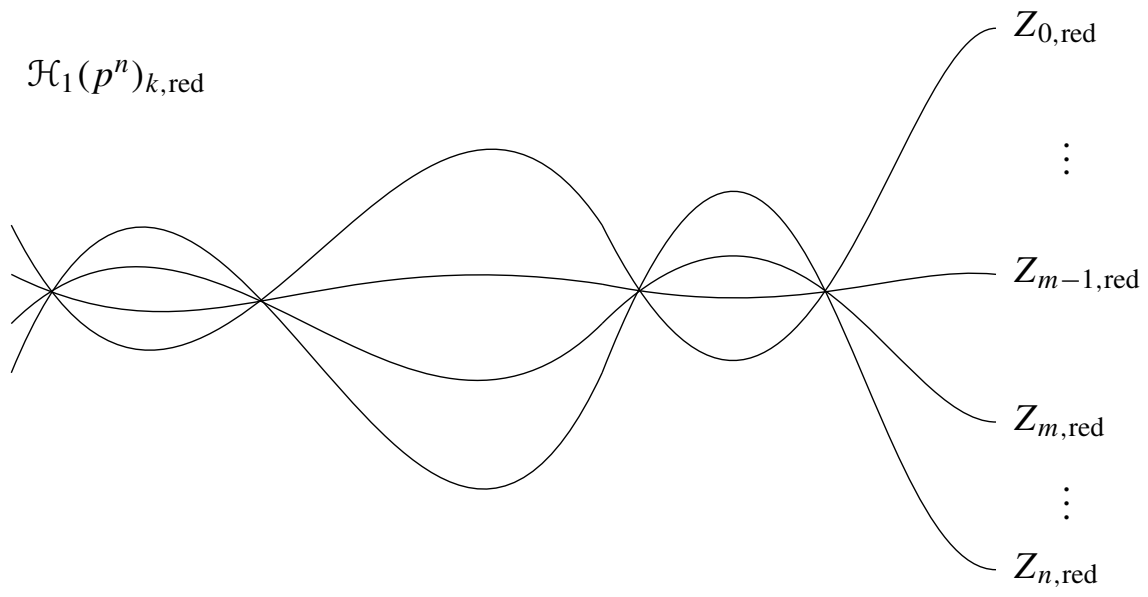

The closed immersion $\iota^{\left(p^{m}\right)}$ sends the following copy of $y_{1}\left(p^{m}\right)_{k}$ to the obvious closed substack of $\mathcal{H}_{1}\left(p^{n}\right)_{k}$, contributing nilpotent structure to the components $Z_{0}, \ldots, Z_{m}$ : 


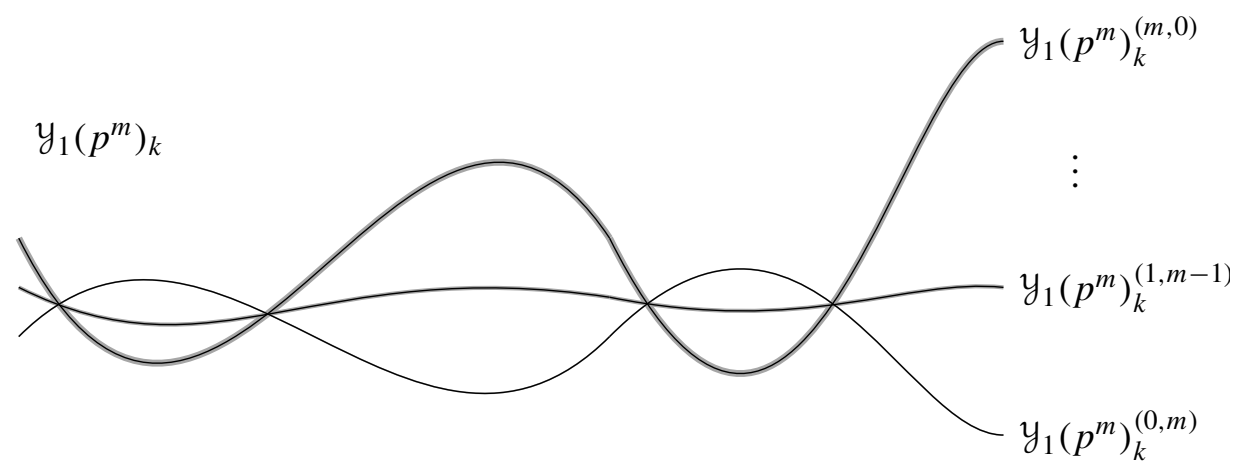

The result is that $\mathcal{H}_{1}\left(p^{n}\right)_{k \text {,red }} \cup y_{1}\left(p^{m}\right)_{k} \subseteq \mathcal{H}_{1}\left(p^{n}\right)_{k}$ looks something like this:

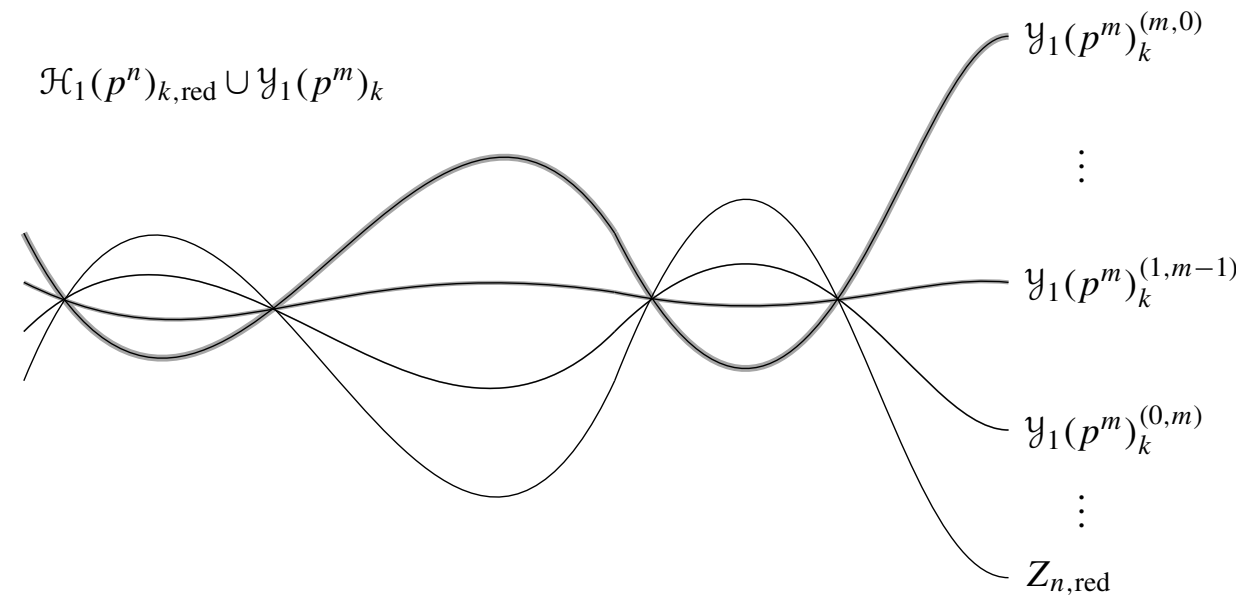

Each $y_{1}\left(p^{m}\right)_{k}$ (for $\left.0 \leq m \leq n\right)$ contributes additional nilpotent structure, giving us:

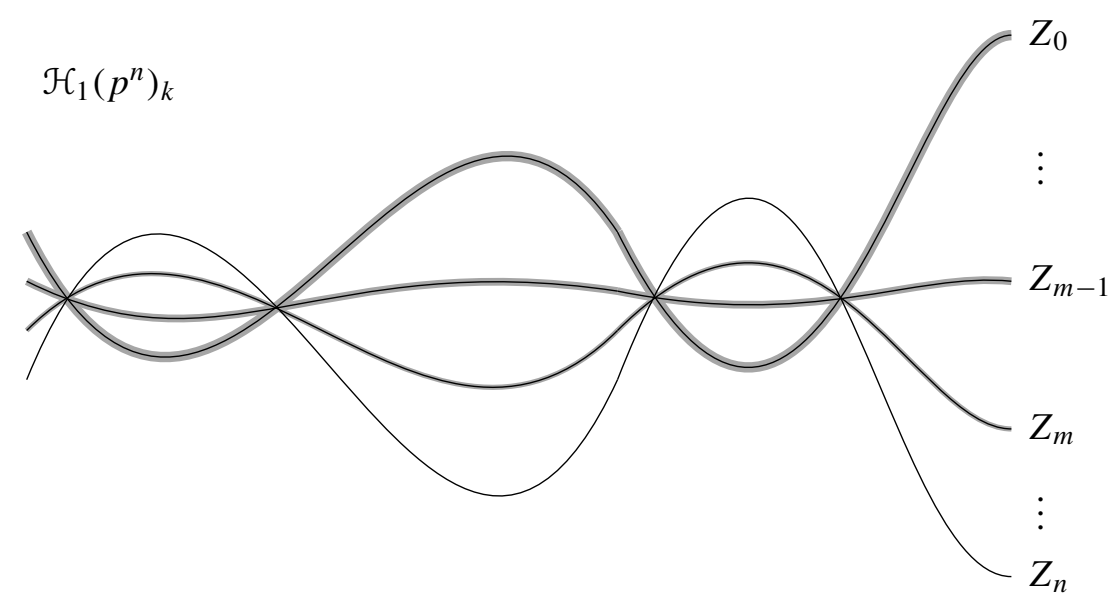


More generally, for arbitrary $N$ we get a closed immersion

$$
\iota^{(d)}: y_{1}(d) \hookrightarrow \mathcal{H}_{1}(N)
$$

for each $d$ dividing $N$, and the resulting map

$$
\bigsqcup_{d \mid N} y_{1}(d) \rightarrow \mathcal{H}_{1}(N)
$$

is an isomorphism over $S[1 / N]$. It follows immediately from [Katz and Mazur 1985, $\S 13.5]$ that if $(r, p)=1$, then for a perfect field $k$ of characteristic $p, y_{1}\left(p^{m} r\right)_{k}$ is the disjoint union, with crossings at the supersingular points, of $m+1$ components $y_{1}\left(p^{m} r\right)_{k}^{(m-b, b)}(0 \leq b \leq m)$, where

$$
y_{1}\left(p^{m} r\right)_{k}^{(m-b, b)}:=y_{1}(r)_{k} \times \overline{\mathcal{M}}_{1,1, k} y_{1}\left(p^{m}\right)_{k}^{(m-b, b)} .
$$

Now let $N=p^{n} N^{\prime}$, where $\left(N^{\prime}, p\right)=1$, and for any $r \mid N^{\prime}$ let $\mathcal{H}_{1}(N)_{k}^{r} \subset \mathcal{H}_{1}(N)_{k}$ denote the union of the components $y_{1}\left(p^{m} r\right)_{k}$ for $0 \leq m \leq n$. In summary:

Corollary 4.2. Let $k$ be a perfect field of characteristic $p$. For any $r$ dividing $N^{\prime}, \mathcal{H}_{1}(N)_{k}^{r}$ is the disjoint union, with crossings at the supersingular points, of components $Z_{b}^{r}$ for $0 \leq b \leq n$, where

$$
Z_{b}^{r}=\bigcup_{b \leq m \leq n} y_{1}\left(p^{m} r\right)_{k}^{(m-b, b)},
$$

identifying each $y_{1}\left(p^{m} r\right)_{k}^{(m-b, b)}$ with a closed substack of $\mathcal{H}_{1}(N)_{k}$ via $\iota^{\left(p^{m} r\right)}$. Each $y_{1}\left(p^{m} r\right)_{k}^{(m-b, b)}$ is "set-theoretically identified with $Z_{b}^{r}$ " in the sense that $\left(Z_{b}\right)_{\mathrm{red}}=$ $y_{1}\left(p^{m} r\right)_{k, \text { red }}^{(m-b, b)}$ as substacks of $\mathcal{H}_{1}(N)_{k \text {,red }} \mathcal{H}_{1}(N)_{k}$ is the disjoint union of the closed substacks

$$
\left\{\mathcal{H}_{1}(N)_{k}^{r}\right\}_{r \mid N^{\prime}}
$$

Closure of $y_{1}(N)$ in $\overline{\mathscr{K}}_{1,1}\left(\mathscr{B} \mu_{N}\right)$. We now want to describe the closure of $y_{1}(N)$ in $\overline{\mathscr{K}}_{1,1}\left(\mathscr{\Re} \mu_{N}\right)$, as a moduli stack classifying twisted curves with extra structure. This is accomplished in [Abramovich et al. 2003, §5.2] over $\mathbb{Z}[1 / N]$; let us briefly recall how this is done.

Definition 4.3. Let $G$ be a finite group, viewed as a finite étale group scheme over $\mathbb{Z}[1 /|G|]$. Fix an isomorphism $G^{*} \cong G$, after adjoining the necessary roots of unity to $\mathbb{Z}[1 /|G|]$. $\mathcal{B}_{g, n}^{\text {tei }}(G)$ is defined as the substack of $\overline{\mathscr{K}}_{1,1}(\mathscr{B} G)$ over $\mathbb{Z}[1 /|G|]$ whose objects are twisted Teichmüller $G$-structures on twisted curves. An object of $\overline{\mathscr{K}}_{g, n}(\mathscr{B} G)(T)$ is a pair $(\mathscr{C}, \phi)$, where $\mathscr{C} / T$ is a 1 -marked genus- 1 twisted stable curve with nonstacky marking, and $\phi$ is a group scheme homomorphism $G \rightarrow$ $\operatorname{Pic}_{\mathscr{C} / T}^{0}$. By definition, $(\mathscr{C}, \phi) \in \mathcal{B}_{g, n}^{\text {tei }}(G)(T)$ if and only if, whenever $P \rightarrow \mathscr{C}$ is a $G$-torsor corresponding to $\phi$ (fppf locally on $T$ ), the geometric fibers of $P / T$ are connected. 
$\mathcal{B}_{g, n}^{\text {tei }}(G)$ is naturally a closed substack of $\overline{\mathscr{K}}_{g, n}(\mathscr{B} G)$. Working over $\mathbb{Z}[1 / N]$, the choice of a primitive $N$-th root of unity $\zeta_{N}$ determines an isomorphism of group schemes $G:=\mathbb{Z} /(N) \cong \mu_{N}$ over $\mathbb{Z}\left[\zeta_{N}, 1 / N\right]$. Applying the resulting isomorphism $\overline{\mathscr{K}}_{1,1}(\mathscr{B} G) \cong \overline{\mathscr{K}}_{1,1}\left(\mathscr{B} \mu_{N}\right)$, we may view $y_{1}(N)$ as a substack of $\overline{\mathscr{K}}_{1,1}(\mathscr{B} G)$, and the closure of $y_{1}(N)$ in $\overline{\mathscr{K}}_{1,1}(\mathscr{B} G)$ over $\mathbb{Z}\left[\zeta_{N}, 1 / N\right]$ can be shown to be $\mathcal{B}_{1,1}^{\text {tei }}(G)$ (indeed, this follows from Theorem 4.6). Thus:

Corollary 4.4. The closure of $y_{1}(N)$ in $\overline{\mathscr{K}}_{1,1}\left(\mathscr{B} \mu_{N}\right)$ over $\mathbb{Z}[1 / N]$ is the stack whose objects over a scheme $T / \mathbb{Z}[1 / N]$ are pairs $(\mathscr{C}, \phi)$, where $\mathscr{C} / T$ is a 1 -marked genus-1 twisted stable curve with nonstacky marking, $\phi: \mathbb{Z} /(N) \rightarrow \operatorname{Pic}_{\mathscr{C} / S}^{0}$ is a group scheme homomorphism, and whenever $P \rightarrow \mathscr{C}$ is a $\mu_{N}$-torsor corresponding to $\phi$ (fppf locally on $T$ ) the geometric fibers of $P / T$ are connected.

However, in characteristics dividing $N$, simply requiring the $\mu_{N}$-torsors to have connected geometric fibers will not give us a moduli stack isomorphic to the closure of $y_{1}(N)$ in $\overline{\mathscr{K}}_{1,1}\left(\mathscr{B} \mu_{N}\right)$. For example, the group scheme $\mu_{p^{n}}$ is itself connected

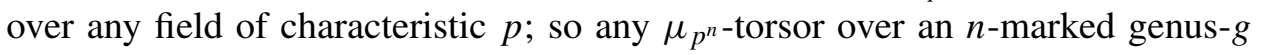
twisted stable curve over a field of characteristic $p$ will automatically be connected. So if the above result held over a base scheme $S \in \operatorname{Sch} / \mathbb{F}_{p}$, it would say the closure of $y_{1}\left(p^{n}\right)$ in $\overline{\mathscr{K}}_{1,1}\left(\mathscr{B} \mu_{p^{n}}\right)$ is all of $\overline{\mathscr{K}}_{1,1}^{\prime}\left(\mathscr{B} \mu_{p^{n}}\right)$ (the substack of $\overline{\mathscr{K}}_{1,1}\left(\mathscr{B} \mu_{p^{n}}\right)$ where the marking is representable). We will see that this is not true; the closure of $y_{1}\left(p^{n}\right)$ turns out to be finite and flat of constant rank $p^{2 n}-p^{2 n-2}$ over $\bar{M}_{1,1}$, while $\overline{\mathscr{K}}_{1,1}^{\prime}\left(\mathscr{B} \mu_{p^{n}}\right)$ turns out to be finite and flat of constant rank $p^{2 n}$ over $\bar{M}_{1,1}$.

A $\mu_{N}$-torsor over $\mathscr{C} / S$ determines a group scheme homomorphism $\mathbb{Z} /(N) \rightarrow$ $\mathrm{Pic}_{\mathscr{C} / S}^{0}$ (see Lemma 3.19), and over $\mathbb{Z}[1 / N]$ a $\mu_{N}$-torsor with connected geometric fibers corresponds to a group scheme homomorphism which is injective. We are therefore led to consider Drinfeld structures on our twisted curves:

Definition 4.5. Let $\mathscr{b} / S$ be a 1 -marked genus-1 twisted stable curve with no stacky structure at its marking. A $\left[\Gamma_{1}(N)\right]$-structure on $\mathscr{C}$ is a group scheme homomorphism $\phi: \mathbb{Z} /(N) \rightarrow \mathrm{Pic}_{\mathscr{C} / S}^{0}$ such that:

- the relative effective Cartier divisor

$$
D:=\sum_{a \in \mathbb{Z} /(N)}[\phi(a)]
$$

in $\operatorname{Pic}_{\mathscr{C} / S}^{0}$ is an $S$-subgroup scheme and

- for every geometric point $\bar{p} \rightarrow S, D_{\bar{p}}$ meets every irreducible component of $\left(\mathrm{Pic}_{\mathscr{C} / S}^{0}\right)_{\bar{p}}=\mathrm{Pic}_{\mathscr{C}_{\bar{p}} / k(\bar{p})}^{0}$.

Over any scheme $S$, we define $X_{1}^{\mathrm{tw}}(N)$ to be the substack of $\overline{\mathscr{K}}_{1,1}\left(\mathscr{B} \mu_{N}\right)$ whose objects over an $S$-scheme $T$ are pairs $(\mathscr{C}, \phi) \in \overline{\mathscr{K}}_{1,1}\left(\mathscr{B} \mu_{N}\right)(T)$ such that $\phi$ is a $\left[\Gamma_{1}(N)\right]$-structure on $\mathscr{C}$. 
Note that if $\mathscr{C} / S$ is a 1 -marked genus-1 stable curve (nontwisted), so that every geometric fiber of $\mathscr{C} / S$ is either a smooth elliptic curve or a Néron 1-gon, then this agrees with the definition of a $\left[\Gamma_{1}(N)\right]$-structure on $\mathscr{C} / S$ as given in [Conrad 2007, 2.4.1] and in Definition 2.3 above, identifying a $\left[\Gamma_{1}(N)\right]$-structure $P \in \mathscr{C}^{\mathrm{sm}}(S)$ with its corresponding group scheme homomorphism $\mathbb{Z} /(N) \rightarrow \mathscr{C}^{\mathrm{sm}}, 1 \mapsto P$, since $\mathrm{Pic}_{\mathscr{C} / S}^{0} \cong \mathscr{C}^{\text {sm }}$ in this case.

Theorem 4.6 (Restatement of Theorem 1.1). Let $S$ be a scheme and $X_{1}^{\mathrm{tw}}(N)$ the stack over $S$ classifying $\left[\Gamma_{1}(N)\right]$-structures on 1-marked genus-1 twisted stable curves with nonstacky marking. Then $\mathcal{X}_{1}^{\mathrm{tw}}(N)$ is a closed substack of $\overline{\mathscr{K}}_{1,1}\left(\Re_{\mu_{N}}\right)$, which contains $y_{1}(N)$ as an open dense substack.

In particular $X_{1}^{\mathrm{tw}}(N)$ is flat over $S$ with local complete intersection fibers, and is proper and quasifinite over $\bar{M}_{1,1}$.

Remark 4.7. Although this gives a new modular compactification of $y_{1}(N)$, it should be noted that the proof of the theorem relies in several places on the proof in [Conrad 2007] that the moduli stack classifying $\left[\Gamma_{1}(N)\right]$-structures on generalized elliptic curves is a proper algebraic stack over $\bar{M}_{1,1}$.

Proof of Theorem 4.6. The main point is to verify the valuative criterion of properness for $X_{1}^{\mathrm{tw}}(N)$, which implies $X_{1}^{\mathrm{tw}}(N)$ is a closed substack of $\overline{\mathscr{K}}_{1,1}\left(\Re_{\mu_{N}}\right)$. It follows from Lemma 3.21 that $y_{1}(N)$ is dense.

Let $R$ be a discrete valuation ring with $T:=\operatorname{Spec}(R) \in \operatorname{Sch} / S$; write $\eta=$ $\operatorname{Spec}(K)$ for the generic point of $T$ and $s=\operatorname{Spec}(k)$ for the closed point. Let $\left(\mathscr{C}_{\eta}, \phi_{\eta}\right) \in X_{1}^{\mathrm{tw}}(N)(\eta)$, so $\mathscr{C}_{\eta} / K$ is a 1 -marked genus- 1 twisted stable curve with nonstacky marking and $\phi_{\eta}: \mathbb{Z} /(N) \rightarrow \operatorname{Pic}_{\mathscr{C}_{\eta} / K}^{0}$ is a $\left[\Gamma_{1}(N)\right]$-structure on $\mathscr{C}_{\eta}$. Since $\overline{\mathscr{K}}_{1,1}\left(\Re \mu_{N}\right)$ is proper over $S$, there is a discrete valuation ring $R_{1}$ containing $R$ as a local subring, with corresponding morphism of spectra $T_{1} \rightarrow T$ over $S$, such that the pair $\left(\mathscr{C}_{\eta} \times_{T} T_{1},\left(\phi_{\eta}\right)_{T_{1}}\right)$ extends to a pair $\left(\mathscr{C}_{T_{1}}, \phi_{T_{1}}\right) \in \overline{\mathscr{K}}_{1,1}\left(\mathscr{B}_{\mu_{N}}\right)(T)$; whenever such an extension exists, it is unique. Therefore it suffices to show that for some such $R_{1}$ and $T_{1}$, there exists a $\left[\Gamma_{1}(N)\right]$-structure $\phi_{T_{1}}$ on a 1-marked genus-1 twisted stable curve $\mathscr{C}_{T_{1}}$ extending the $\left[\Gamma_{1}(N)\right]$-structure $\left(\phi_{\eta}\right)_{T_{1}}$ on $\mathscr{C}_{\eta} \times_{T} T_{1}$. This is accomplished in Lemmas 4.9-4.12.

Terminology 4.8. In the following lemmas and their proofs, "base change on $R$ " will refer to replacing $R$ with a discrete valuation ring $R_{1}$ as above.

By [Deligne and Rapoport 1973, IV.1.6], after a base change on $R$ we may assume that the minimal proper regular model of the coarse space $C_{\eta}$ of $\mathscr{C}_{\eta}$ is a generalized elliptic curve. We maintain this assumption for the rest of the proof.

Lemma 4.9. Suppose $\mathscr{C}_{\eta} / K$ is a smooth elliptic curve whose minimal proper regular model over $R$ is smooth, and $\phi_{\eta}$ is a $\left[\Gamma_{1}(N)\right]$-structure on $\mathscr{C}_{\eta}$. Then after 
base change on $R,\left(\mathscr{C}_{\eta}, \phi_{\eta}\right)$ extends to a $\left[\Gamma_{1}(N)\right]$-structure $\phi$ on a smooth elliptic curve $\mathscr{C} / R$.

Proof. This follows immediately from the fact that the stack $X_{1}(N)$ classifying $\left[\Gamma_{1}(N)\right]$-structures on generalized elliptic curves is proper [Conrad 2007, 1.2.1].

Lemma 4.10. Suppose $\left(\mathfrak{C}_{\eta}, \phi_{\eta}\right) \in X_{1}^{\mathrm{tw}}(N)(\eta)$, such that the coarse space $C_{\eta}$ of $\mathscr{C}_{\eta}$ is singular. Then after base change on $R,\left(\mathscr{C}_{\eta}, \phi_{\eta}\right)$ extends to $(\mathscr{C}, \phi) \in X_{1}^{\mathrm{tw}}(N)(R)$.

Proof. After a base change on $R$, we may assume that $\mathscr{C}_{\eta} / K$ is the standard $\mu_{d}$-stacky Néron 1-gon as in Example 3.4, for some $d \geq 1$; since $\mathscr{C}_{\eta}$ admits a $\left[\Gamma_{1}(N)\right]$-structure and $\mathrm{Pic}_{\mathscr{C}_{\eta} / K}^{0} \cong \mathbb{G}_{m, K} \times \mathbb{Z} /(d)$, we have $d \mid N$.

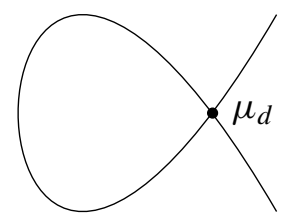

Standard $\mu_{d}$-stacky Néron 1-gon

Let $\mathscr{C} / R$ be the standard $\mu_{d}$-stacky Néron 1-gon over $R$, so $\mathscr{C}$ is a genus- 1 twisted curve over $R$ extending $\mathscr{C}_{\eta}$ (and of course the marking of $\mathscr{C}_{\eta}$ extends to $\mathscr{C}$ ). After further base change on $R$ we may assume $\mu_{N}(K)=\mu_{N}(R)$. Let $\phi_{\eta}(1)=(\zeta, a) \in$ $\mathbb{G}_{m}(K) \times \mathbb{Z} /(d)$; since $\phi_{\eta}$ is a $\left[\Gamma_{1}(N)\right]$-structure on $\mathscr{b}_{\eta}$, we have $\zeta \in \mu_{N}(K)$ and $a \in$ $(\mathbb{Z} /(d))^{\times}$. Since $\mu_{N}(K)=\mu_{N}(R)$, this extends to a section $(\zeta, a) \in \mathbb{G}_{m}(R) \times \mathbb{Z} /(d)$, determining a group scheme homomorphism $\phi: \mathbb{Z} /(N) \rightarrow \mathbb{G}_{m, R} \times \mathbb{Z} /(d)$ with $\phi(1)=(\zeta, a)$. Since $a \in(\mathbb{Z} /(d))^{\times}, \phi$ is a $\left[\Gamma_{1}(N)\right]$-structure on $\mathscr{C}$.

Lemma 4.11. Suppose $R$ has pure characteristic $p \geq 0$, and $\mathscr{C}_{\eta}=E_{\eta}$ is an elliptic curve over $K$ whose minimal proper regular model over $R$ is not smooth; that is, $E_{\eta} / K$ is an ordinary elliptic curve with bad reduction. If $\phi_{\eta}: \mathbb{Z} /(N) \rightarrow \operatorname{Pic}_{\mathscr{C}_{\eta} / K}^{0}$ is a $\left[\Gamma_{1}(N)\right]$-structure on $\mathscr{C}_{\eta}$, then after base change on $R$, there exists a pair $(\mathscr{C}, \phi) \in X_{1}^{\mathrm{tw}}(N)(R)$ extending $\left(\mathscr{C}_{\eta}, \phi_{\eta}\right)$.

Proof. Since for any coprime $N$ and $N^{\prime}$ we obviously have

$$
\overline{\mathscr{K}}_{1,1}\left(\mathscr{B} \mu_{N N^{\prime}}\right) \cong \overline{\mathscr{K}}_{1,1}\left(\mathscr{B} \mu_{N}\right) \times_{\bar{M}_{1,1}} \overline{\mathscr{K}}_{1,1}\left(\mathscr{B} \mu_{N^{\prime}}\right)
$$

and

$$
X_{1}^{\mathrm{tw}}\left(N N^{\prime}\right) \cong X_{1}^{\mathrm{tw}}(N) \times_{\bar{M}_{1,1}} X_{1}^{\mathrm{tw}}\left(N^{\prime}\right),
$$

it suffices to consider the cases where (a) $N$ is prime to $p$ (including the case $p=0$ ) and (b) $N=p^{n}$.

(a) First suppose $(N, p)=1$ or $p=0$. After base extension on $R$ we may assume that the finite abelian group $E_{\eta}(K)[N]$ is isomorphic to $(\mathbb{Z} /(N))^{2}$. After further base extension on $R$, the map $\phi_{\eta}$ gives us a $\mu_{N}$-torsor $P_{\eta} \rightarrow E_{\eta}$, corresponding to the 
point $Q_{\eta}=\phi_{\eta}(1) \in \operatorname{Pic}_{E_{\eta} / K}^{0}(K) \cong E_{\eta}(K)$ of "exact order $N$ " in the sense of [Katz and Mazur 1985, §1.4] (and since $(N, p)=1$, this just says $Q_{\eta}$ has exact order $N$ as an element of the group $\left.E_{\eta}(K)\right)$. Let $\mathcal{E} / R$ be the minimal proper regular model; after further base extension on $R$ and replacing $\mathcal{E}$ with the new minimal proper regular model of $E_{\eta}$, we may assume $\mathcal{E}$ has the structure of a generalized elliptic curve extending that of $E_{\eta}$ (see [Deligne and Rapoport 1973, IV.1.6], and also [Conrad 2007, proof of 3.2.6]). Since $E_{\eta}(K)=\mathcal{E}^{\mathrm{sm}}(R)$, the finite flat $R$-group scheme $\mathcal{E}^{\mathrm{sm}}[N]$ has rank $N^{2}$, hence is étale over $\operatorname{Spec}(R)$ since $(N, p)=1$. Therefore the special fiber $\mathcal{E}_{s}$ is geometrically a Néron $m N$-gon for some $m$. Replacing $\mathcal{E}$ with its contraction away from the subgroup scheme $\mathcal{E}^{\mathrm{sm}}[N] \subset \mathcal{E}^{\mathrm{sm}}$ (see [Deligne and Rapoport 1973, IV.1]), we get a generalized elliptic curve $\mathcal{E} / R$ extending $E_{\eta}$, whose special fiber is geometrically a Néron $N$-gon, with $E_{\eta}(K)[N] \cong \mathcal{E}^{\mathrm{sm}}(R)[N]$.
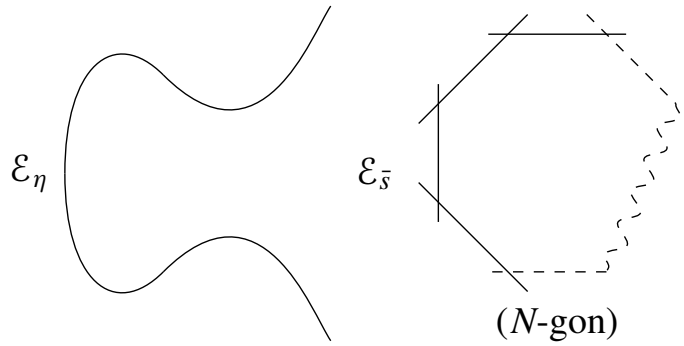

Note that $Q_{\eta}$ extends uniquely to a section $Q \in \mathcal{E}^{\mathrm{sm}}(R)[N]$ of exact order $N$, which Conrad calls a "possibly nonample $\left[\Gamma_{1}(N)\right]$-structure on $\mathcal{E}$ ", meaning $Q$ satisfies all the conditions of a $\left[\Gamma_{1}(N)\right]$-structure except that the relative effective Cartier divisor $\sum_{a \in \mathbb{Z} /(N)}[a \cdot Q]$ might not meet every irreducible component of a geometric closed fiber $\mathcal{E}_{\bar{s}}$.

Recall that by Corollary 3.20,

$$
P_{\eta}=\underline{\operatorname{Spec}}_{E_{\eta}}\left(\bigoplus_{a=0}^{N-1} \mathscr{L}\left(\left(a \cdot Q_{\eta}\right)-\left(0_{E_{\eta}}\right)\right)\right)
$$

with the $\mu_{N}$-action on $\bigoplus \mathscr{L}\left(\left(a \cdot Q_{\eta}\right)-\left(0_{E_{\eta}}\right)\right)$ induced by the $\mathbb{Z} /(N)$-grading. Since $N$ is invertible on $\operatorname{Spec}(R)$, the assumptions of Lemma 3.22 are satisfied, so we may identify $P_{\eta}$ with $E_{\eta} /\left\langle Q_{\eta}\right\rangle$, with the quotient map $P_{\eta} \rightarrow E_{\eta}$ identified with the quotient map $E_{\eta} /\left\langle Q_{\eta}\right\rangle \rightarrow E_{\eta} / E_{\eta}[N] \cong E_{\eta}$. Then the $\mu_{N}$-action on $P_{\eta}=E_{\eta} /\left\langle Q_{\eta}\right\rangle$ is determined by the group scheme isomorphism $E_{\eta}[N] /\left\langle Q_{\eta}\right\rangle \cong \mu_{N}$ induced by the $e_{N}$-pairing and the choice of $Q_{\eta}$ :

$$
E_{\eta}[N] /\left\langle Q_{\eta}\right\rangle=\left\{Q_{\eta}\right\} \times E_{\eta}[N] /\left\langle Q_{\eta}\right\rangle \hookrightarrow\left\langle Q_{\eta}\right\rangle \times E_{\eta}[N] /\left\langle Q_{\eta}\right\rangle \stackrel{e_{N}}{\rightarrow} \mu_{N} .
$$

By [Conrad 2007, Theorem 4.1.1], the $e_{N}$-pairing on $E_{\eta} / K$ extends (possibly after further base change on $R$ ) to a nondegenerate bilinear pairing of finite flat 
commutative group schemes over $R$

$$
e_{N}: \mathcal{E}^{\mathrm{sm}}[N] \times \mathcal{E}^{\mathrm{sm}}[N] \rightarrow \mu_{N}
$$

Therefore the isomorphism of group schemes $E_{\eta}[N] /\left\langle Q_{\eta}\right\rangle \cong \mu_{N}$ described above may be extended via the same formula to an isomorphism of group schemes $\varepsilon^{\mathrm{sm}}[N] /\langle Q\rangle \cong \mu_{N}$.

This isomorphism of group schemes makes $P^{\prime}:=[\mathcal{E} /\langle Q\rangle]$ a $\mu_{N}$-torsor over the twisted curve $\mathscr{C}^{\prime}:=\left[\mathcal{E} / \mathcal{E}^{\mathrm{sm}}[N]\right]$, extending our original $\mu_{N}$-torsor over $E_{\eta}\left(\mathscr{C}^{\prime}\right.$ is indeed a twisted curve: by [Abramovich et al. 2011, Proposition 2.3] we may detect this on the geometric fibers, where it is clear, since the geometric closed fiber of $\mathscr{C}^{\prime}$ is a standard $\mu_{N}$-stacky Néron 1-gon). But $P^{\prime}$ is not necessarily representable. Indeed, let $d$ be the minimal positive integer such that $d \cdot Q_{\bar{s}}$ lies in the identity component of the geometric closed fiber $\mathcal{E}_{\bar{s}}$. Then the coarse space $\bar{P}_{\bar{s}}^{\prime}$ of $P_{\bar{s}}^{\prime}$ is a Néron $N / d$-gon, and for any geometric point $\bar{q} \rightarrow \bar{P}_{\bar{s}}^{\prime}$ mapping to a node of $\bar{P}_{\bar{s}}^{\prime}$, we have $P_{\bar{s}}^{\prime} \times \bar{P}_{\bar{s}}^{\prime}, \bar{q} \cong\left(\mathscr{B} \mu_{N / d}\right)_{k(\bar{q})}$.
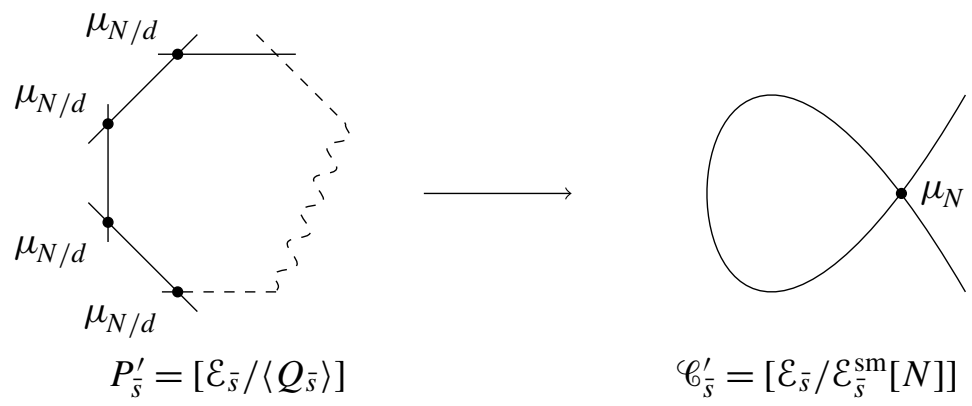

(Stacky $N / d$-gon)

Let $P \rightarrow \mathscr{C}$ be the $\mu_{N}$-torsor corresponding to the relative coarse moduli space [Abramovich et al. 2011, Theorem 3.1] of the map $\mathscr{C}^{\prime} \rightarrow \mathscr{B} \mu_{N}$ coming from the $\mu_{N}$-torsor $P^{\prime} \rightarrow \mathscr{C}^{\prime}$. The generic fibers are the same as those of $P^{\prime} \rightarrow \mathscr{C}^{\prime}, P_{\bar{s}}$ is a nonstacky Néron $N / d$-gon, and $\mathscr{C}_{\bar{s}}$ is a standard $\mu_{d}$-stacky Néron 1-gon, say with coarse space $\pi: \mathscr{C}_{\bar{s}} \rightarrow C_{\bar{s}}$.

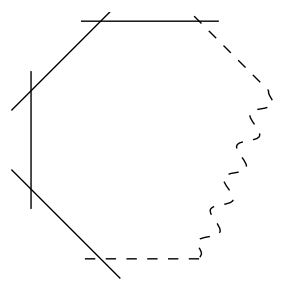

$P_{\bar{s}}$

$(N / d$-gon $)$

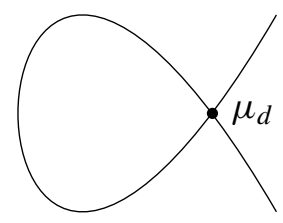

$\varphi_{\bar{s}}$ 
Let $\bar{q} \rightarrow C_{\bar{s}}$ be a geometric point mapping to the node of $C_{\bar{s}}$. Then $\mathscr{C}_{\bar{s}} \times_{C_{\bar{s}}} \bar{q}$ $\cong\left(\mathscr{B} \mu_{d}\right)_{k(\bar{q})}$ and $P_{\bar{s}} \times_{C_{\bar{s}}} \bar{q}=\mu_{N / d} \times \bar{q}$ (which as a $k(\bar{q})$-scheme is just $N / d$ disjoint copies of $\bar{q}$ since $(N, p)=1)$, so the resulting $\mu_{N}$-torsor over $\left(\mathscr{B}_{d} \mu_{k}\right)_{k}(\bar{q})$ corresponds to a generator of $H^{1}\left(\mathscr{B} \mu_{d}, \mu_{N}\right) \cong \mathbb{Z} /(d)$. Therefore, with respect to the decomposition

$$
\operatorname{Pic}_{\mathscr{C}_{\bar{s}} / k(\bar{s})}^{0} \cong \operatorname{Pic}_{C_{\bar{s}} / k(\bar{s})}^{0} \times H^{0}\left(C_{\bar{s}}, \boldsymbol{R}^{1} \pi_{*} \mathbb{G}_{m}\right) \cong \mathbb{G}_{m} \times \mathbb{Z} /(d),
$$

the class of the $\mu_{N}$-torsor $P_{\bar{s}} \rightarrow \mathscr{C}_{\bar{s}}$ projects in the second factor to a generator of $\mathbb{Z} /(d)$, so the map $\phi: \mathbb{Z} /(N) \rightarrow \operatorname{Pic}_{\mathscr{C} / R}^{0}$ induced by $P$ is a $\left[\Gamma_{1}(N)\right]$-structure on the twisted curve $\mathscr{C} / R$ extending the $\left[\Gamma_{1}(N)\right]$-structure $\phi_{\eta}$ on $\mathscr{C}_{\eta}$.

(b) Now suppose $N=p^{n}$. After base change on $R$, the $\left[\Gamma_{1}(N)\right]$-structure $\phi_{\eta}$ gives us a $\mu_{N}$-torsor $P_{\eta} \rightarrow E_{\eta}$ corresponding to the point $Q_{\eta}=\phi_{\eta}(1) \in \operatorname{Pic}_{E_{\eta} / K}^{0}(K) \cong E_{\eta}(K)$ of "exact order $N$ " (in the sense of [Katz and Mazur 1985, §1.4], but not necessarily as an element of the group $\left.E_{\eta}(K)\right)$. Since $E_{\eta} / K$ is ordinary, after base change on $R$ we have an isomorphism of group schemes over $K$

$$
E_{\eta}[N] \cong \mu_{N} \times \mathbb{Z} /(N),
$$

so $E_{\eta}(K)[N] \cong \mu_{N}(K) \times \mathbb{Z} /(N)=\{1\} \times \mathbb{Z} /(N)=\mathbb{Z} /(N)$. The $\mu_{N}$-torsor $P_{\eta} \rightarrow E_{\eta}$ is

$$
P_{\eta}=\underline{\operatorname{Spec}}_{E_{\eta}}\left(\bigoplus_{a=0}^{N-1} \mathscr{L}\left(\left(a \cdot Q_{\eta}\right)-\left(0_{E_{\eta}}\right)\right)\right)
$$

with the $\mu_{N}$-action on $\bigoplus \mathscr{L}\left(\left(Q_{\eta}\right)-\left(0_{E_{\eta}}\right)\right)$ induced by the $\mathbb{Z} /(N)$-grading.

Choose $m \geq 0$ minimal such that the image of $p^{m} \cdot Q_{\eta}$ in $\mathbb{Z} /(N)$ is zero. So $p^{m} \cdot Q_{\eta}=0_{E_{\eta}}$ is viewed as a point of "exact order $p^{n-m}$ " on $E_{\eta}$. Since $\mathscr{L}\left(\left(p^{m} \cdot Q_{\eta}\right)\right.$

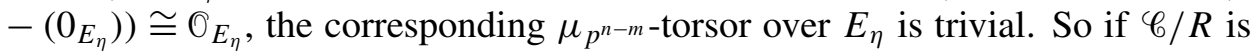

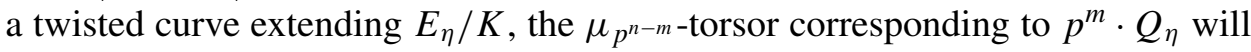

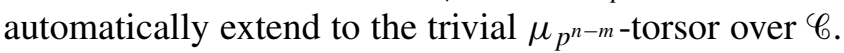

Now view $Q_{\eta}$ as a point of "exact order $p^{m}$ " on $E_{\eta} / K$. The relative effective Cartier divisor

$$
D_{\eta}:=\sum_{a \in \mathbb{Z} /\left(p^{m}\right)}\left[a \cdot Q_{\eta}\right]
$$

in $E_{\eta}$ is a subgroup scheme which is étale over $K$. So by Lemma 3.22, $E_{\eta} / D_{\eta}$ is

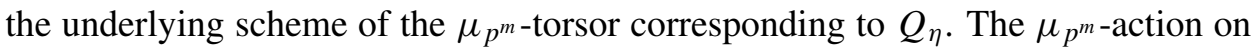
$E_{\eta} / D_{\eta}$ is given by the group scheme isomorphism

$$
E_{\eta}\left[p^{m}\right] / D_{\eta} \cong \mu_{p^{m}}
$$

induced by the $e_{p^{m}}$-pairing and the choice of $Q_{\eta}$ :

$$
E_{\eta}\left[p^{m}\right] / D_{\eta}=\left\{Q_{\eta}\right\} \times E_{\eta}\left[p^{m}\right] / D_{\eta} \hookrightarrow D_{\eta} \times E_{\eta}\left[p^{m}\right] / D_{\eta} \stackrel{e_{p^{m}}}{\rightarrow} \mu_{p^{m}} .
$$


After a base change on $R$ if necessary, let $\mathcal{E} / R$ be a generalized elliptic curve extending $E_{\eta}$, with a $\left[\Gamma_{1}\left(p^{m}\right)\right]$-structure $Q$ on $\mathcal{E}$ extending $Q_{\eta}$. The special fiber $\mathcal{E}_{s} / k$ is geometrically a Néron $p^{m}$-gon, and $\mathcal{E}^{\mathrm{sm}}\left[p^{m}\right] \cong \mu_{p^{m}} \times \mathbb{Z} /\left(p^{m}\right)$.
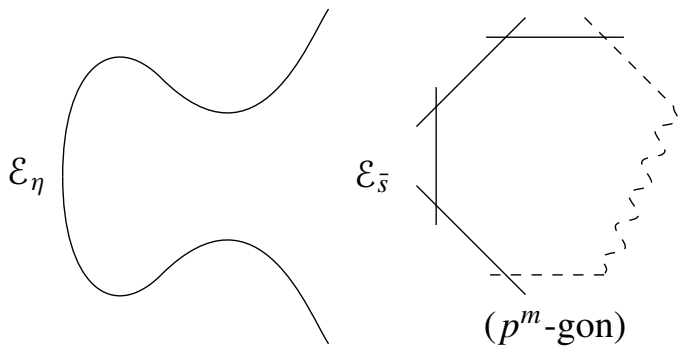

By [Conrad 2007, Theorem 4.1.1], the $e_{p^{m}}$-pairing on $E_{\eta} / K$ extends (possibly after further base change on $R$ ) to a nondegenerate bilinear pairing of finite flat commutative group schemes over $R$

$$
e_{p^{m}}: \mathcal{E}^{\mathrm{sm}}\left[p^{m}\right] \times \mathcal{E}^{\mathrm{sm}}\left[p^{m}\right] \rightarrow \mu_{p^{m}} .
$$

Therefore the isomorphism of group schemes $E_{\eta}\left[p^{m}\right] / D_{\eta} \cong \mu_{p^{m}}$ described above may be extended via the same formula to an isomorphism of group schemes $\mathcal{E}^{\mathrm{sm}}\left[p^{m}\right] / D \cong \mu_{p^{m}}$, where $D$ is the relative effective Cartier divisor

$$
D=\sum_{a \in \mathbb{Z} /\left(p^{m}\right)}[a \cdot Q]
$$

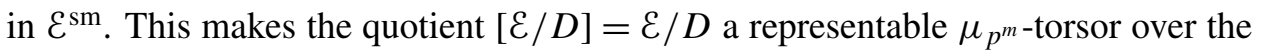
twisted curve $\mathscr{C}:=\left[\mathcal{E} / \mathcal{E}^{\mathrm{sm}}\left[p^{m}\right]\right]$, which extends the given $\mu_{p^{m}}$-torsor over $E_{\eta}$.

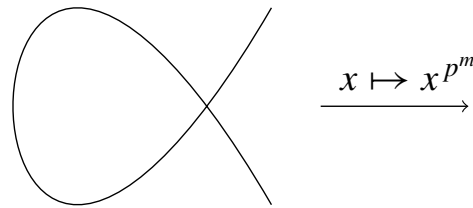

$\left[\mathcal{E}_{\bar{s}} / D_{\bar{s}}\right]$

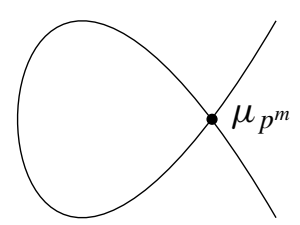

$$
\mathscr{C}_{\bar{s}}=\left[\mathcal{E}_{\bar{s}} / \mathcal{E}_{\bar{s}}^{\mathrm{sm}}\left[p^{m}\right]\right]
$$

The geometric special fiber $\mathscr{C}_{\bar{s}}$ is a standard $\mu_{p^{m}}$-stacky Néron 1-gon, say with coarse space $\pi: \mathscr{C}_{\bar{s}} \rightarrow C_{\bar{s}} ;\left[\mathcal{E}_{\bar{s}} / D_{\bar{s}}\right]=\mathcal{E}_{\bar{s}} / D_{\bar{s}}$ is a (nonstacky) Néron 1-gon, and the quotient map $P_{\bar{s}}:=\mathcal{E}_{\bar{s}} / D_{\bar{s}} \rightarrow \mathscr{C}_{\bar{s}}$ extends the map $P_{\bar{s}}^{\mathrm{sm}}=\mathbb{G}_{m} \rightarrow \mathscr{C}_{\bar{s}}^{\mathrm{sm}}=\mathbb{G}_{m}, x \mapsto x^{p^{m}}$. In particular, if $\bar{q} \rightarrow C_{\bar{s}}$ is a geometric point mapping to the node of $C_{\bar{s}}$, then

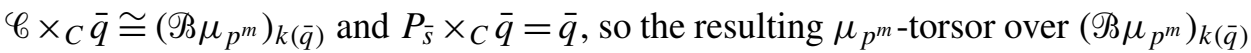
corresponds to a generator of $H^{1}\left(\mathscr{B} \mu_{p^{m}}, \mu_{p^{m}}\right) \cong \mathbb{Z} /\left(p^{m}\right)$. Therefore, with respect to the decomposition

$$
\operatorname{Pic}_{\mathscr{C}_{\bar{s}} / k(\bar{s})}^{0} \cong \operatorname{Pic}_{C_{\bar{s}} / k(\bar{s})}^{0} \times H^{0}\left(C_{\bar{s}}, \boldsymbol{R}^{1} \pi_{*} \mathbb{G}_{m}\right) \cong \mathbb{G}_{m} \times \mathbb{Z} /\left(p^{m}\right),
$$


the class of the $\mu_{p^{m}}$-torsor $P_{\bar{s}} \rightarrow \mathscr{C}_{\bar{s}}$ projects in the second factor to a generator of $\mathbb{Z} /\left(p^{m}\right)$, so the group scheme homomorphism $\phi: \mathbb{Z} /\left(p^{m}\right) \rightarrow \operatorname{Pic}_{\mathscr{C} / R}^{0}$ corresponding to $P:=[\mathcal{E} / D]=\mathcal{E} / D$ is a $\left[\Gamma_{1}\left(p^{m}\right)\right]$-structure on the twisted curve $\mathscr{C} / R$.

Finally, write

$$
P=\underline{\operatorname{Spec}}_{\bullet}\left(\bigoplus_{a=0}^{p^{m}-1} \mathscr{L}_{a}\right),
$$

with the grading determined by the $\mu_{p^{m}}$-action on $P$. Since $P$ extends the $\mu_{p^{m}}$-torsor on $E_{\eta}$ determined by $Q_{\eta}$, we have $\left.\mathscr{L}_{a}\right|_{\eta} \cong \mathscr{L}\left(\left(Q_{\eta}\right)-\left(0_{E_{\eta}}\right)\right)$. Then

$$
P \times \mu_{p^{n-m}}=\underline{\operatorname{Spec}} \mathscr{C}\left(\bigoplus_{a=0}^{p^{n}-1} \mathscr{L}_{\left(a \bmod p^{m}\right)}\right)
$$

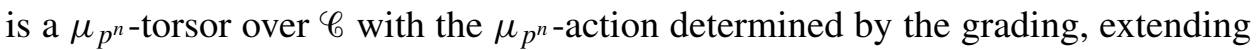
the original $\mu_{p^{n}}$-torsor over $E_{\eta}$ and representable since $P$ is representable. Since the group scheme homomorphism $\phi: \mathbb{Z} /\left(p^{m}\right) \rightarrow \operatorname{Pic}_{\mathscr{C} / R}^{0}$ corresponding to $P$ is a $\left[\Gamma_{1}\left(p^{m}\right)\right]$-structure on $\mathscr{C}$, and the group scheme homomorphism $\phi^{\prime}: \mathbb{Z} /\left(p^{n}\right) \rightarrow$ $\mathrm{Pic}_{\mathscr{C} / R}^{0}$ corresponding to $P \times \mu_{p^{n-m}}$ is $\phi \circ \pi$ for the canonical projection $\pi: \mathbb{Z} /\left(p^{n}\right) \rightarrow$ $\mathbb{Z} /\left(p^{m}\right)$, it follows immediately that $\phi^{\prime}$ is a $\left[\Gamma_{1}\left(p^{n}\right)\right]$-structure on $\mathscr{C}$.

Lemma 4.12. Suppose $R$ has mixed characteristic $(0, p)$, and $\mathscr{C}_{\eta}=E_{\eta}$ is an elliptic curve over $K$ whose minimal proper regular model over $R$ is not smooth; that is, $E_{\eta} / K$ is an ordinary elliptic curve with bad reduction. If $\phi_{\eta}: \mathbb{Z} /(N) \rightarrow \operatorname{Pic}_{\mathscr{C}_{\eta} / K}^{0}$ is a $\left[\Gamma_{1}(N)\right]$-structure on $\mathscr{C}_{\eta}$, then after base change on $R$, there exists a pair $(\mathscr{C}, \phi) \in X_{1}^{\mathrm{tw}}(N)(R)$ extending $\left(\mathscr{C}_{\eta}, \phi_{\eta}\right)$.

Proof. As before, we can restrict to the two separate cases, one where $(N, p)=1$ and another where $N=p^{n}$.

(a) If $(p, N)=1$, the same argument as in Part (a) of the proof of Lemma 4.11 carries through.

(b) Suppose $N=p^{n}$. As in the proof of Lemma 4.11, after base extension on $R$ we can extend $E_{\eta}$ to a generalized elliptic curve $\mathcal{E} / R$ whose special fiber is geometrically a Néron $N$-gon, such that $\mathcal{E}^{\mathrm{sm}}(R)[N] \cong E_{\eta}(K)[N] \cong(\mathbb{Z} /(N))^{2}$ (the latter isomorphism being a noncanonical isomorphism of abelian groups). After further base change on $R$, the $\left[\Gamma_{1}\left(p^{n}\right)\right]$-structure $\phi_{\eta}$ gives us a $\mu_{N}$-torsor $P_{\eta} \rightarrow E_{\eta}$, corresponding to $Q_{\eta}=\phi_{\eta}(1) \in \operatorname{Pic}_{E_{\eta} / K}^{0}(K) \cong E_{\eta}(K)$ of "exact order $N$ " in the sense of [Katz and Mazur 1985, §1.4]. $Q_{\eta}$ extends to a "possibly nonample $\left[\Gamma_{1}(N)\right]$-structure" on $\mathcal{E} / R$. Since the special fiber $\mathcal{E}_{s}$ is geometrically a Néron $N$-gon, after further base change on $R$ we may assume $\mathcal{E}_{s}^{\mathrm{sm}}[N] \cong \mu_{N} \times \mathbb{Z} /(N)$, so

$$
\mathcal{E}_{s}^{\mathrm{sm}}(k(s))[N] \cong \mu_{N}(k(s)) \times \mathbb{Z} /(N)=\{1\} \times \mathbb{Z} /(N) \cong \mathbb{Z} /(N) .
$$


Suppose $d \geq 1$ is minimal such that $d \cdot Q_{s}$ maps to 0 in $\mathbb{Z} /(N)$. Then we can choose $Q^{1}, Q^{2} \in \mathcal{E}^{\mathrm{sm}}(R)[N]$ such that:

- $Q=Q^{1}+Q^{2}$ in $\mathcal{E}^{\mathrm{sm}}(R)$,

- $Q^{1}$ has exact order $d$ in the abelian group $\mathcal{E}^{\mathrm{sm}}(R)[N]$, and the relative effective Cartier divisor

$$
\sum_{a=0}^{d-1}[a \cdot Q]
$$

in $\mathcal{E}^{\mathrm{sm}}$ is étale over $\operatorname{Spec}(R)$, and

- $Q_{s}^{2}$ maps to 0 in $\mathbb{Z} /(N)$ via the above isomorphism.

In the abelian group $\mathcal{E}^{\mathrm{sm}}(R)[N] \cong(\mathbb{Z} /(N))^{2}, Q^{2}$ has exact order $e$ for some $e \mid N$. Therefore $Q_{\eta}^{1}$ is a $\left[\Gamma_{1}(d)\right]$-structure on $E_{\eta}$, and $Q_{\eta}^{2}$ is a $\left[\Gamma_{1}(e)\right]$-structure on $E_{\eta}$. They correspond via Lemma 3.19 to the $\mu_{d}$-torsor

$$
P_{\eta}^{1}:=\underline{\operatorname{Spec}}_{E_{\eta}}\left(\bigoplus_{a=0}^{d-1} \mathscr{L}\left(\left(a \cdot Q_{\eta}^{1}\right)-\left(0_{E_{\eta}}\right)\right)\right)
$$

and the $\mu_{e}$-torsor

$$
P_{\eta}^{2}:=\underline{\operatorname{Spec}}_{E_{\eta}}\left(\bigoplus_{a=0}^{e-1} \mathscr{L}\left(\left(a \cdot Q_{\eta}^{2}\right)-\left(0_{E_{\eta}}\right)\right)\right),
$$

respectively, with the gradings determining the actions of $\mu_{d}$ and $\mu_{e}$. The $\mu_{N}$-torsor corresponding to $Q_{\eta}$ via Lemma 3.19 is

$$
P_{\eta}:=\underline{\operatorname{Spec}}_{E_{\eta}}\left(\bigoplus_{a=0}^{N-1} \mathscr{L}\left(\left(a \cdot Q_{\eta}\right)-\left(0_{E_{\eta}}\right)\right)\right) .
$$

The group law on $E_{\eta}$ tells us that

$$
\left(\left(Q_{\eta}^{1}\right)-\left(0_{E_{\eta}}\right)\right)+\left(\left(Q_{\eta}^{2}\right)-\left(0_{E_{\eta}}\right)\right) \sim\left(Q_{\eta}^{1}+Q_{\eta}^{2}\right)-\left(0_{E_{\eta}}\right)=\left(Q_{\eta}\right)-\left(0_{E_{\eta}}\right),
$$

so we conclude that

$$
P_{\eta}=\underline{\operatorname{Spec}}_{E_{\eta}}\left(\bigoplus_{a=0}^{N-1} \mathscr{L}\left(\left(a \cdot Q_{\eta}^{1}\right)-\left(0_{E_{\eta}}\right)\right) \otimes \mathscr{L}\left(\left(a \cdot Q_{\eta}^{2}\right)-\left(0_{E_{\eta}}\right)\right)\right),
$$

with the $\mu_{N}$-action induced by the grading.

Consider the $\mu_{d}$-torsor $P_{\eta}^{1} \rightarrow E_{\eta}$. As in Lemma 4.11, factoring the isogeny [ $\left.d\right]$ on $E_{\eta}$ as $E_{\eta} \rightarrow E_{\eta} /\left\langle Q_{\eta}^{1}\right\rangle \rightarrow E_{\eta}$, we have $P_{\eta}^{1}=E_{\eta} /\left\langle Q_{\eta}^{1}\right\rangle$ with $\mu_{d}$ acting on $P_{\eta}^{1}$ through the isomorphism with the group scheme $E_{\eta}[d] /\left\langle Q_{\eta}^{1}\right\rangle$ induced via

$$
E_{\eta}[d] /\left\langle Q_{\eta}^{1}\right\rangle \cong\left\{Q_{\eta}^{1}\right\} \times E_{\eta}[d] /\left\langle Q_{\eta}^{1}\right\rangle \hookrightarrow\left\langle Q_{\eta}^{1}\right\rangle \times E_{\eta}[d] /\left\langle Q_{\eta}^{1}\right\rangle \stackrel{e_{d}}{\rightarrow} \mu_{d} .
$$


Let $\varepsilon_{1} / R$ (possibly after base change on $R$ ) be a generalized elliptic curve extending $E_{\eta}$, whose closed fiber is geometrically a Néron $d$-gon, with $\mathcal{E}_{1}^{\mathrm{sm}}(R)[d] \cong E_{\eta}(K)[d]$.
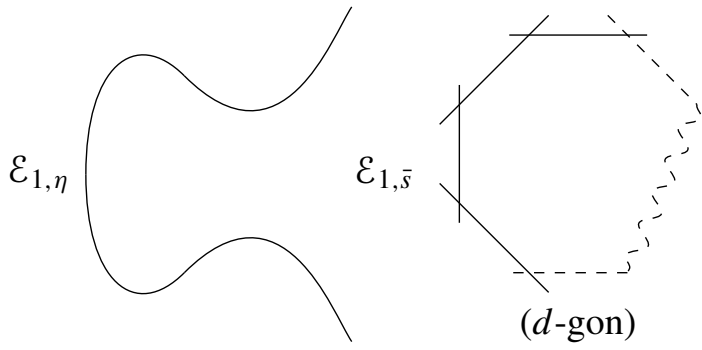

As in Lemma 4.11, after further base change on $R$ we may assume that the $e_{d}$-pairing on $E_{\eta}$ extends to a nondegenerate bilinear pairing of finite flat commutative group schemes over $R$

$$
e_{d}: \mathcal{E}_{1}^{\mathrm{sm}}[d] \times \mathcal{E}_{1}^{\mathrm{sm}}[d] \rightarrow \mu_{d} .
$$

$Q_{\eta}^{1}$ extends to a $\left[\Gamma_{1}(d)\right]$-structure $Q^{1}$ on $\mathcal{E}_{1} / R$, and the relative effective Cartier divisor

$$
D:=\sum_{a=0}^{d-1}\left[a \cdot Q^{1}\right]
$$

in $\mathcal{E}_{1}^{\mathrm{sm}}$ is étale over $R$, so via the same formula as above we see that the isomorphism $E_{\eta}[d] /\left\langle Q_{\eta}^{1}\right\rangle \cong \mu_{d}$ extends to a group scheme isomorphism $\mathcal{E}_{1}^{\mathrm{sm}}[d] /\left\langle Q^{1}\right\rangle \cong \mu_{d}$. This makes $P^{1}:=\left[\mathcal{E}_{1} /\left\langle Q^{1}\right\rangle\right]=\mathcal{E}_{1} /\left\langle Q_{1}\right\rangle$ a representable $\mu_{d}$-torsor over the twisted curve $\mathscr{C}:=\left[\mathcal{E} / \mathcal{E}^{\mathrm{sm}}[d]\right]$, extending the given $\mu_{d}$-torsor $P_{\eta}^{1} \rightarrow E_{\eta}$.

The special fiber $\mathscr{C}_{s}$ is geometrically a standard $\mu_{d}$-stacky Néron 1-gon, say with coarse space $\pi: \mathscr{C}_{s} \rightarrow C_{s} ;\left[\mathcal{E}_{1, s} / D_{s}\right]=\mathcal{E}_{1, s} / D_{s}$ is geometrically a (nonstacky) Néron 1-gon, and the quotient map $P_{s}^{1}:=\mathcal{E}_{1, s} / D_{s} \rightarrow \mathscr{C}_{s}$ extends the map $P_{s}^{1, \mathrm{sm}}=$ $\mathbb{G}_{m} \rightarrow \mathscr{C}_{s}^{\mathrm{sm}}=\mathbb{G}_{m}, x \mapsto x^{d}$.

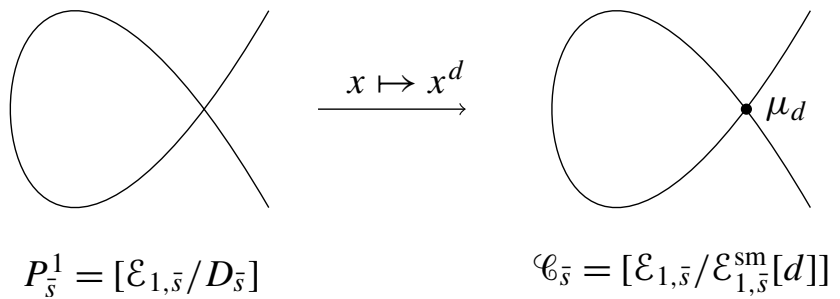

In particular, if $\bar{q} \rightarrow C_{s}$ is a geometric point mapping to the node of $C_{s}$, then $\mathscr{C} \times_{C} \bar{q} \cong\left(\mathscr{B} \mu_{d}\right)_{k(\bar{q})}$ and $P_{s}^{1} \times_{C} \bar{q}=\bar{q}$, so the resulting $\mu_{d}$-torsor over $\left(\mathscr{B} \mu_{d}\right)_{k(\bar{q})}$ corresponds to a generator of $H^{1}\left(\mathscr{B} \mu_{d}, \mu_{d}\right) \cong \mathbb{Z} /(d)$. Therefore, with respect to the decomposition

$$
\operatorname{Pic}_{C_{s} / k(s)}^{0} \cong \operatorname{Pic}_{C_{s} / k(s)}^{0} \times H^{0}\left(C_{s}, R^{1} \pi_{*} \mathbb{G}_{m}\right) \cong \mathbb{G}_{m} \times \mathbb{Z} /(d),
$$


the class of the $\mu_{d}$-torsor $P_{s}^{1} \rightarrow \mathscr{C}_{s}$ projects in the second factor to a generator of $\mathbb{Z} /(d)$. Therefore the group scheme homomorphism $\mathbb{Z} /(d) \rightarrow \mathrm{Pic}_{\mathscr{C} / R}^{0}$ defined by the $\mu_{d}$-torsor $P^{1}:=\left[\mathcal{E}_{1} / D\right]=\mathcal{E}_{1} / D$ over $\mathscr{C}$ is a $\left[\Gamma_{1}(d)\right]$-structure on the twisted curve $\mathscr{b} / R$.

Next consider the $\mu_{e}$-torsor $P_{\eta}^{2} \rightarrow E_{\eta}$. Let $\pi: \mathscr{C} \rightarrow C$ be the coarse space of the twisted curve $\mathscr{C} / R$ described above. By [Deligne and Rapoport 1973, IV.1.6], after further base change on $R$ we may assume that $C / R$ is a generalized elliptic curve, with structure extending that of $E_{\eta}$; note that $C_{\eta}=E_{\eta}$ and that $C_{s}$ is geometrically a Néron 1-gon.
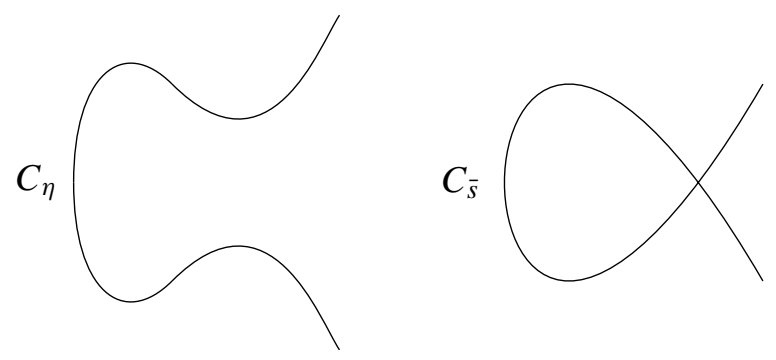

We may take the scheme-theoretic closure of the section $Q_{\eta}^{2} \in E_{\eta}(K)$ to get a unique section $Q^{2} \in C^{\mathrm{sm}}(R)$; necessarily $e \cdot Q^{2}=0_{C}$ since $e \cdot Q_{\eta}^{2}=0_{E_{\eta}}$. The isomorphisms

$$
\mathscr{L}\left(\left(a \cdot Q_{\eta}^{2}\right)-\left(0_{E_{\eta}}\right)\right) \otimes \mathscr{L}\left(\left(b \cdot Q_{\eta}^{2}\right)-\left(0_{E_{\eta}}\right)\right) \cong \mathscr{L}\left(\left((a+b) \cdot Q_{\eta}^{2}\right)-\left(0_{E_{\eta}}\right)\right)
$$

of line bundles on $E_{\eta}$ extend uniquely to isomorphisms

$$
\mathscr{L}\left(\left(a \cdot Q^{2}\right)-\left(0_{C}\right)\right) \otimes \mathscr{L}\left(\left(b \cdot Q^{2}\right)-\left(0_{C}\right)\right) \cong \mathscr{L}\left(\left((a+b) \cdot Q^{2}\right)-\left(0_{C}\right)\right)
$$

of line bundles on $C$; therefore the $\mu_{e}$-torsor

$$
P_{\eta}^{2}=\underline{\operatorname{Spec}}_{E_{\eta}}\left(\bigoplus_{a=0}^{e-1} \mathscr{L}\left(\left(a \cdot Q_{\eta}^{2}\right)-\left(0_{E_{\eta}}\right)\right)\right)
$$

extends uniquely to a $\mu_{e}$-torsor

$$
P^{2}:=\underline{\operatorname{Spec}}_{C}\left(\bigoplus_{a=0}^{e-1} \mathscr{L}\left(\left(a \cdot Q^{2}\right)-\left(0_{C}\right)\right)\right)
$$

over $C$, with the $\mu_{e}$-action induced by the grading. Since $\mathrm{Pic}_{C / R}^{0}$ has irreducible geometric fibers, this is a $\left[\Gamma_{1}(e)\right]$-structure on the generalized elliptic curve $C / R$. Pulling this back to $\mathscr{C}$ via the coarse moduli space map $\pi: \mathscr{C} \rightarrow C$, we get a $\mu_{e}$-torsor $\mathscr{P}^{2} \rightarrow \mathscr{C}$ extending $P_{\eta}^{2} \rightarrow E_{\eta}$, such that the corresponding map $\phi: \mathbb{Z} /(N) \rightarrow \operatorname{Pic}_{\mathscr{C} / R}^{0}$ lands in the identity component of every geometric fiber. 
Finally, we return to the $\mu_{N}$-torsor $P_{\eta} \rightarrow E_{\eta}$. Write the $\mu_{d}$-torsor $P^{1} \rightarrow \mathscr{C}$ as

$$
P^{1}=\underline{\operatorname{Spec}}_{\mathscr{C}}\left(\bigoplus_{a=0}^{d-1} \mathscr{L}_{a}\right) \text {, }
$$

so $\left.\mathscr{L}_{a}\right|_{\eta}=\mathscr{L}\left(\left(a \cdot Q_{\eta}^{1}\right)-\left(0_{E_{\eta}}\right)\right)$; write the $\mu_{e}$-torsor $P^{2} \rightarrow \mathscr{C}$ as

$$
P^{2}=\underline{\operatorname{Spec}}_{\mathscr{C}}\left(\bigoplus_{a=0}^{e-1} \mathscr{L}_{a}^{\prime}\right),
$$

so $\left.\mathscr{L}_{a}^{\prime}\right|_{\eta}=\mathscr{L}\left(\left(a \cdot Q_{\eta}^{2}\right)-\left(0_{E_{\eta}}\right)\right)$. Consider the $\mu_{N}$-torsor

$$
P:=\underline{\operatorname{Spec}}_{\mathscr{C}}\left(\bigoplus_{a=0}^{N-1} \mathscr{L}_{a \bmod d} \otimes \mathscr{L}_{a \bmod e}^{\prime}\right)
$$

over $\mathscr{b}$, with the $\mu_{N}$-action induced by the grading. Since

$$
\mathscr{L}\left(\left(a \cdot Q_{\eta}^{1}\right)-\left(0_{E_{\eta}}\right)\right) \otimes \mathscr{L}\left(\left(a \cdot Q_{\eta}^{2}\right)-\left(0_{E_{\eta}}\right)\right) \cong \mathscr{L}\left(\left(a \cdot Q_{\eta}\right)-\left(0_{E_{\eta}}\right)\right),
$$

we conclude that $P \rightarrow \mathscr{C}$ extends the original $\mu_{N}$-torsor $P_{\eta} \rightarrow E_{\eta}$. Furthermore, with respect to the decomposition

$$
\operatorname{Pic}_{\mathscr{C}_{s} / k(s)}^{0} \cong \operatorname{Pic}_{C_{s} / k(s)}^{0} \times H^{0}\left(C_{s}, \boldsymbol{R}^{1} \pi_{*} \mathbb{G}_{m}\right) \cong \mathbb{G}_{m} \times \mathbb{Z} /(d),
$$

the line bundle $\left.\mathscr{L}_{1}\right|_{s}$ projects to a generator of $\mathbb{Z} /(d)$, and the line bundle $\left.\mathscr{L}_{1}^{\prime}\right|_{s}$ projects to $0 \in \mathbb{Z} /(d)$; therefore the line bundle $\left.\left(\mathscr{L}_{1} \otimes \mathscr{L}_{1}^{\prime}\right)\right|_{s}$ projects to a generator of $\mathbb{Z} /(d)$, so the group scheme homomorphism $\mathbb{Z} /(N) \rightarrow \operatorname{Pic}_{\mathscr{C} / R}^{0}$ corresponding to the $\mu_{N}$-torsor $P \rightarrow \mathscr{C}$ is a $\left[\Gamma_{1}(N)\right]$-structure on $\mathscr{C} / R$, extending our original $\left[\Gamma_{1}(N)\right]$-structure $\phi_{\eta}: \mathbb{Z} /(N) \rightarrow \operatorname{Pic}_{\mathscr{C}_{\eta} / K}^{0}$.

This concludes the proof of our final lemma and thus of Theorem 4.6.

Reduction mod $\boldsymbol{p}$ of $\overline{\mathscr{K}}_{\mathbf{1 , 1}}^{\prime}\left(\mathscr{\Re} \boldsymbol{\mu}_{N}\right)$. The analysis of $\mathcal{H}_{1}(N)$ above immediately generalizes to the compactified case. Recall from Notation 3.8 that $\overline{\mathscr{K}}_{1,1}^{\prime}\left(\mathscr{B} \mu_{N}\right)$ $\subset \overline{\mathscr{K}}_{1,1}\left(\mathscr{B} \mu_{N}\right)$ denotes the closed substack classifying rigidified twisted stable $\mu_{N}$-covers of twisted curves with nonstacky marking; $\overline{\mathscr{K}}_{1,1}^{\prime}\left(\Re \mu_{N}\right)$ is the closure of $\overline{\mathscr{K}}_{1,1}^{\circ}\left(\mathscr{B} \mu_{N}\right) \simeq \mathcal{H}_{1}(N)$ in $\overline{\mathscr{K}}_{1,1}\left(\mathscr{\Re} \mu_{N}\right)$ by Lemma 3.21 .

We have a natural closed immersion

$$
\iota^{(d)}: X_{1}^{\mathrm{tw}}(d) \hookrightarrow \overline{\mathscr{K}}_{1,1}^{\prime}\left(\mathscr{B} \mu_{N}\right)
$$

for each $d$ dividing $N$, precomposing a map $\mathbb{Z} /(d) \rightarrow \operatorname{Pic}_{\mathscr{C} / S}^{0}$ with the canonical projection $\mathbb{Z} /(N) \rightarrow \mathbb{Z} /(d)$. The resulting map

$$
\bigsqcup_{d \mid N} X_{1}^{\mathrm{tw}}(d) \rightarrow \overline{\mathscr{K}}_{1,1}^{\prime}\left(\mathscr{B} \mu_{N}\right)
$$

is an isomorphism over $S[1 / N]$. 
Definition 4.13. Let $p$ be prime and $S \in \mathrm{Sch} / \mathbb{F}_{p}$. Let $\mathscr{C} / S$ be a 1 -marked genus-1 twisted stable curve with no stacky structure at its marking. Let $n \geq 1$ and $a, b \geq 0$ with $a+b=n$. A $\left[\Gamma_{1}\left(p^{n}\right)\right]$ - $(a, b)$-cyclic structure on $\mathscr{C}$ is a $\left[\Gamma_{1}\left(p^{n}\right)\right]$-structure $\phi: \mathbb{Z} /\left(p^{n}\right) \rightarrow \mathrm{Pic}_{\mathscr{C} / S}^{0}$, such that:

- if $S_{1} \subset S$ is the maximal Zariski open subset such that $\mathscr{C}_{S_{1}} \rightarrow S_{1}$ is smooth, $\phi_{S_{1}}: \mathbb{Z} /\left(p^{n}\right) \rightarrow \mathscr{C}_{S_{1}}$ is a $\left[\Gamma_{1}\left(p^{n}\right)\right]-(a, b)$-cyclic structure in the sense of [Katz and Mazur 1985], and

- if $S_{2} \subset S$ is the complement of the supersingular locus of $\mathscr{b} \rightarrow S$, then the relative effective Cartier divisor

$$
D:=\sum_{m=1}^{p^{b}}[\phi(m)]
$$

in $\operatorname{Pic}_{\mathscr{C}_{S_{2} / S_{2}}}^{0}$ is a subgroup scheme of $\operatorname{Pic}_{\mathscr{C}_{S_{2}} / S_{2}}^{0}$ which is étale over $S_{2}$.

Over the base scheme $S \in \mathrm{Sch} / \mathbb{F}_{p}$, we define $X_{1}^{\mathrm{tw}}\left(p^{n}\right)^{(a, b)} \subset X_{1}^{\mathrm{tw}}\left(p^{n}\right)$ to be the closed substack associating to $T / S$ the groupoid of pairs $(\mathscr{C}, \phi)$, where $\mathscr{C} / S$ is a 1-marked genus-1 twisted stable curve with nonstacky marking, and $\phi$ is a $\left[\Gamma_{1}\left(p^{n}\right)\right]$ - $(a, b)$-cyclic structure on $\mathscr{b}$.

If $N=p^{n} N^{\prime}$ with $\left(N^{\prime}, p\right)=1$, we define

$$
X_{1}^{\mathrm{tw}}(N)^{(a, b)}:=X_{1}^{\mathrm{tw}}\left(N^{\prime}\right) \times_{\bar{M}_{1,1}} X_{1}^{\mathrm{tw}}\left(p^{n}\right)^{(a, b)} .
$$

The same argument as that used to prove Lemma 2.11 immediately gives us:

Lemma 4.14. Let $\mathscr{G} / S / \mathbb{F}_{p}$ be a 1-marked genus-1 twisted stable curve with nonstacky marking, and let $\phi: \mathbb{Z} /\left(p^{n}\right) \rightarrow \operatorname{Pic}_{\mathscr{C} / S}^{0}$ be $a\left[\Gamma_{1}\left(p^{n}\right)\right]-(a, b)$-cyclic structure on $\mathscr{C}$. Then for the canonical projection $\pi: \mathbb{Z} /\left(p^{n+1}\right) \rightarrow \mathbb{Z} /\left(p^{n}\right)$, the composite $\phi \circ \pi: \mathbb{Z} /\left(p^{n+1}\right) \rightarrow \operatorname{Pic}_{\mathscr{C} / S}^{0}$ is a $\left[\Gamma_{1}\left(p^{n+1}\right)\right]$-structure on $\mathscr{C}$, and is $\left[\Gamma_{1}\left(p^{n+1}\right)\right]-(a+1, b)-$ cyclic.

$$
\text { If } N=p^{n} N^{\prime} \text { with }\left(N^{\prime}, p\right)=1 \text {, for any } r \mid N^{\prime} \text { write } \overline{\mathscr{K}}_{1,1}^{\prime}\left(\mathscr{\Re} \mu_{N}\right)^{r} \subset \overline{\mathscr{K}}_{1,1}^{\prime}\left(\mathscr{\Re} \mu_{N}\right)
$$
for the union of the components $X_{1}^{\mathrm{tw}}\left(p^{m} r\right)$ over $0 \leq m \leq n$.

Corollary 4.15. Let $k$ be a perfect field of characteristic $p$, and let $N=p^{n} N^{\prime}$ where $\left(N^{\prime}, p\right)=1$. For any $r \mid N^{\prime}, \overline{\mathscr{K}}_{1,1}^{\prime}\left(\mathscr{B} \mu_{N}\right)_{k}^{r}$ is the disjoint union, with crossings at the supersingular points, of components $z_{b}^{r}$ for $0 \leq b \leq n$, where

$$
z_{b}^{r}=\bigcup_{b \leq m \leq n} X_{1}^{\mathrm{tw}}\left(p^{m} r\right)_{k}^{(m-b, b)}
$$

identifying each $X_{1}^{\mathrm{tw}}\left(p^{m} r\right)_{k}^{(m-b, b)}$ with a closed substack of $\overline{\mathscr{K}}_{1,1}^{\prime}\left(\mathscr{B} \mu_{N}\right)_{k}$ via $\iota^{\left(p^{m} r\right)}$. Each substack $X_{1}^{\mathrm{tw}}\left(p^{m} r\right)_{k}^{(m-b, b)}$ is "set-theoretically identified with $Z_{b}^{r}$ " in the sense 
that $\left(\mathcal{Z}_{b}^{r}\right)_{\text {red }}=X_{1}\left(p^{m} r\right)_{k, \text { red }}^{(m-b, b)}$ as substacks of $\overline{\mathscr{K}}_{1,1}^{\prime}\left(\mathscr{B} \mu_{N}\right)_{k, \text { red. }} \overline{\mathscr{K}}_{1,1}^{\prime}\left(\mathscr{B} \mu_{N}\right)_{k}$ is the disjoint union of the open and closed substacks

$$
\left\{\overline{\mathscr{K}}_{1,1}^{\prime}\left(\mathscr{\Re} \mu_{N}\right)_{k}^{r}\right\}_{r \mid N^{\prime}}
$$

Strictly speaking, to apply the crossings theorem (Theorem 2.7) to get the above corollary, we need to know that the morphism $\overline{\mathscr{K}}_{1,1}^{\prime}\left(\mathscr{\Re} \mu_{N}\right) \rightarrow \bar{M}_{1,1}$ is finite. This follows from Corollary 6.3.

The picture in the case $N=p^{n}$ is essentially the same as the picture for $\overline{\mathscr{K}}_{1,1}^{\circ}\left(\mathscr{B} \mu_{p^{n}}\right)_{k}$ (as discussed after Proposition 4.1), except now each component is proper:

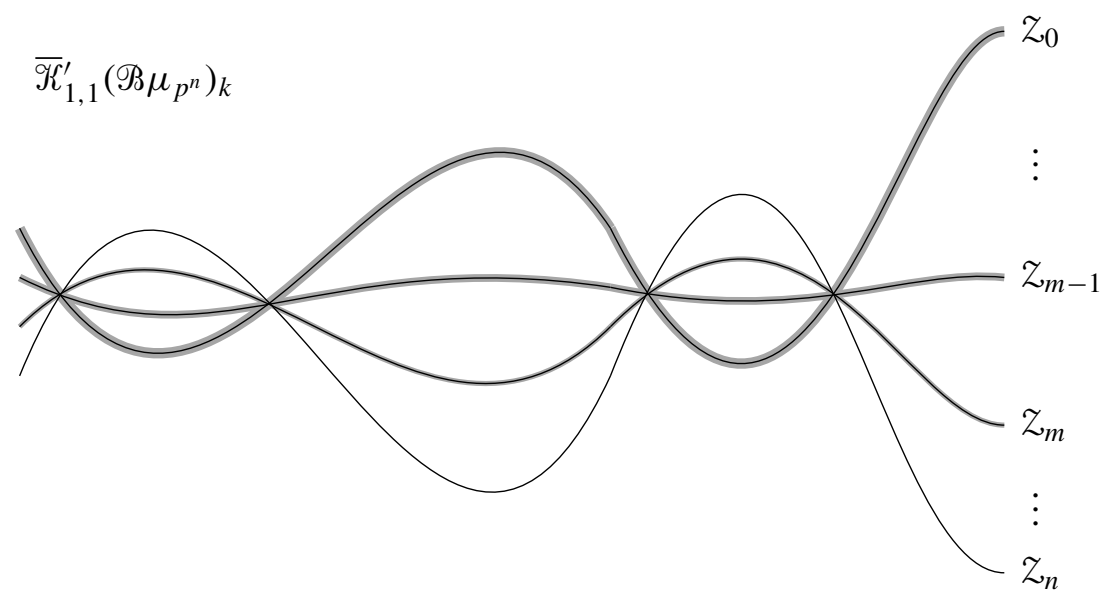

5. Moduli of elliptic curves in $\overline{\mathscr{K}}_{1,1}\left(\mathscr{\Re}_{N}^{2}\right)$

Reduction mod $\boldsymbol{p}$ of $\mathcal{H}(N)$. Next we turn our attention to $\overline{\mathscr{K}}_{1,1}\left(\mathscr{B}_{N}^{2}\right)$, working as before over an arbitrary base scheme $S$. Recall that by Corollary 3.20, the open substack $\overline{\mathscr{K}}_{1,1}^{\circ}\left(\mathscr{B} \mu_{N}^{2}\right)$ classifying rigidified twisted $\mu_{N}^{2}$-covers of smooth elliptic curves is naturally equivalent to the stack $\mathcal{H}(N)$ associating to a scheme $T / S$ the groupoid of pairs $(E, \phi)$ where $E / T$ is an elliptic curve and $\phi:(\mathbb{Z} /(N))^{2} \rightarrow E[N]$ is a homomorphism of group schemes over $T$.

For any subgroup $K \leq(\mathbb{Z} /(N))^{2}$ with corresponding quotient $G_{K}=(\mathbb{Z} /(N))^{2} / K$ of $(\mathbb{Z} /(N))^{2}$, recall (Definition 2.16) that $y_{K}$ denotes the moduli stack associating to a scheme $T / S$ the groupoid of pairs $(E, \psi)$, where $E / T$ is an elliptic curve and $\psi: G_{K} \rightarrow E$ is a $G_{K}$-structure (in the sense of [Katz and Mazur 1985, §1.5]). So, for example, if $G_{K} \cong \mathbb{Z} /(d)$ for some $d \mid N, y_{K}$ is isomorphic to the stack $y_{1}(d)$, and if $G_{K} \cong(\mathbb{Z} /(d))^{2}$ then $y_{K}$ is isomorphic to the stack $y(d)$ classifying (not necessarily symplectic) $[\Gamma(d)]$-structures on elliptic curves. For every such $K$, we 
have a closed immersion

$$
\iota^{K}: y_{K} \hookrightarrow \mathcal{H}(N),
$$

given by precomposing a $G_{K}$-structure $\phi: G_{K} \rightarrow E[N]$ with the canonical projection $(\mathbb{Z} /(N))^{2} \rightarrow G_{K}$. Together, these give a proper surjection

$$
\bigsqcup_{K \leq(\mathbb{Z} /(N))^{2}} y_{K} \rightarrow \mathcal{H}(N)
$$

which is an isomorphism over $S[1 / N]$.

But in characteristics dividing $N$ this is not an isomorphism. First we consider the case where $N=p^{n}$ for some prime $p$. Any quotient $G_{K}=\left(\mathbb{Z} /\left(p^{n}\right)\right)^{2} / K$ is isomorphic as an abelian group to $\mathbb{Z} /\left(p^{m}\right) \times \mathbb{Z} /\left(p^{l}\right)$ for some $l \leq m \leq n$. The corresponding moduli stack $y_{K}$ classifies $G_{K}$-structures on elliptic curves, and we saw in Theorem 2.17 that over a perfect field $k$ of characteristic $p, y_{K, k}$ is the disjoint union, with crossings at the supersingular points, of substacks $y_{K, k}^{H}$ indexed by the set

$$
L_{K}:=\left\{H \leq G_{K} \mid H \text { and } G_{K} / H \text { are both cyclic }\right\} .
$$

The component $y_{K, k}^{H}$ classifies $G_{K}$-structures of component label $H$.

Now consider two subgroups $K^{\prime} \leq K \leq\left(\mathbb{Z} /\left(p^{n}\right)\right)^{2}$, and write $\pi: G_{K^{\prime}} \rightarrow G_{K}$ for the canonical surjection. If $\phi: G_{K} \rightarrow E\left[p^{n}\right]$ is a $G_{K}$-structure on an ordinary elliptic curve $E / T / k$, then $\phi \circ \pi$ may or may not be a $G_{K^{\prime}}$-structure on $E$. Indeed, we saw in Lemma 2.18 that $\phi \circ \pi$ is a $G_{K^{\prime}}$ structure if and only if $\pi^{-1}(H) \in L_{K^{\prime}}$, that is, if and only if $\pi^{-1}(H) \subseteq G_{K^{\prime}}$ is cyclic.

Consider the set $\left\{(K, H) \mid K \leq\left(\mathbb{Z} /\left(p^{n}\right)\right)^{2}, H \in L_{K}\right\}$. Let $\sim$ be the equivalence relation on this set generated by requiring that $(K, H) \sim\left(K^{\prime}, H^{\prime}\right)$ if $K^{\prime} \leq K$ and $H$ and $H^{\prime}$ are as above, and let $\Lambda=\{(K, H)\} / \sim$.

Proposition 5.1. Let $k$ be a perfect field of characteristic $p . \mathcal{H}\left(p^{n}\right)_{k}$ is the disjoint union, with crossings at the supersingular points, of components $\mathcal{H}\left(p^{n}\right)_{k}^{\lambda}$ for $\lambda \in \Lambda$, where

$$
\mathcal{H}\left(p^{n}\right)_{k}^{\lambda}:=\bigcup_{[(K, H)]=\lambda} y_{K, k}^{H}
$$

identifying each $y_{K, k}^{H}$ with a closed substack of $\mathcal{H}\left(p^{n}\right)_{k}^{\lambda}$ via $\iota^{K}$. Each $y_{K, k}^{H}$ is "settheoretically identified with $\mathcal{H}\left(p^{n}\right)_{k}^{\lambda}$ " in the sense that $\left(\mathcal{H}\left(p^{n}\right)_{k}^{\lambda}\right)_{\mathrm{red}}=\mathrm{y}_{K, k \text {, red }}^{H}$ as substacks of $\mathcal{H}\left(p^{n}\right)_{k, \text { red }}$ for all $(K, H)$ with $[(K, H)]=\lambda \in \Lambda$.

If $N=p^{n} N^{\prime}$ with $\left(p, N^{\prime}\right)=1$, for any $A \leq(\mathbb{Z} /(N))^{2}$ of order prime to $p$, let $\mathcal{H}(N)_{k}^{A} \subset \mathcal{H}(N)_{k}$ be the union of the substacks $y_{K, k}$ for $A \leq K \leq(\mathbb{Z} /(N))^{2}$ with $(K: A)$ a power of $p$. Then similarly $\mathcal{H}(N)_{k}^{A}$ is the disjoint union, with crossings at the supersingular points, of components $\mathcal{H}(N)_{k}^{A, \lambda}$ for $\lambda \in \Lambda$, and $\mathcal{H}(N)_{k}$ is the 
disjoint union of the open and closed substacks $\mathcal{H}(N)_{k}^{A}$ for $A \leq(\mathbb{Z} /(N))^{2}$ of order prime to $p$.

As in the case of $\mathcal{H}_{1}\left(p^{n}\right)$, the reduction mod $p$ of $\mathcal{H}\left(p^{n}\right)$ has an appealing geometric description. To keep our pictures from getting unreasonably large, we restrict our attention to the case $n=1$. The group $K:=(\mathbb{Z} /(p))^{2}$ has $p+3$ subgroups, namely the entire group $K, K_{0}:=0$, and $p+1$ subgroups $K_{1}, \ldots, K_{p+1}$ isomorphic to $\mathbb{Z} /(p)$. The corresponding moduli stacks are

$$
\begin{aligned}
& y_{K}=y(1), \\
& y_{K_{0}}=y(p), \\
& y_{K_{i}} \cong y_{1}(p) \quad \text { for } i=1, \ldots, p+1,
\end{aligned}
$$

so we see that over $\mathbb{Z}[1 / p], \mathcal{H}(p)$ is the disjoint union of $y(1), y(p)$, and $p+1$ copies of $y_{1}(p)$.

By definition we have

$$
\begin{aligned}
L_{K} & =\{0\}, \\
L_{K_{0}} & =\left\{K_{1}, \ldots, K_{p+1}\right\}, \\
L_{K_{i}} & =\left\{G_{K_{i}}, 0\right\} \quad \text { for } i=1, \ldots, p+1,
\end{aligned}
$$

where as usual $G_{K_{i}}=(\mathbb{Z} /(p))^{2} / K_{i}$. The set of labels $\Lambda$ is built by putting an equivalence relation on the set consisting of the following pairs:

$$
\begin{aligned}
(K, 0), & \\
\left(K_{0}, K_{i}\right) & \text { for } i=1, \ldots, p+1, \\
\left(K_{i}, G_{K_{i}}\right) & \text { for } i=1, \ldots, p+1, \\
\left(K_{i}, 0\right) & \text { for } i=1, \ldots, p+1 .
\end{aligned}
$$

By definition, working over a perfect field $k$ of characteristic $p$, the pair $(K, 0)$ corresponds to $\mathrm{y}(1)_{k}$; the pair $\left(K_{0}, K_{i}\right)$ corresponds to the component $y(p)_{k}^{K_{i}}$ of $y(p)_{k}$; the pair $\left(K_{i}, G_{K_{i}}\right)$ corresponds to the component $y_{1}(p)_{k}^{(1,0)}$ in $y_{K_{i}, k} \cong$ $y_{1}(p)_{k}$; and the pair $\left(K_{i}, 0\right)$ corresponds to the component $y_{1}(p)_{k}^{(0,1)}$ in $y_{K_{i}, k} \cong$ $y_{1}(p)_{k}$. Unwinding the definition, we see that the equivalence relation defining $\Lambda$ just says that $(K, 0) \sim\left(K_{i}, G_{K_{i}}\right)$ for $i=1, \ldots, p+1$, and that $\left(K_{0}, K_{i}\right) \sim\left(K_{i}, 0\right)$ for $i=1, \ldots, p+1$. Thus the set of labels is $\Lambda=\left\{\lambda_{0}, \lambda_{1}, \ldots, \lambda_{p+1}\right\}$, where

$$
\begin{aligned}
& \lambda_{0}=[(K, 0)]=\left[\left(K_{i}, G_{K_{i}}\right)\right] \text { for } i=1, \ldots, p+1 \text {, } \\
& \lambda_{i}=\left[\left(K_{0}, K_{i}\right)\right]=\left[\left(K_{i}, 0\right)\right] \quad \text { for } i=1, \ldots, p+1 .
\end{aligned}
$$

Visualize $\mathcal{H}(p)_{k, \text { red }}$ as follows: 


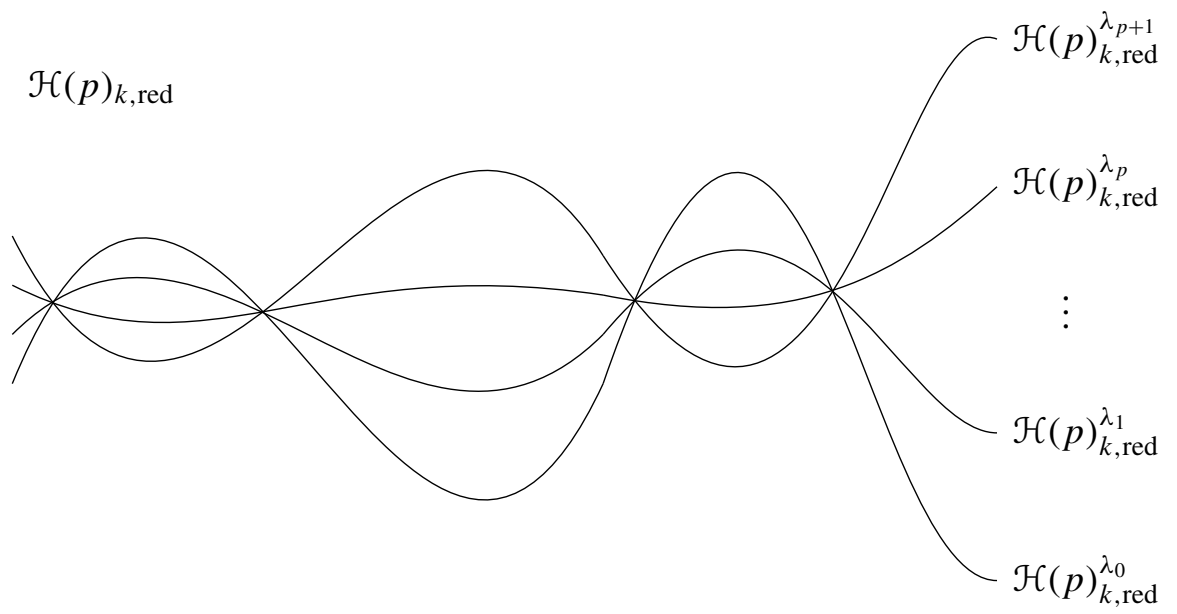

The component $\mathcal{H}(p)_{k}^{\lambda_{0}}$ is "set-theoretically identified" with the component $y(1)_{k}$ and with the component $y_{1}(p)_{k}^{(1,0)}$ in each copy of $y_{1}(p)_{k}$; each of these contributes additional nilpotent structure to the component $\mathcal{H}(p)_{k}^{\lambda_{0}}$ (each $y_{1}(p)_{k}^{(1,0)}$ has length $p-1$ over $\mathcal{H}(p)_{k \text {,red }}^{\lambda_{0}}=y(1)_{k}$, so $\mathcal{H}(p)_{k}^{\lambda_{0}}$ has length $(p+1)(p-1)+1=p^{2}$ over $\left.\mathcal{H}(p)_{k, \text { red }}^{\lambda_{0}}=y(1)_{k}\right)$.

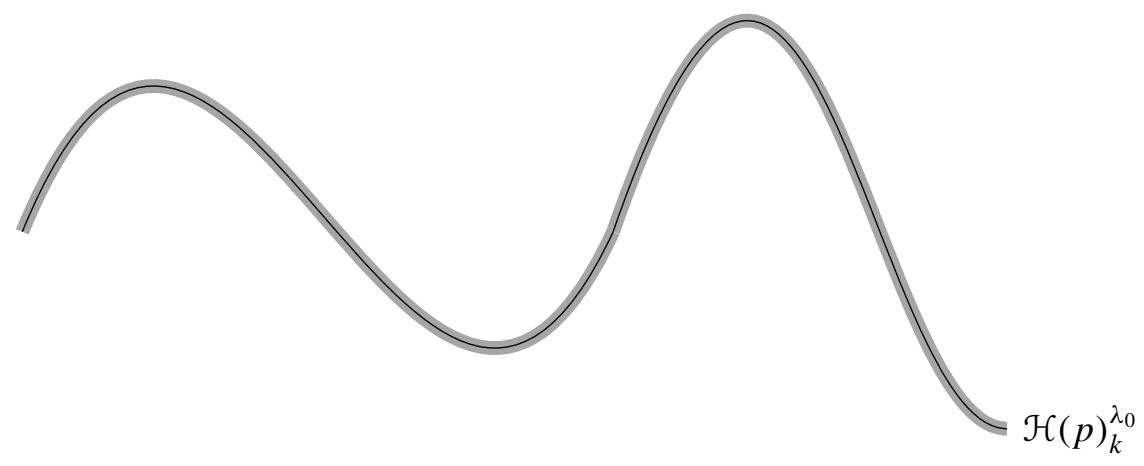

For $i=1, \ldots, p+1$, the component $\mathcal{H}(p)_{k}^{\lambda_{i}}$ is "set-theoretically identified" with the component $y(p)_{k}^{K_{i}}$ of $y(p)_{k}$, contributing nilpotent structure to $\mathcal{H}(p)_{k}^{\lambda_{i}}$ (each $y(p)_{k}^{K_{i}}$ has length $p-1$ over $\left.\mathcal{H}(p)_{k, \text { red }}^{\lambda_{i}}\right)$. The component $\mathcal{H}(p)_{k}^{\lambda_{i}}$ is also "settheoretically identified" with the component $y_{1}(p)_{k}^{(0,1)}$ of $y_{K_{i}, k} \cong y_{1}(p)_{k}$; each of these is reduced, adding 1 to the length of the component $\mathcal{H}(p)_{k}^{\lambda_{i}}$ over $\mathcal{H}(p)_{k \text {,red }}^{\lambda_{i}}$. Thus $\mathcal{H}(p)_{k}^{\lambda_{i}}$ has length $p$ over $\mathcal{H}(p)_{k, \text { red }}^{\lambda_{i}}$, which is isomorphic to $y_{1}(p)_{k}^{(0,1)}$ and hence has degree $p^{2}-p$ over $y(1)_{k}$ (see [Katz and Mazur 1985, 13.5.6]). The result is that the component $\mathcal{H}(p)_{k}^{\lambda_{i}}$ has length $p$ over the underlying reduced stack $y_{1}(p)_{k}^{(0,1)}$, which has degree $p^{2}-p$ over $y(1)_{k}$ : 


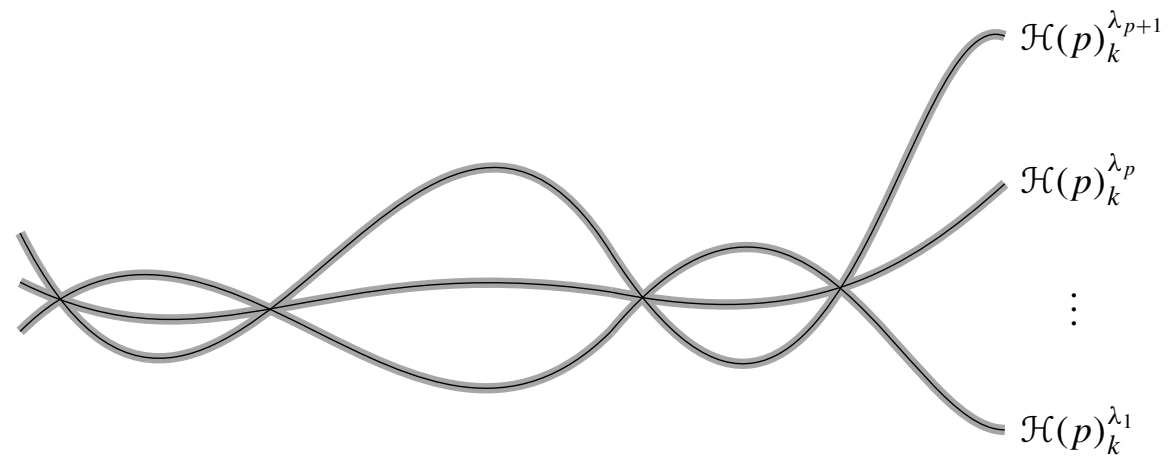

This gives us the following picture of $\mathcal{H}(p)_{k}$ :

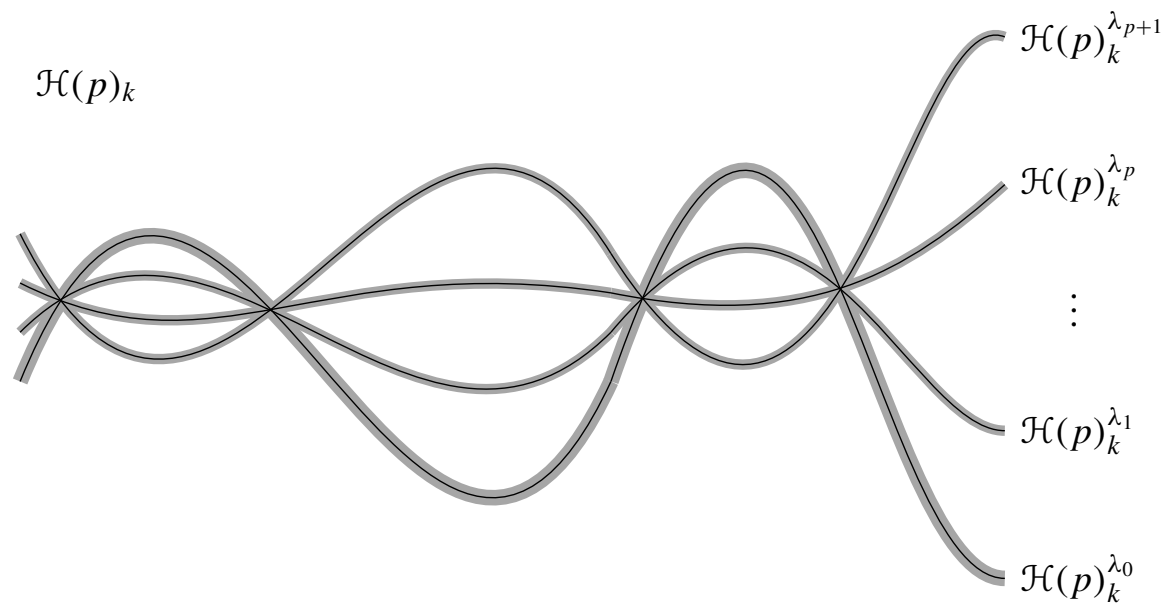

Note that adding up the lengths calculated in the course of the above construction, we recover the fact that the stack $\mathcal{H}(p)$ has length $p^{4}$ over $y(1)=M_{1,1}$.

Closure of $y(N)$ in $\overline{\mathscr{K}}_{1,1}\left(\mathscr{B} \mu_{N}^{2}\right)$.

Definition 5.2. Let $\mathscr{b} / S$ be a 1 -marked genus- 1 twisted stable curve over a scheme $S$, with no stacky structure at its marking. A $[\Gamma(N)]$-structure on $\mathscr{C}$ is a group scheme homomorphism $\phi:(\mathbb{Z} /(N))^{2} \rightarrow \mathrm{Pic}_{\mathscr{C} / S}^{0}$ such that:

- the relative effective Cartier divisor

$$
D:=\sum_{a \in(\mathbb{Z} /(N))^{2}}[\phi(a)]
$$

in $\mathrm{Pic}_{\mathscr{C} / S}^{0}$ is an $N$-torsion subgroup scheme, hence $D=\operatorname{Pic}_{\mathscr{C} / S}^{0}[N]$, and

- for every geometric point $\bar{p} \rightarrow S, D_{\bar{p}}$ meets every irreducible component of $\left(\mathrm{Pic}_{\mathscr{C} / S}^{0}\right)_{\bar{p}}=\operatorname{Pic}_{\mathscr{C}_{\bar{p}} / k(\bar{p})}^{0}$. 
We write $X^{\mathrm{tw}}(N)$ for the substack of $\overline{\mathscr{K}}_{1,1}\left(\mathscr{B} \mu_{N}^{2}\right)$ associating to $T / S$ the groupoid of pairs $(\mathscr{C}, \phi)$, where $\mathscr{C} / S$ is a 1 -marked genus-1 twisted stable curve with nonstacky marking, and $\phi$ is a $[\Gamma(N)]$-structure on $\mathscr{b}$.

If $\mathscr{b} / S$ is a twisted curve admitting a $[\Gamma(N)]$-structure and $\bar{p} \rightarrow S$ is a geometric point such that $C_{\bar{p}}$ is singular, then necessarily

$$
\operatorname{Pic}_{\mathscr{C}_{\bar{p}} / k(\bar{p})}^{0} \cong \mathbb{G}_{m} \times \mathbb{Z} /(N),
$$

so by Lemma $3.17 \mathscr{C}_{\bar{p}}$ is a standard $\mu_{N}$-stacky Néron 1-gon over $k(\bar{p})$, as in Example 3.4.

Applying the methods of our study of $X_{1}^{\mathrm{tw}}(N)$ to the stack $X^{\mathrm{tw}}(N)$, we have:

Theorem 5.3. Let $S$ be a scheme and let $X^{\mathrm{tw}}(N)$ be the stack over $S$ classifying $[\Gamma(N)]$-structures on 1-marked genus-1 twisted stable curves with nonstacky marking. Then $X^{\mathrm{tw}}(N)$ is a closed substack of $\overline{\mathscr{K}}_{1,1}\left(\mathscr{B} \mu_{N}^{2}\right)$, which contains $y(N)$ as an open dense substack.

In particular $X^{\mathrm{tw}}(N)$ is flat over $S$ with local complete intersection fibers, and is proper and quasifinite over $\bar{M}_{1,1}$.

Remark 5.4. In [Petersen 2012] a direct proof is given that over $\mathbb{Z}[1 / N], X^{\mathrm{tw}}(N)$ agrees with the stack $X(N)$ classifying [ $[(N)]$-structures on generalized elliptic curves; in this case a $\mu_{N}^{2}$-torsor over a 1-marked genus-1 twisted stable curve with nonstacky marking is in fact a generalized elliptic curve, and it is this observation that gives the desired equivalence. This argument does not generalize to characteristics dividing $N$, because, for example, if $N=p^{n}$ then the Néron $N$-gon in characteristic $p$ (which is a generalized elliptic curve admitting various $[\Gamma(N)]$-structures) cannot be realized as a $\mu_{N}^{2}$-torsor over a 1-marked genus-1 twisted stable curve.

Proof of Theorem 5.3. This is proved in exactly the same manner as Theorem 4.6. An immediate consequence of Lemma 3.22 is that if $E / K$ is an elliptic curve over a field $K$ in which $N$ is invertible, and $\left(Q_{1}, Q_{2}\right)$ is a $[\Gamma(N)]$-structure on $E$, then the $\mu_{N}^{2}$-torsor

$$
P=\underline{\operatorname{Spec}_{E}}\left(\bigoplus_{a, b \in \mathbb{Z} /(N)} \mathscr{L}\left(\left(a \cdot Q_{1}+b \cdot Q_{2}\right)-\left(0_{E}\right)\right)\right)
$$

over $E$ as in Corollary 3.20 may be identified with $E$ itself, with the quotient map $P \rightarrow E$ corresponding to the isogeny $[N]: E \rightarrow E$. We immediately deduce via the methods of Theorem 4.6 that over an algebraically closed field $k$ in which $N$ is invertible, the $\mu_{N}^{2}$-torsor obtained when we pass to the cusp of $\bar{M}_{1,1}$ is a Néron $N$-gon $P$ over a standard $\mu_{N}$-stacky Néron 1 -gon $\mathscr{C}$, with the $\mu_{N}^{2}$-action on $P$ induced by some choice of isomorphism $P^{\mathrm{sm}}[N] \cong \mu_{N}^{2}$ : 

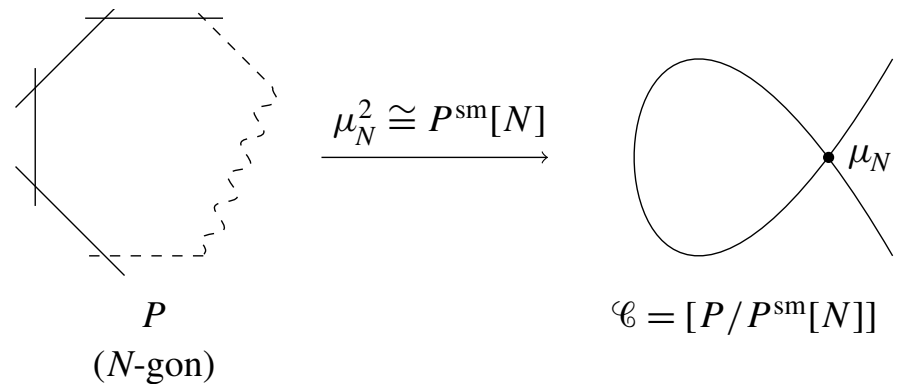

And in the case of $N=p^{n}$, over an algebraically closed $k$ field of characteristic $p$, the $\mu_{p^{n}}^{2}$-torsor $P$ obtained in passing to the cusp of $\bar{M}_{1,1}$ may be realized as a trivial

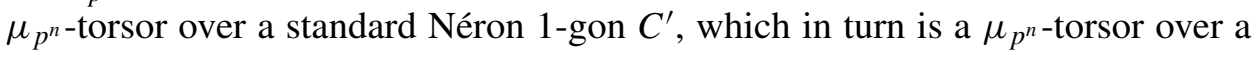

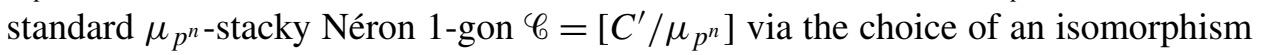
$\left(C^{\prime}\right)^{\mathrm{sm}}\left[p^{n}\right] \cong \mu_{p^{n}}$ :

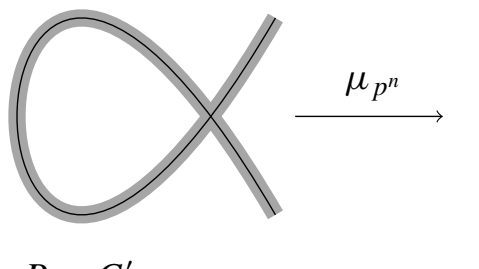

$$
P=C^{\prime} \times \mu_{p^{n}}
$$

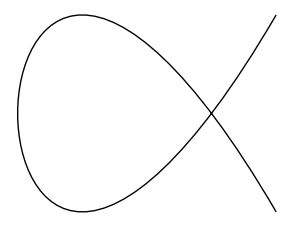

$C^{\prime}$

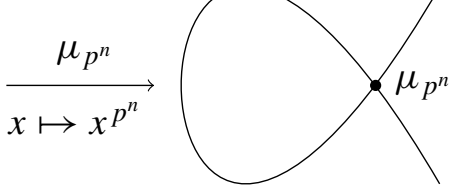

$\mathscr{b}$

In both of the above cases, it is immediately verified that for each of these $\mu_{N}^{2}$-torsors, the corresponding group scheme homomorphism $(\mathbb{Z} /(N))^{2} \rightarrow \operatorname{Pic}_{\mathscr{C} / k}^{0} \cong \mathbb{G}_{m} \times \mathbb{Z} /(N)$ is a $[\Gamma(N)]$-structure on the standard $\mu_{N}$-stacky Néron 1-gon $\mathscr{C}$ in the sense of Definition 5.2, giving the valuative criterion of properness for $X^{\mathrm{tw}}(N)$, hence Theorem 5.3.

Reduction mod $\boldsymbol{p}$ of $\overline{\mathscr{K}}_{\mathbf{1}, \mathbf{1}}^{\prime}\left(\mathscr{B} \boldsymbol{\mu}_{N}^{\mathbf{2}}\right)$. Recall that $\overline{\mathscr{K}}_{1,1}^{\prime}\left(\mathscr{B} \mu_{N}^{2}\right) \subset \overline{\mathscr{K}}_{1,1}\left(\mathscr{B} \mu_{N}^{2}\right)$ is the closed substack classifying rigidified twisted stable $\mu_{N}^{2}$-covers of twisted curves with nonstacky marking; so $\overline{\mathscr{K}}_{1,1}^{\prime}\left(\mathscr{B} \mu_{N}^{2}\right)$ is the closure of

$$
\overline{\mathscr{K}}_{1,1}^{\circ}\left(\mathscr{B} \mu_{N}^{2}\right) \simeq \mathcal{H}(N)
$$

in $\overline{\mathscr{K}}_{1,1}\left(\mathscr{B} \mu_{N}^{2}\right)$.

Definition 5.5. Let $\mathscr{C} / S$ be a 1-marked genus-1 twisted stable curve with nonstacky marking, and let $G$ be a 2-generated finite abelian group, say $G \cong \mathbb{Z} /\left(n_{1}\right) \times \mathbb{Z} /\left(n_{2}\right)$, $n_{1} \geq n_{2}$. A $G$-structure on $\mathscr{C}$ is a homomorphism $\phi: G \rightarrow \operatorname{Pic}_{\mathscr{C} / S}^{0}$ of group schemes over $S$ such that:

- the relative effective Cartier divisor

$$
D:=\sum_{a \in G}[\phi(a)]
$$


in $\operatorname{Pic}_{\mathscr{C} / S}^{0}$ is an $n_{1}$-torsion subgroup scheme and

- for every geometric point $\bar{p} \rightarrow S, D_{\bar{p}}$ meets every irreducible component of $\left(\operatorname{Pic}_{C_{C} / S}^{0}\right)_{\bar{p}}=\operatorname{Pic}_{\mathscr{C}_{\bar{p}} / k(\bar{p})}^{0}$.

For any subgroup $K \leq(\mathbb{Z} /(N))^{2}$ with corresponding quotient $G_{K}=(\mathbb{Z} /(N))^{2} / K$ of $(\mathbb{Z} /(N))^{2}$, we write $X_{K}^{\text {tw }}$ for the moduli stack over $S$ associating to a scheme $T / S$ the groupoid of pairs $(\mathscr{C}, \psi)$, where $\mathscr{C} / T$ is a 1 -marked genus- 1 twisted stable curve with nonstacky marking, and $\psi: G_{K} \rightarrow \operatorname{Pic}_{\mathscr{C} / T}^{0}$ is a $G_{K}$-structure on $\operatorname{Pic}_{\mathscr{C} / T}^{0}$.

For a twisted curve $\mathscr{b} / S / \mathbb{F}_{p}$, if $N=p^{n}$ and $G_{K} \cong \mathbb{Z} /\left(p^{m}\right) \times \mathbb{Z} /\left(p^{l}\right)$ with $m \geq l \geq 1$, we set

$$
L_{K}:=\left\{H \leq G_{K} \mid H \text { and } G_{K} / H \text { are both cyclic }\right\} .
$$

For any $H \in L_{K}$ we say a $G_{K}$-structure $\phi: G_{K} \rightarrow \operatorname{Pic}_{\mathscr{C} / S}^{0}$ has component label $H$ if $H$ maps to the kernel of the $n$-fold relative Frobenius $F^{n}$ on the group scheme $\operatorname{Pic}_{\mathscr{C} / S}^{0}$ over $S$, and the resulting group scheme homomorphism $G_{K} / H \rightarrow$ $\operatorname{Pic}_{\mathscr{C} / S}^{0}\left[p^{n}\right] / \operatorname{ker}\left(F^{n}\right)$ is a $G_{K} / H$-structure in the sense of [Katz and Mazur 1985, $\S 1.5]$.

If $G_{K} \cong \mathbb{Z} /\left(p^{m}\right)$ (that is, $\left.l=0\right)$, then $X_{K}^{\mathrm{tw}} \cong X_{1}^{\mathrm{tw}}\left(p^{m}\right)$, and for $H \cong \mathbb{Z} /\left(p^{a}\right) \in$ $L_{K}$ we define $X_{K}^{\mathrm{tw}, H} \subset X_{K}^{\mathrm{tw}}$ to be the substack $X_{1}^{\mathrm{tw}}\left(p^{m}\right)^{(a, m-a)} \subset X_{1}^{\mathrm{tw}}\left(p^{m}\right)$ as in Definition 4.13. We still say that $X_{K}^{\mathrm{tw}, H}$ classifies $G_{K}$-structures of component label $H$.

So, for example, if $G_{K} \cong \mathbb{Z} /(d)$ for some $d \mid N, X_{K}^{\mathrm{tw}}$ is isomorphic to the stack $X_{1}^{\mathrm{tw}}(d)$, and if $G_{K} \cong(\mathbb{Z} /(d))^{2}$ then $X_{K}^{\mathrm{tw}}$ is isomorphic to the stack $X^{\mathrm{tw}}(d)$. For every such $K$, we have a closed immersion

$$
\iota^{K}: X_{K}^{\mathrm{tw}} \hookrightarrow \overline{\mathscr{K}}_{1,1}^{\prime}\left(\mathscr{B} \mu_{N}^{2}\right),
$$

given by precomposing a $G_{K}$-structure $\phi: G_{K} \rightarrow \operatorname{Pic}_{\mathscr{C} / T}^{0}$ with the canonical projection $(\mathbb{Z} /(N))^{2} \rightarrow G_{K}$. Together, these give a proper surjection

$$
\bigsqcup_{K \leq(\mathbb{Z} /(N))^{2}} x_{K}^{\mathrm{tw}} \rightarrow \overline{\mathscr{K}}_{1,1}^{\prime}\left(\mathscr{B} \mu_{N}^{2}\right)
$$

which is an isomorphism over $S[1 / N]$.

Let $k$ be a perfect field of characteristic $p$. The same argument that proves Lemma 2.18 immediately gives us:

Corollary 5.6. If $K \leq\left(\mathbb{Z} /\left(p^{n}\right)\right)^{2}$ such that $G_{K}:=\left(\mathbb{Z} /\left(p^{n}\right)\right)^{2} / K \cong \mathbb{Z} /\left(p^{m}\right) \times \mathbb{Z} /\left(p^{l}\right)$ with $l \leq m \leq n$, then $X_{K, k}^{\mathrm{tw}}$ is the disjoint union, with crossings at the supersingular points, of closed substacks $X_{K, k}^{\mathrm{tw}, H}$ for $H \in L_{K}$. $X_{K, k}^{\mathrm{tw}, H}$ classifies $G_{K}$-structures with component label $\mathrm{H}$.

As before, we let $\Lambda$ denote the set $\left\{(K, H) \mid K \leq\left(\mathbb{Z} /\left(p^{n}\right)\right)^{2}, H \in L_{K}\right\}$, modulo the equivalence relation generated by declaring $(K, H) \sim\left(K^{\prime}, \pi^{-1}(H)\right)$ whenever 
$K^{\prime} \leq K$ with corresponding quotient map $\pi: G_{K^{\prime}} \rightarrow G_{K}$ such that $\pi^{-1}(H) \in L_{K^{\prime}}$. We conclude:

Corollary 5.7. Let $k$ be a perfect field of characteristic $p . \overline{\mathscr{K}}_{1,1}^{\prime}\left(\mathscr{B}_{\left.\mu_{p^{n}}\right)_{k}}^{2}\right.$ is the disjoint union, with crossings at the supersingular points, of components $\overline{\mathscr{K}}_{1,1}^{\prime}\left(\Re_{B} \mu_{p^{n}}^{2}\right)_{k}^{\lambda}$ for $\lambda \in \Lambda$, where

$$
\overline{\mathscr{K}}_{1,1}^{\prime}\left(\mathscr{\Re} \mu_{p^{n}}^{2}\right)_{k}^{\lambda}:=\bigcup_{[(K, H)]=\lambda} X_{K, k}^{\mathrm{tw}, H},
$$

identifying each $X_{K, k}^{\mathrm{tw}, H}$ with a closed substack of $\overline{\mathscr{K}}_{1,1}^{\prime}\left(\mathscr{B} \mu_{p^{n}}^{2}\right)_{k}^{\lambda}$ via $\iota^{K}$. Each $X_{K, k}^{\mathrm{tw}, H}$ is "set-theoretically identified with $\overline{\mathscr{K}}_{1,1}^{\prime}\left(\mathscr{\Re} \mu_{p^{n}}^{2}\right)_{k}^{\lambda}$ " in the sense that

$$
\overline{\mathscr{K}}_{1,1}^{\prime}\left(\mathscr{B} \mu_{p^{n}}^{2}\right)_{k, \text { red }}^{\lambda}=X_{K, k, \text { red }}^{\mathrm{tw}, H}
$$

as substacks of $\overline{\mathscr{K}}_{1,1}^{\prime}\left(\mathscr{B} \mu_{p^{n}}\right)_{k \text {,red }}$ for all $(K, H)$ with $[(K, H)]=\lambda \in \Lambda$.

If $N=p^{n} N^{\prime}$ with $\left(p, N^{\prime}\right)=1$, for any $A \leq(\mathbb{Z} /(N))^{2}$ of order prime to $p$, let

$$
\overline{\mathscr{K}}_{1,1}^{\prime}\left(\mathscr{\Re} \mu_{N}^{2}\right)_{k}^{A} \subset \overline{\mathscr{K}}_{1,1}^{\prime}\left(\mathscr{B} \mu_{N}^{2}\right)_{k}
$$

be the union of the substacks $X_{K, k}^{\mathrm{tw}}$ for $A \leq K \leq(\mathbb{Z} /(N))^{2}$ with $(K: A)$ a power of $p$. Then similarly $\overline{\mathscr{K}}_{1,1}^{\prime}\left(\mathscr{B} \mu_{N}^{2}\right)_{k}^{A}$ is the disjoint union, with crossings at the supersingular points, of components $\overline{\mathscr{K}}_{1,1}^{\prime}\left(\mathscr{B} \mu_{N}^{2}\right)_{k}^{A, \lambda}$ for $\lambda \in \Lambda$, and $\overline{\mathscr{K}}_{1,1}^{\prime}\left(\mathscr{\Re} \mu_{N}^{2}\right)_{k}$ is the disjoint union of the open and closed substacks $\overline{\mathscr{K}}_{1,1}^{\prime}\left(\mathscr{B} \mu_{N}^{2}\right)_{k}^{A}$ for $A \leq(\mathbb{Z} /(N))^{2}$ of order prime to $p$.

The picture in the case $N=p$ is essentially the same as the picture for $\overline{\mathscr{K}}_{1,1}^{\circ}\left(\mathscr{B} \mu_{p}^{2}\right)_{k}$ $\simeq \mathcal{H}(p)_{k}$ (as discussed after Proposition 5.1), except now each component is proper:

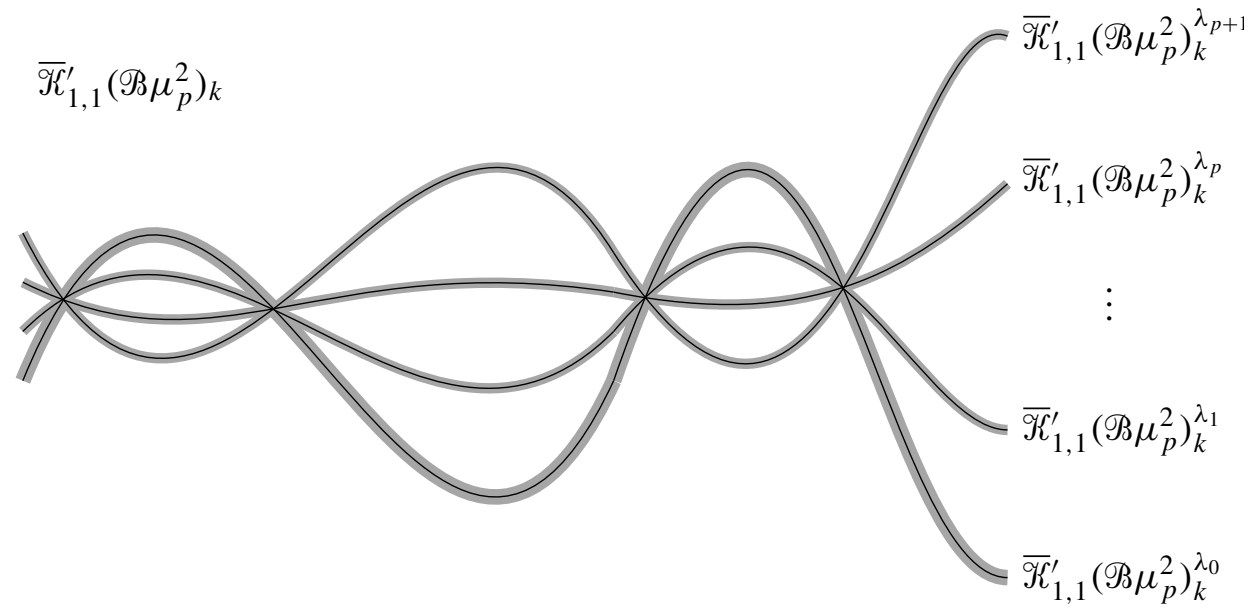




\section{Comparison with the classical moduli stacks}

As promised, we verify that the moduli stacks $X_{1}^{\mathrm{tw}}(N)$ and $X^{\mathrm{tw}}(N)$ are isomorphic to the corresponding classical moduli stacks, justifying the claim in [Abramovich et al. 2011] that we have recovered the Katz-Mazur regular models:

Theorem 6.1 (Restatement of Theorem 1.2). Over the base $S=\operatorname{Spec}(\mathbb{Z})$, there is a canonical isomorphism of algebraic stacks $X_{1}^{\mathrm{tw}}(N) \cong X_{1}(N)$ extending the identity map on $y_{1}(N)$, and a canonical isomorphism of algebraic stacks $X^{\mathrm{tw}}(N) \cong X(N)$ extending the identity map on $y(N)$.

We prove this after some preliminary results; the main point is to demonstrate that $X_{1}^{\mathrm{tw}}(N)$ and $X^{\mathrm{tw}}(N)$ are normal.

Proposition 6.2. The morphism $\pi: X_{1}^{\mathrm{tw}}(N) \rightarrow \bar{M}_{1,1}$ sending $(\mathscr{C}, \phi)$ to the coarse space $C$ of $\mathscr{C}$ is a representable morphism of stacks. In particular, $\chi_{1}^{\mathrm{tw}}(N)$ is Deligne-Mumford.

Similarly the natural morphism $X^{\mathrm{tw}}(N) \rightarrow \overline{\mathcal{M}}_{1,1}$ is representable, hence $X^{\mathrm{tw}}(N)$ is Deligne-Mumford.

Proof. We have already seen that $X_{1}^{\mathrm{tw}}(N)$ is an algebraic stack, so it suffices to show that for any object $(\mathscr{C}, \phi) \in X_{1}^{\mathrm{tw}}(N)(k)$ with $k$ an algebraically closed field, the natural map $\operatorname{Aut}(\mathscr{C}, \phi) \rightarrow \operatorname{Aut}(C)$ is a monomorphism of group schemes. Here $C / k$ is the coarse space of $\mathscr{b}$, and automorphisms are required to preserve the marking. It is obvious that $\operatorname{Aut}(\mathscr{C}, \phi) \rightarrow \operatorname{Aut}(C)$ is a monomorphism if $\mathscr{C}=C$ is a smooth elliptic curve over $k$, so by Lemma 3.17 we reduce to the case where $\mathscr{C} / k$ is a standard $\mu_{d}$-stacky Néron 1-gon for some $d \mid N$.

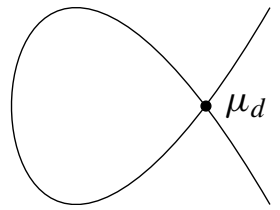

In this case an automorphism of $\mathscr{C}$ is an automorphism of the coarse space $C$, together with an automorphism of the $\mu_{d}$-gerbe in $\mathscr{C}$ lying over the node of $C$. Thus

$$
\operatorname{Aut}(\mathscr{C}) \cong \operatorname{Aut}(C) \times \operatorname{Aut}\left(\mathscr{B} \mu_{d, k}\right) .
$$

The only nontrivial automorphism of $C$ preserving the marked point $1 \in C$ is the automorphism $\iota: C \rightarrow C$ induced by the inversion automorphism of $\mathbb{G}_{m}$. We have $\operatorname{Aut}\left(\mathscr{B} \mu_{d}\right) \cong \mu_{d}$, and the automorphism of $\operatorname{Pic}_{\mathscr{C} / k}^{0} \cong \mathbb{G}_{m} \times \mathbb{Z} /(d)$ induced by $(0, \zeta) \in \operatorname{Aut}(\mathscr{C}) \cong\langle\iota\rangle \times \mu_{d}$ sends $(\eta, a)$ to $\left(\zeta^{a} \eta, a\right)$. Since $\phi: \mathbb{Z} /(N) \rightarrow \operatorname{Pic}_{\mathscr{C} / k}^{0}$ meets every component, the only automorphisms of $\mathscr{b}$ than can possibly preserve $\phi$ are the automorphisms $\langle\iota\rangle \times\{0\} \subset \operatorname{Aut}(\mathscr{C})$ (see [Conrad 2007, proof of 3.1.8]). Thus $\operatorname{Aut}(\mathscr{C}, \phi) \subset\langle\iota\rangle \times\{0\} \cong \operatorname{Aut}(C)$. 
The same argument applies to $X^{\mathrm{tw}}(N)$.

Corollary 6.3. $X_{1}^{\mathrm{tw}}(N)$ and $X^{\mathrm{tw}}(N)$ are finite over $\bar{M}_{1,1}$.

Proof. By Theorems 4.6 and 5.3, the natural maps $X_{1}^{\mathrm{tw}}(N) \rightarrow \bar{M}_{1,1}$ and $X^{\mathrm{tw}}(N) \rightarrow$ $\bar{M}_{1,1}$ are proper and quasifinite; since they are also representable, they are finite.

Let $\infty \hookrightarrow \bar{M}_{1,1}$ denote the closed substack classifying 1-marked genus-1 stable curves whose geometric fibers are singular. Let $X_{1}^{\mathrm{tw}}(N)^{\infty}=X_{1}^{\mathrm{tw}}(N) \times{\overline{\bar{M}_{1,1}}} \infty$ and $X^{\mathrm{tw}}(N)^{\infty}=X^{\mathrm{tw}}(N) \times \bar{M}_{1,1} \infty$. Exactly analogously to [Conrad 2007, 2.1.12], formation of these closed substacks is compatible with arbitrary base change.

Proposition 6.4. The proper flat morphisms $X_{1}^{\mathrm{tw}}(N) \rightarrow \operatorname{Spec}(\mathbb{Z})$ and $X^{\mathrm{tw}}(N) \rightarrow$ $\operatorname{Spec}(\mathbb{Z})$ are Cohen-Macaulay (of pure relative dimension 1 ).

Proof. Let $X$ denote $X_{1}^{\mathrm{tw}}(N)$ or $\mathcal{X}^{\mathrm{tw}}(N)$. The canonical morphism $X \rightarrow \bar{M}_{1,1}$ is finite (by Corollary 6.3) and flat (by Theorems 4.6 and 5.3), and the structural morphism $\bar{M}_{1,1} \rightarrow \operatorname{Spec}(\mathbb{Z})$ is Cohen-Macaulay (see [Conrad 2007, 3.3.1]), so by [Bourbaki 1998, 2.7.9, Corollary 3], the composite $X \rightarrow \operatorname{Spec}(\mathbb{Z})$ is CohenMacaulay.

Lemma 6.5. $X_{1}^{\mathrm{tw}}(N)^{\infty}$ and $X^{\mathrm{tw}}(N)^{\infty}$ are relative effective Cartier divisors over $\operatorname{Spec}(\mathbb{Z})$ in $X_{1}^{\mathrm{tw}}(N)$ and $X^{\mathrm{tw}}(N)$, respectively.

Proof. Here we are using the notion of a Cartier divisor on a Deligne-Mumford stack; see [Arbarello et al. 2011, Chapter XIII]. For $X=X_{1}^{\mathrm{tw}}(N)$ or $X=X^{\mathrm{tw}}(N)$, the closed substack $X^{\infty}$ is the pullback $X \times \bar{M}_{1,1} \infty$. We know $\infty \subset \bar{M}_{1,1}$ is a relative effective Cartier divisor over $\operatorname{Spec}(\mathbb{Z})$ (meaning an effective Cartier divisor which is flat over $\operatorname{Spec}(\mathbb{Z})$ ), and by Theorems 4.6 and 5.3 the morphism $X \rightarrow \bar{M}_{1,1}$ is flat. Cartier divisors are preserved by flat morphisms (see [Fulton 1998, §1.7]), so $X^{\infty}$ is an effective Cartier divisor in $X$. Since $X \rightarrow \bar{M}_{1,1}$ is flat, so is $X^{\infty} \rightarrow \infty$, so $X^{\infty}$ is flat over $\operatorname{Spec}(\mathbb{Z})$, that is, $X^{\infty}$ is a relative effective Cartier divisor in $X$ over $\operatorname{Spec}(\mathbb{Z})$.

Corollary 6.6. $X_{1}^{\mathrm{tw}}(N)$ and $X^{\mathrm{tw}}(N)$ are normal.

Proof. This is proven in an identical manner to [Conrad 2007, 4.1.4]. The stacks $X_{1}^{\mathrm{tw}}(N)$ and $X^{\mathrm{tw}}(N)$ are Deligne-Mumford, and from [Abramovich et al. 2003, 3.0.2] we know $X_{1}^{\mathrm{tw}}(N) \otimes_{\mathbb{Z}} \mathbb{Z}[1 / N]$ and $X^{\mathrm{tw}}(N) \otimes_{\mathbb{Z}} \mathbb{Z}[1 / N]$ are smooth over $\operatorname{Spec}(\mathbb{Z}[1 / N])$. In particular, they are regular at any characteristic-0 points. Furthermore, by Proposition 6.4 they are Cohen-Macaulay over $\operatorname{Spec}(\mathbb{Z})$ of pure relative dimension 1. As in [Conrad 2007, 4.1.4], we can conclude from Serre's criterion for normality that it suffices to prove that these stacks are regular away from some relative effective Cartier divisor, since such a divisor cannot contain any codimension-1 points of positive residue characteristic. Use the divisors $X_{1}^{\mathrm{tw}}(N)^{\infty}$ and $X^{\mathrm{tw}}(N)^{\infty}$; their complements are $y_{1}(N)$ and $y(N)$, which are regular by [Katz and Mazur 1985, 5.1.1]. 
Proof of Theorem 6.1. $X_{1}^{\mathrm{tw}}(N)$ and $X^{\mathrm{tw}}(N)$ are finite, flat, and normal over $\bar{M}_{1,1}$, so they are naturally identified with the normalizations (in the sense of [Deligne and Rapoport 1973, IV.3.3]) of $\bar{M}_{1,1}$ in $\left.X_{1}^{\mathrm{tw}}(N)\right|_{\mathcal{M}_{1,1}}=y_{1}(N)$ and $\left.X^{\mathrm{tw}}(N)\right|_{\mathcal{M}_{1,1}}=y(N)$, respectively; see [Conrad 2007, 4.1.5].

We now give a moduli interpretation of the equivalence $X_{1}(N) \simeq X_{1}^{\mathrm{tw}}(N)$. Let $S$ be a scheme, $E / S$ be a generalized elliptic curve, and $P \in E^{\operatorname{sm}}(S)[N]$ be a $\left[\Gamma_{1}(N)\right]$-structure on $E$. From this data we want to construct a pair $\left(\mathscr{C}_{P}, \phi_{P}\right)$, where $\mathscr{C}_{P} / S$ is a 1-marked genus-1 twisted stable curve with nonstacky marking, and $\phi_{P}: \mathbb{Z} /(N) \rightarrow \operatorname{Pic}_{\mathscr{C}_{P} / S}^{0}$ is a $\left[\Gamma_{1}(N)\right]$-structure on $\mathscr{C}_{P}$.

If $E / S$ is a smooth elliptic curve, there is nothing to show: we simply take $\left(\mathscr{C}_{P}, \phi_{P}\right)=\left(E, \phi_{P}\right)$ where $\phi_{P}: \mathbb{Z} /(N) \rightarrow \operatorname{Pic}_{E / S}^{0} \cong E$ sends $1 \mapsto P$. Therefore to construct $\left(\mathscr{C}_{P}, \phi_{P}\right)$ in general, we may restrict to the open subscheme of $S$ where $E / S$ has no supersingular geometric fibers; once we have constructed $\left(\mathscr{C}_{P}, \phi_{P}\right)$ in this case, we only need to check that it agrees with our previous construction for ordinary elliptic curves.

For the rest of the construction, assume that $E / S$ has no supersingular geometric fibers.

Note that by [Conrad 2007, 4.2.3], fppf locally on $S$ there exists a generalized elliptic curve $E^{\prime} / S$, whose singular geometric fibers are $N$-gons, together with an open $S$-immersion $\iota: E^{\mathrm{sm}} \hookrightarrow E^{\prime \text { sm }}$ of group schemes over $S$. In particular, by [Deligne and Rapoport 1973, II.1.20] the group scheme $E^{\prime \mathrm{sm}}[N] / S$ is finite and flat of constant rank $N^{2}$.

Since $E^{\prime} / S$ has no supersingular geometric fibers and all its singular geometric fibers are $N$-gons, it follows that fppf locally on $S$, there exists a $[\Gamma(N)]$-structure $(Q, R)$ on $E^{\prime}$ such that:

- the relative effective Cartier divisor

$$
D:=\sum_{a \in \mathbb{Z} /(N)}[a \cdot Q]
$$

in $E^{\prime \text { sm }}$ is étale over $S$ and

- $R$ meets the identity component of every geometric fiber of $E^{\prime} / S$.

The choice of $Q$ and the pairing

$$
e_{N}: E^{\prime s \mathrm{~s}}[N] \times E^{\prime \mathrm{sm}}[N] \rightarrow \mu_{N}
$$

induce a canonical isomorphism $E^{\prime \mathrm{sm}}[N] / D \cong E^{\prime \mathrm{sm}}[N] / D \times\{Q\} \cong \mu_{N}$. Identifying $E^{\prime s m}[N] / D$ with its image in the $N$-torsion of the generalized elliptic curve $C:=$ $E^{\prime} / D$ (a generalized elliptic curve whose singular fibers are 1-gons), the group law of $C$ and the above isomorphism give us an action of $\mu_{N}$ on $C$, making $C$ a 
$\mu_{N}$-torsor over the twisted curve $\mathscr{C}:=\left[C / \mu_{N}\right]=\left[E^{\prime} / E^{\prime \mathrm{sm}}[N]\right]=\left[E / E^{\mathrm{sm}}[N]\right]$. Write

$$
C=\underline{\operatorname{Spec}} \mathscr{C}\left(\bigoplus_{a \in \mathbb{Z} /(N)} \mathscr{G}_{a}\right) \stackrel{\pi}{\longrightarrow} \mathscr{C},
$$

where each $\mathscr{G}_{a}$ is an invertible $\mathscr{O}_{\mathscr{C}}$-module, with the grading and algebra structure corresponding to the structure of $C$ as a $\mu_{N}$-torsor over $\mathscr{b}$.

The image $\bar{R}$ of $R$ in $C$ is a $\left[\Gamma_{1}(N)\right]$-structure on $C$, so we get a $\mu_{N}$-torsor

$$
T:=\underline{\operatorname{Spec}_{C}}\left(\bigoplus_{b \in \mathbb{Z} /(N)} \mathscr{L}\left((b \cdot \bar{R})-\left(0_{C}\right)\right)\right)
$$

over $C$; the $\mu_{N}$-action on $T$ corresponds to the $\mathbb{Z} /(N)$-grading and the algebra structure on

$$
\bigoplus_{b \in \mathbb{Z} /(N)} \mathscr{L}\left((b \cdot \bar{R})-\left(0_{C}\right)\right)
$$

comes from the group law on $C^{\mathrm{sm}}$ and the canonical isomorphism $C^{\mathrm{sm}} \cong \mathrm{Pic}_{C / S}^{0}$.

Since $C$ is a $\mu_{N}$-torsor over $\mathscr{C}$, if $\mathscr{L} \in \operatorname{Pic}(C)$ we have a canonical decomposition

$$
\pi_{*} \mathscr{L}=\bigoplus_{a \in \mathbb{Z} /(N)} \mathscr{L}_{a}
$$

where each $\mathscr{L}_{a}$ is an invertible sheaf on $\mathscr{C}$ and $\zeta \in \mu_{N}$ acts on $\mathscr{L}_{a}$ via multiplication by $\zeta^{a}$. In particular this applies to the invertible sheaf $\mathscr{L}=\mathscr{L}\left((b \cdot \bar{R})-\left(0_{C}\right)\right)$, giving us a canonical decomposition

$$
\pi_{*} \mathscr{L}\left((b \cdot \bar{R})-\left(0_{C}\right)\right)=\bigoplus_{a \in \mathbb{Z} /(N)} \mathscr{L}_{(a, b)} .
$$

We have $\mathscr{L}_{0,0}=\mathscr{G}_{0}=\mathcal{O}_{\mathscr{C}}$, and the isomorphisms

$$
\mathscr{L}\left(\left(b_{0} \cdot \bar{R}\right)-\left(0_{C}\right)\right) \otimes_{0_{C}} \mathscr{L}\left(\left(b_{1} \cdot \bar{R}\right)-\left(0_{C}\right)\right) \cong \mathscr{L}\left(\left(\left(b_{0}+b_{1}\right) \cdot \bar{R}\right)-\left(0_{C}\right)\right)
$$

(coming from the algebra structure of $\bigoplus_{b} \mathscr{L}\left((b \cdot \bar{R})-\left(0_{C}\right)\right)$ ) induce isomorphisms

$$
\mathscr{L}_{\left(a_{0}, b_{0}\right)} \otimes_{\mathcal{O}_{\varphi}} \mathscr{L}_{\left(a_{1}, b_{1}\right)} \cong \mathscr{L}_{\left(a_{0}+a_{1}, b_{0}+b_{1}\right)}
$$

for all $\left(a_{0}, b_{0}\right),\left(a_{1}, b_{1}\right) \in(\mathbb{Z} /(N))^{2}$, giving us a canonical algebra structure on the direct sum

$$
\bigoplus_{(a, b) \in(\mathbb{Z} /(N))^{2}} \mathscr{L}_{(a, b)} .
$$

Identifying our original $\left[\Gamma_{1}(N)\right]$-structure $P$ with its image in $E^{\prime s m}(S)[N]$, there exists some $\left(a_{0}, b_{0}\right) \in(\mathbb{Z} /(N))^{2}$ with $P=a_{0} \cdot Q+b_{0} \cdot R \in E^{\prime \mathrm{sm}}[N]$. This determines 
a $\mu_{N}$-torsor

$$
\mathscr{T}:=\underline{\operatorname{Spec}}_{\mathscr{C}}\left(\bigoplus_{c \in \mathbb{Z} /(N)} \mathscr{L}_{\left(c a_{0}, c b_{0}\right)}\right)
$$

over $\mathscr{C}$, corresponding to a morphism $\mathscr{C} \rightarrow \mathscr{B} \mu_{N}$. Here $\bigoplus \mathscr{L}_{\left(c a_{0}, c b_{0}\right)}$ is viewed as a sub- $\Theta_{\mathscr{C}}$-algebra of the algebra $\bigoplus \mathscr{L}_{(a, b)}$.

Definition 6.7. We define $\mathscr{C}_{P} \rightarrow \mathscr{B} \mu_{N}$ to be the relative coarse moduli space of the above morphism $\mathscr{C} \rightarrow \mathscr{B} \mu_{N}$, and we write $\phi_{P}: \mathbb{Z} /(N) \rightarrow \mathrm{Pic}_{\mathscr{C}_{P} / S}^{0}$ for the corresponding group scheme homomorphism.

It is immediate that $\phi_{P}$ is a $\left[\Gamma_{1}(N)\right]$-structure on the twisted curve $\mathscr{C}_{P}$.

Lemma 6.8. ( $\left.\mathscr{C}_{P}, \phi_{P}\right)$ is independent of the choice of $\left(a_{0}, b_{0}\right)$ with $P=a_{0} \cdot Q+b_{0}$. $R$, and of the choice of generalized elliptic curve $E^{\prime}$ and $[\Gamma(N)]$-structure $(Q, R)$ on $E^{\prime}$ such that $D=\sum[a \cdot Q]$ is étale over $S$ and $R$ meets the fiberwise identity components of $E^{\prime} / S$.

Proof. First of all, if $E / S$ is an ordinary elliptic curve, then $E^{\prime}=E$. Our construction defines a map $E[N] \rightarrow \operatorname{Pic}_{E / S}^{0}[N] \cong E[N]$, which in fact is simply the identity map (which in particular is independent of the choice of $\left(a_{0}, b_{0}\right)$ and the $[\Gamma(N)]$-structure $(Q, R))$. To see this, recall that by Lemma 3.22 we may identify the generalized elliptic curve $C=E /\langle Q\rangle$ (viewed as a $\mu_{N}$-torsor over $E \cong[E / E[N]]$ as discussed above) with the $\mu_{N}$-torsor

$$
\operatorname{Spec}_{E}\left(\bigoplus_{a \in \mathbb{Z} /(N)} \mathscr{L}\left((a \cdot Q)-\left(0_{E}\right)\right)\right)
$$

over $E$. So in the notation of the above construction, $\mathscr{C}=E$ and $\mathscr{G}_{a}=\mathscr{L}_{(a, 0)}=$ $\mathscr{L}\left((a \cdot Q)-\left(0_{E}\right)\right)$. We have

$$
\begin{aligned}
\pi_{*} \mathscr{L}\left((\bar{R})-\left(0_{C}\right)\right) & \cong \bigoplus_{a \in \mathbb{Z} /(N)}\left(\mathscr{G}_{a} \otimes \mathscr{L}\left((R)-\left(0_{E}\right)\right)\right) \\
& \cong \bigoplus_{a \in \mathbb{Z} /(N)} \mathscr{L}\left((a \cdot Q+R)-\left(0_{E}\right)\right) .
\end{aligned}
$$

So the map defined in the above construction sends $Q$ to $\mathscr{L}_{(1,0)}=\mathscr{L}\left((Q)-\left(0_{E}\right)\right)$ and $R$ to $\mathscr{L}_{(0,1)}=\mathscr{L}\left((R)-\left(0_{E}\right)\right)$. Composing with the usual isomorphism $\mathrm{Pic}_{E / S}^{0} \cong E$ yields the identity map on $E[N]$.

To complete the proof in the case where $E / S$ is not necessarily smooth, it suffices to consider the case where $S$ is the spectrum of an algebraically closed field $k$ and $E / k$ is a Néron $d$-gon (for some $d \mid N$ ). $E^{\prime} / k$ is then a Néron $N$-gon, and our Drinfeld basis $(Q, R)$ was chosen so that $\langle Q\rangle$ meets every irreducible component of $E^{\prime}$ and $\langle R\rangle$ lies on the identity component. We may therefore choose 
an isomorphism $E^{\prime \mathrm{sm}} \cong \mathbb{Z} /(N) \times \mu_{N}$ such that $Q=(1,1)$ and $R=(0, \zeta)$ for some $\zeta \in \mu_{N}^{\times}(k)$.

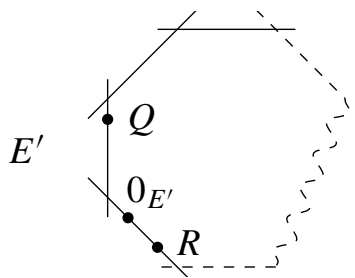

Then if $P=a_{0} \cdot Q+b_{0} \cdot R=a_{1} \cdot Q+b_{1} \cdot R$, it follows that $a_{0}=a_{1}$ and $\zeta^{b_{0}}=\zeta^{b_{1}}$, the latter of which implies that $b_{0} \cdot \bar{R}=b_{1} \cdot \bar{R}$. Thus $\mathscr{L}\left(\left(b_{0} \cdot \bar{R}\right)-\left(0_{C}\right)\right)=\mathscr{L}\left(\left(b_{1} \cdot \bar{R}\right)-\left(0_{C}\right)\right)$, so $\mathscr{L}_{\left(a_{0}, b_{0}\right)}=\mathscr{L}_{\left(a_{1}, b_{1}\right)}$, hence $\left(\mathscr{C}_{P}, \phi_{P}\right)$ is independent of the choice of $\left(a_{0}, b_{0}\right)$.

Now we must check that $\left(\mathscr{C}_{P}, \phi_{P}\right)$ is independent of the choice of $\left(E^{\prime},(Q, R)\right)$. $E^{\prime} / k$ is a Néron $N$-gon, so the choice of $E^{\prime}$ is unique up to composition with an automorphism of $E^{\prime}$ fixing $E^{\mathrm{sm}} \subset E^{\prime \mathrm{sm}}$. $E^{\prime}$ is the special fiber of an $N$-gon Tate curve $\mathscr{E}^{\prime} / k \llbracket q^{1 / N} \rrbracket$. Let $\mathscr{C}=\left[\mathscr{E}^{\prime} / \mathscr{C}^{\prime} \mathrm{sm}[N]\right]$, so $\mathscr{C}_{k}=\left[E^{\prime} / E^{\prime \mathrm{sm}}[N]\right]=\left[E / E^{\mathrm{sm}}[N]\right]$.

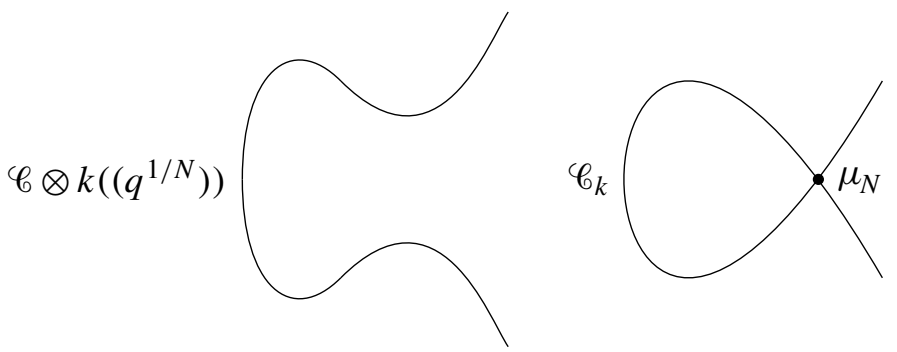

We may choose an isomorphism $\mathscr{E}^{\prime} \mathrm{sm}[N] \cong \mathbb{Z} /(N) \times \mu_{N}$ of finite flat group schemes over $k \llbracket q^{1 / N} \rrbracket .(Q, R)$ extends to a $[\Gamma(N)]$-structure $(2, \mathscr{R})$ on $\mathscr{E}^{\prime}$ with $\mathscr{D}=\sum(a \cdot \mathscr{2})$ étale over $k \llbracket q^{1 / N} \rrbracket$ (and of course $\mathscr{R}$ meets the identity component of every geometric fiber of $\left.\mathscr{E}^{\prime} / k \llbracket q^{1 / N} \rrbracket\right)$. Given such a $[\Gamma(N)]$-structure on $\mathscr{E}^{\prime}$, our construction defines a group scheme homomorphism

$$
\mathscr{C}^{\prime \prime s m}[N] \rightarrow \operatorname{Pic}_{\mathscr{C} / k \llbracket q^{1 / N} \rrbracket}^{0}[N] .
$$

Both of these are finite flat group schemes over $k \llbracket q^{1 / N} \rrbracket$ which are isomorphic to $\mathbb{Z} /(N) \times \mu_{N}$, and $\underline{\operatorname{End}}\left(\mathbb{Z} /(N) \times \mu_{N}\right)$ is finite (hence proper) over $k \llbracket q^{1 / N} \rrbracket$. Since

$$
\mathscr{E}^{\prime \mathrm{sm}}[N] \otimes k\left(\left(q^{1 / N}\right)\right) \rightarrow \mathrm{Pic}_{\mathscr{C} / k \llbracket q^{1 / N} \rrbracket}^{0}[N] \otimes k\left(\left(q^{1 / N}\right)\right)
$$

is independent of the choice of $[\Gamma(N)]$-structure over $k\left(\left(q^{1 / N}\right)\right)\left(\right.$ as $\mathscr{E}^{\prime} \otimes k\left(\left(q^{1 / N}\right)\right)$ is an elliptic curve), we conclude that $\mathscr{C}^{\prime \mathrm{sm}}[N] \rightarrow \operatorname{Pic}_{\mathscr{C} / k \llbracket q^{1 / N} \rrbracket}^{0}[N]$ is independent of the choice of $(2, \mathscr{R})$. Thus in particular $E^{\prime \mathrm{sm}}[N] \rightarrow \operatorname{Pic}_{\mathscr{C}_{k} / k}^{0}[N]$ is independent of the choice of $(Q, R)$ and the resulting homomorphism $E^{\mathrm{sm}}[N] \rightarrow \mathrm{Pic}_{\mathscr{C}_{k} / k}^{0}[N]$ is independent of the choice of $E^{\prime}$. 
Thus by descent, $\left(\mathscr{C}_{P}, \phi_{P}\right) \in X_{1}^{\mathrm{tw}}(N)(S)$ is well defined globally over our initial base scheme $S$ (even allowing supersingular fibers) and depends only on the pair $(E, P) \in X_{1}(N)(S)$. We define our map $X_{1}(N) \rightarrow X_{1}^{\mathrm{tw}}(N)$ by sending $(E, P)$ to $\left(\mathscr{C}_{P}, \phi_{P}\right)$.

Corollary 6.9. Over any base scheme $S$, the morphism $X_{1}(N) \rightarrow X_{1}^{\mathrm{tw}}(N)$ sending $(E, P)$ to $\left(\mathscr{C}_{P}, \phi_{P}\right)$ is an isomorphism of algebraic stacks.

Similarly, given a generalized elliptic curve $E / S$ which is equipped with a $[\Gamma(N)]$-structure $\left(P_{1}, P_{2}\right)$, the above procedure produces a $[\Gamma(N)]$-structure $\phi_{\left(P_{1}, P_{2}\right)}$ on the twisted curve $\mathscr{C}_{E}:=\left[E / E^{\mathrm{sm}}[N]\right]$.

Corollary 6.10. Over any base scheme $S$, the morphism $X(N) \rightarrow X^{\mathrm{tw}}(N)$ sending $\left(E,\left(P_{1}, P_{2}\right)\right)$ to $\left(\mathscr{C}_{E}, \phi_{\left(P_{1}, P_{2}\right)}\right)$ is an isomorphism of algebraic stacks.

\section{Other compactified moduli stacks of elliptic curves}

It is worth noting that the techniques in the proof of Theorem 4.6 are easily adapted to prove properness of the natural analogues in our current setting of well-known modular compactifications of other various moduli stacks of elliptic curves with extra structure, even when these moduli stacks do not naturally lie in a moduli stack of twisted stable maps:

Definition 7.1. Let $\mathscr{C} / S$ be a 1 -marked genus-1 twisted stable curve with nonstacky marking.

(i) A $\left[\Gamma_{0}(N)\right]$-structure on $\mathscr{C}$ is a finite locally free $S$-subgroup scheme $G$ of $\operatorname{Pic}_{\mathscr{C} / S}^{0}$ of rank $N$ over $S$ which is cyclic (fppf locally admits a $\mathbb{Z} /(N)$-generator), such that for every geometric point $\bar{p} \rightarrow S, G_{\bar{p}}$ meets every irreducible component of $\operatorname{Pic}_{\mathscr{C}_{\bar{p}} / k(\bar{p})}^{0}$. We write $X_{0}^{\mathrm{tw}}(N)$ for the stack over $S$ associating to $T / S$ the groupoid of pairs $(\mathscr{C}, G)$, where $\mathscr{C} / T$ is a 1 -marked genus- 1 twisted stable curve with nonstacky marking, and $G$ is a $\left[\Gamma_{0}(N)\right]$-structure on $\mathscr{b}$.

(ii) A balanced $\left[\Gamma_{1}(N)\right]$-structure (see [Katz and Mazur 1985, §3.3]) on $\mathscr{C}$ is an fppf short exact sequence of commutative group schemes over $S$

$$
0 \rightarrow K \rightarrow \mathrm{Pic}_{\mathscr{C} / S}^{0}[N] \rightarrow K^{\prime} \rightarrow 0,
$$

where $K$ and $K^{\prime}$ are locally free of rank $N$ over $S$, together with sections $P \in K(S)$ and $P^{\prime} \in K^{\prime}(S)$ which are $\mathbb{Z} /(N)$-generators of $K$ and $K^{\prime}$ in the sense of [Katz and Mazur 1985, §1.4]. We write $X_{1}^{\text {bal,tw }}(N)$ for the stack over $S$ associating to $T / S$ the groupoid of pairs $(\mathscr{b}, \dagger)$, where $\mathscr{C} / T$ is a 1-marked genus-1 twisted stable curve with nonstacky marking, and $\dagger$ is a balanced $\left[\Gamma_{1}(N)\right]$-structure on $\mathscr{C}$.

(iii) An [N-Isog]-structure (see [Katz and Mazur 1985, §6.5]) on $\mathscr{C}$ is a finite locally free commutative $S$-subgroup scheme $G \subset \operatorname{Pic}_{\mathscr{C} / S}^{0}[N]$ of rank $N$ over $S$, 
such that for every geometric point $\bar{p} \rightarrow S, G_{\bar{s}}$ meets every irreducible component of $\operatorname{Pic}_{\mathscr{C}_{\bar{p}} / k(\bar{p})}^{0}$. We write $X^{\mathrm{tw}}(N$-Isog) for the stack over $S$ associating to $T / S$ the groupoid of pairs $(\mathscr{C}, G)$, where $\mathscr{C} / T$ is a 1-marked genus-1 twisted stable curve with nonstacky marking, and $G$ is an [N-Isog]-structure on $\mathscr{C}$.

(iv) If $N$ and $n$ are positive integers such that $\operatorname{ord}_{p}(n) \leq \operatorname{ord}_{p}(N)$ for all primes $p$ dividing both $N$ and $n$, a [ $\left.\Gamma_{1}(N ; n)\right]$-structure (see [Conrad 2007, 2.4.3]) on $\mathscr{b}$ is a pair $(\phi, G)$, where

- $\phi: \mathbb{Z} /(N) \rightarrow \operatorname{Pic}_{\mathscr{C} / S}^{0}$ is a $\mathbb{Z} /(N)$-structure in the sense of [Katz and Mazur $1985, \S 1.5]$,

- $G \subset \mathrm{Pic}_{\mathscr{C} / S}^{0}$ is a finite locally free $S$-subgroup scheme which is cyclic of order $n$,

- the degree- $N n$ relative effective Cartier divisor

$$
\sum_{a=0}^{N-1}(\phi(a)+G)
$$

in $\mathrm{Pic}_{\mathscr{C} / S}^{0}$ meets every irreducible component of each geometric fiber of $\mathrm{Pic}_{\mathscr{C} / S}^{0}$ over $S$, and

- for all primes $p$ dividing both $N$ and $n$, for $e=\operatorname{ord}_{p}(n)$ we have an equality of closed subschemes of $\mathrm{Pic}_{\mathscr{C} / S}^{0}$

$$
\sum_{a=0}^{p^{e}-1}\left(\left(N / p^{e}\right) \cdot \phi(a)+G\left[p^{e}\right]\right)=\operatorname{Pic}_{\mathscr{C} / S}^{0}\left[p^{e}\right] .
$$

We write $X_{1}^{\mathrm{tw}}(N ; n)$ for the stack over $S$ associating to $T / S$ the groupoid of tuples $(\mathscr{C},(\phi, G))$, where $\mathscr{C} / T$ is a 1 -marked genus-1 twisted stable curve with nonstacky marking, and $(\phi, G)$ is a $\left[\Gamma_{1}(N ; n)\right]$-structure on $\mathscr{C}$.

Corollary 7.2. The stacks $X_{0}^{\mathrm{tw}}(N), X_{1}^{\mathrm{bal}, \mathrm{tw}}(N), X^{\mathrm{tw}}\left(N\right.$-Isog), and $X_{1}^{\mathrm{tw}}(N ; n)$ are algebraic stacks which are flat and locally finitely presented over $S$, with local complete intersection fibers. They are proper and quasifinite over $\bar{M}_{1,1}$, and each is isomorphic to the corresponding moduli stack for generalized elliptic curves.

As shown explicitly for the stacks $X_{1}^{\mathrm{tw}}(N)$ and $X^{\mathrm{tw}}(N)$ earlier in this paper, one may study the reductions modulo $p$ of these moduli stacks, and one finds that over a perfect field of characteristic $p$, each stack is a disjoint union with crossings at the supersingular points of various closed substacks, which come naturally equipped with moduli interpretations extending the definitions given in [Katz and Mazur 1985] (except for $X_{1}^{\mathrm{tw}}(N ; n)$, which is not studied there).

Question 7.3. If $\mathscr{C} / S$ is a 1 -marked genus- 1 twisted stable curve with nonstacky marking, the group scheme $\mathrm{Pic}_{\mathscr{C} / S}^{0}$ behaves just like the smooth part of a generalized 
elliptic curve. Over the Zariski open set of $S$ where $\mathscr{b} \rightarrow S$ is smooth, it agrees with $C$ (which is in this case a smooth elliptic curve); if $\bar{p} \rightarrow S$ is a geometric point such that $C_{\bar{p}}$ is singular, then $\operatorname{Pic}_{\mathscr{C}_{\bar{p}} / k(\bar{p})}^{0} \cong \mathbb{G}_{m} \times \mathbb{Z} /(N)$ for some $N$, and this is just the smooth part of a Néron $N$-gon over $k(\bar{p})$. Is there a natural way to exhibit $\mathrm{Pic}_{\mathscr{C} / S}^{0}$ as the smooth part of a generalized elliptic curve, giving an equivalence between the stack of generalized elliptic curves over $S$ and the stack of 1-marked genus-1 twisted stable curves over $S$ with nonstacky marking? More precisely, is there a natural way of defining compactified Jacobians of twisted curves, such that the degree-0 compactified Jacobian of a standard $\mu_{d}$-stacky Néron 1-gon is a Néron $d$-gon?

\section{Appendix: On moduli of curves of higher genus}

As in the case of elliptic curves, stacks of twisted stable maps allow for natural compactifications of stacks of genus- $g$ curves equipped with certain extra structure. For example,

$$
\overline{\mathscr{K}}_{g, 0}^{\circ}\left(\mathscr{\Re} \mu_{N}\right):=\overline{\mathscr{K}}_{g, 0}\left(\mathscr{\Re} \mu_{N}\right) \times \bar{M}_{g} \mu_{g}
$$

classifies pairs $(C / S, \phi)$, where $C / S$ is a smooth genus- $g$ curve and $\phi: \mathbb{Z} /(N) \rightarrow$ $\operatorname{Pic}_{C / S}^{0}$, which we view as an $N$-torsion point in $\operatorname{Pic}_{C / S}^{0}=\operatorname{Jac}(C / S)$. This stack is naturally contained in the proper algebraic stack $\overline{\mathscr{K}}_{g, 0}\left(\mathscr{B}_{\mu_{N}}\right)$ as an open dense substack. However, the situation becomes considerably more complicated when we attempt to use this to obtain proper moduli stacks of curves with level structure, for example, replacing " $N$-torsion points" with "points of exact order $N$ ".

Over $\mathbb{Z}[1 / N]$, we have a stack $\bar{M}_{g}^{(N)}$ of twisted curves with level- $N$ structure, studied in [Abramovich et al. 2003, §6]; this is a smooth proper modular compactification of the stack classifying (not necessarily symplectic) Jacobi level- $N$ structures on smooth genus- $g$ curves, as in [Deligne and Mumford 1969, 5.4]. One may be tempted to proceed as follows:

Definition A.1. Let $\mathscr{b} / S$ be an unmarked genus- $g(g>1)$ twisted stable curve over a scheme $S$. A full level- $N$ structure on $\mathscr{C}$ is a group scheme homomorphism $\phi:(\mathbb{Z} /(N))^{2 g} \rightarrow \operatorname{Pic}_{\mathscr{C} / S}^{0}$ such that $\left\{\phi(a) \mid a \in(\mathbb{Z} /(N))^{2}\right\}$ is a full set of sections for the finite flat group scheme $\operatorname{Pic}_{\mathscr{C} / S}^{0}[N]$ over $S$ in the sense of [Katz and Mazur $1985, \S 1.8]$.

We write $\overline{\mathcal{M}}_{g}^{(N), \text { tw }}$ for the substack of $\overline{\mathscr{K}}_{g, 0}\left(\mathscr{B}_{\mu}^{2 g}\right)$ associating to $T / S$ the groupoid of pairs $(\mathscr{C}, \phi)$, where $\mathscr{C} / T$ is an unmarked genus- $g$ twisted stable curve and $\phi$ is a full level- $N$ structure on $\mathscr{C}$.

Unfortunately, this is not the "right" definition. By this we mean that we would like the stack $\overline{\mathcal{M}}_{g}^{(N), \text { tw }}$ to be a closed substack of $\overline{\mathscr{K}}_{g, 0}\left(\mathscr{B} \mu_{N}^{2 g}\right)$, flat over $S$; but it follows immediately from the example [Chai and Norman 1990, Appendix] that flatness of $\bar{M}_{g}^{(N) \text {,tw }}$ fails in mixed characteristic, even over the ordinary locus of 
$M_{g}$. Of course, if $N$ is invertible on $S$ then this definition is the correct one. More

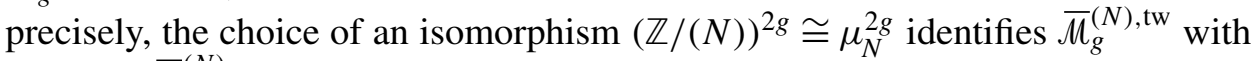
the stack $\bar{M}_{g}^{(N)}$ of [Abramovich et al. 2003, §6], which is shown in [loc. cit.] to be smooth over $\mathbb{Z}[1 / N]$ and proper over $\overline{\mathcal{M}}_{g}$. One would hope to be able to change the above definition to get a closed substack $\bar{M}_{g}^{(N), \text { tw }}$ of $\overline{\mathscr{K}}_{g, 0}\left(\mathscr{B} \mu_{N}^{2 g}\right)$, flat over $S$, agreeing with $\bar{M}_{g}^{(N)}$ over $S[1 / N]$ and with a natural moduli interpretation in terms of the maps from $(\mathbb{Z} /(N))^{2 g}$ to the group schemes $\operatorname{Pic}_{\mathscr{C} / S}^{0}$.

More generally we have good properties for the moduli stack $\overline{\mathscr{K}}_{g, 0}(\mathscr{B} G)$ whenever $G$ is a finite diagonalizable or locally diagonalizable group scheme over $S$, so the Cartier dual $G^{*}$ is commutative and constant or locally constant. Recall that for a finite group $G$ there is a stack ${ }_{G} \mathcal{M}_{g}$ over $\mathbb{Z}[1 /|G|]$ of Teichmüller structures of level $G$ on smooth curves (see [Deligne and Mumford 1969, 5.6; Pikaart and de Jong 1995]). Now if $G$ is a diagonalizable group scheme with $|G|$ invertible on the base scheme $S$, then after adjoining appropriate roots of unity we may fix an isomorphism $G \cong G^{*}$. In [Abramovich et al. 2003, 5.2.3] it is observed that this determines an isomorphism between $G^{*} \mathcal{M}_{g}$ and a substack $\mathcal{B}_{g}^{\text {tei }}(G)^{\circ}$ of $\overline{\mathscr{K}}_{g, 0}^{\circ}(\mathscr{B} G)$ whose closure $\mathcal{B}_{g}^{\text {tei }}(G)$ in $\overline{\mathscr{K}}_{g, 0}(\mathscr{\Re} G)$ is a moduli stack whose geometric objects correspond precisely to $G$-torsors $P \rightarrow \mathscr{b}$ which are connected (where $\mathscr{b}$ is a genus- $g$ twisted stable curve); these are called twisted Teichmüller $G$-structures. One would hope that $\mathcal{B}_{g}^{\text {tei }}(G)$ can be defined in arbitrary characteristic, with a natural moduli interpretation, but it is not clear to the author how to proceed with this for genus $g>1$; as discussed above, it does not suffice to simply consider the substack of $\overline{\mathscr{K}}_{g, 0}(\mathscr{B} G)$ whose geometric objects correspond to $G$-torsors which are connected, since $\mu_{p^{n}}$ is connected in characteristic $p$, and the definition in terms of "full sets of sections" does not give a stack flat over the base scheme in mixed characteristic.

\section{Acknowledgements}

The author is grateful to his advisor, Martin Olsson, for suggesting this research topic and for many helpful conversations, as well as to the reviewer for several helpful comments and corrections. This material is based upon work supported by the National Science Foundation Graduate Research Fellowship Program under Grant No. DGE 1106400.

\section{References}

[Abramovich 2012] D. Abramovich, "Raynaud's group-scheme and reduction of coverings", pp. 1-18 in Number theory, analysis and geometry, edited by D. Goldfeld et al., Springer, New York, 2012. MR 2867909 Zbl 06085939 
[Abramovich and Hassett 2011] D. Abramovich and B. Hassett, "Stable varieties with a twist", pp. 1-38 in Classification of algebraic varieties (Schiermonnikoog, 2009), edited by C. Faber et al., Eur. Math. Soc., Zürich, 2011. MR 2012c:14023 Zbl 1223.14039

[Abramovich and Romagny 2012] D. Abramovich and M. Romagny, "Moduli of Galois $p$-covers in mixed characteristics", Algebra Number Theory 6:4 (2012), 757-780. MR 2966718 Zbl 06064729

[Abramovich and Vistoli 2002] D. Abramovich and A. Vistoli, "Compactifying the space of stable maps”, J. Amer. Math. Soc. 15:1 (2002), 27-75. MR 2002i:14030 Zbl 0991.14007

[Abramovich et al. 2003] D. Abramovich, A. Corti, and A. Vistoli, "Twisted bundles and admissible covers”, Comm. Algebra 31:8 (2003), 3547-3618. MR 2005b:14049 Zbl 1077.14034

[Abramovich et al. 2008a] D. Abramovich, T. Graber, and A. Vistoli, "Gromov-Witten theory of Deligne-Mumford stacks”, Amer. J. Math. 130:5 (2008), 1337-1398. MR 2009k:14108 Zbl 1193. 14070

[Abramovich et al. 2008b] D. Abramovich, M. Olsson, and A. Vistoli, "Tame stacks in positive characteristic", Ann. Inst. Fourier (Grenoble) 58:4 (2008), 1057-1091. MR 2009c:14002 Zbl 1222.14004

[Abramovich et al. 2011] D. Abramovich, M. Olsson, and A. Vistoli, "Twisted stable maps to tame Artin stacks", J. Algebraic Geom. 20:3 (2011), 399-477. MR 2012c:14024 Zbl 1225.14020

[Arbarello et al. 2011] E. Arbarello, M. Cornalba, and P. A. Griffiths, Geometry of algebraic curves, vol. 2, Grundlehren Math. Wiss. 268, Springer, Heidelberg, 2011. MR 2012e:14059 Zbl 1235.14002

[Bourbaki 1998] N. Bourbaki, Éléments de mathématique, algèbre commutative, chapitre 10: Profondeur, régularité, dualité, Masson, Paris, 1998. MR 2007f:13001

[Chai and Norman 1990] C.-L. Chai and P. Norman, "Bad reduction of the Siegel moduli scheme of genus two with $\Gamma_{0}(p)$-level structure", Amer. J. Math. 112:6 (1990), 1003-1071. MR 91i:14033 Zbl 0734.14010

[Conrad 2007] B. Conrad, "Arithmetic moduli of generalized elliptic curves", J. Inst. Math. Jussieu 6:2 (2007), 209-278. MR 2008e:11073 Zbl 1140.14018

[Deligne and Mumford 1969] P. Deligne and D. Mumford, "The irreducibility of the space of curves of given genus", Inst. Hautes Études Sci. Publ. Math. 36:1 (1969), 75-109. MR 41 \#6850 Zbl 0181.48803

[Deligne and Rapoport 1973] P. Deligne and M. Rapoport, "Les schémas de modules de courbes elliptiques", pp. 143-316 in Modular functions of one variable (Antwerp, 1972), vol. 2, edited by P. Deligne and W. Kuyk, Lecture Notes in Mathematics 349, Springer, Berlin, 1973. MR 49 \#2762 Zbl 0281.14010

[Fulton 1998] W. Fulton, Intersection theory, 2nd ed., Ergeb. Math. Grenzgeb. (3) 2, Springer, Berlin, 1998. MR 99d:14003 Zbl 0885.14002

[Katz and Mazur 1985] N. M. Katz and B. Mazur, Arithmetic moduli of elliptic curves, Annals of Mathematics Studies 108, Princeton University Press, 1985. MR 86i:11024 Zbl 0576.14026

[Keel and Mori 1997] S. Keel and S. Mori, "Quotients by groupoids", Ann. of Math. (2) 145:1 (1997), 193-213. MR 97m:14014 Zbl 0881.14018

[Knutson 1971] D. Knutson, Algebraic spaces, Lecture Notes in Mathematics 203, Springer, Berlin, 1971. MR 46\#1791 Zbl 0221.14001

[Petersen 2012] D. Petersen, "Cusp form motives and admissible G-covers", Algebra Number Theory 6:6 (2012), 1199-1221. MR 2968638 Zbl 06126973

[Pikaart and de Jong 1995] M. Pikaart and A. J. de Jong, "Moduli of curves with non-abelian level structure", pp. 483-509 in The moduli space of curves (Texel Island, 1994), edited by R. Dijkgraaf et al., Progress in Mathematics 129, Birkhäuser, Boston, MA, 1995. MR 97e:14037 Zbl 0860.14024 
[Shatz 1986] S. S. Shatz, "Group schemes, formal groups, and p-divisible groups", pp. 29-78 in Arithmetic geometry (Storrs, CT, 1984), edited by G. Cornell and J. H. Silverman, Springer, New York, 1986. MR 861972 Zbl 0603.14033

Communicated by Brian Conrad

Received 2012-08-01 Revised 2013-01-04 Accepted 2013-02-09

andrew@math.berkeley.edu Department of Mathematics, University of California, Berkeley, Berkeley, CA 94720, United States

http://math.berkeley.edu/ andrew/ 


\section{Algebra \& Number Theory}

msp.org/ant

\section{EDITORS}

MANAGING EDITOR

Bjorn Poonen

Massachusetts Institute of Technology

Cambridge, USA

\author{
EDITORIAL BOARD CHAIR \\ David Eisenbud \\ University of California \\ Berkeley, USA
}

\section{BOARD OF EDITORS}

Georgia Benkart

Dave Benson

Richard E. Borcherds

John H. Coates

J-L. Colliot-Thélène

Brian D. Conrad

Hélène Esnault

Hubert Flenner

Edward Frenkel

Andrew Granville

Joseph Gubeladze

Roger Heath-Brown

Ehud Hrushovski

Craig Huneke

Mikhail Kapranov

Yujiro Kawamata

János Kollár

Yuri Manin

Barry Mazur

Philippe Michel
University of Wisconsin, Madison, USA

University of Aberdeen, Scotland

University of California, Berkeley, USA

University of Cambridge, UK

CNRS, Université Paris-Sud, France

University of Michigan, USA

Freie Universität Berlin, Germany

Ruhr-Universität, Germany

University of California, Berkeley, USA

Université de Montréal, Canada

San Francisco State University, USA

Oxford University, UK

Hebrew University, Israel

University of Virginia, USA

Yale University, USA

University of Tokyo, Japan

Princeton University, USA

Northwestern University, USA

Harvard University, USA

École Polytechnique Fédérale de Lausanne
Susan Montgomery

Shigefumi Mori

Raman Parimala

Jonathan Pila

Victor Reiner

Karl Rubin

Peter Sarnak

Joseph H. Silverman

Michael Singer

Vasudevan Srinivas

J. Toby Stafford

Bernd Sturmfels

Richard Taylor

Ravi Vakil

Michel van den Bergh

Marie-France Vignéras

Kei-Ichi Watanabe

Efim Zelmanov

Shou-Wu Zhang
University of Southern California, USA

RIMS, Kyoto University, Japan

Emory University, USA

University of Oxford, UK

University of Minnesota, USA

University of California, Irvine, USA

Princeton University, USA

Brown University, USA

North Carolina State University, USA

Tata Inst. of Fund. Research, India

University of Michigan, USA

University of California, Berkeley, USA

Harvard University, USA

Stanford University, USA

Hasselt University, Belgium

Université Paris VII, France

Nihon University, Japan

University of California, San Diego, USA

Princeton University, USA

PRODUCTION

production@msp.org

Silvio Levy, Scientific Editor

See inside back cover or msp.org/ant for submission instructions.

The subscription price for 2013 is US $\$ 200 /$ year for the electronic version, and $\$ 350 /$ year $(+\$ 40$, if shipping outside the US) for print and electronic. Subscriptions, requests for back issues and changes of subscribers address should be sent to MSP.

Algebra \& Number Theory (ISSN 1944-7833 electronic, 1937-0652 printed) at Mathematical Sciences Publishers, 798 Evans Hall \#3840, c/o University of California, Berkeley, CA 94720-3840 is published continuously online. Periodical rate postage paid at Berkeley, CA 94704, and additional mailing offices.

ANT peer review and production are managed by EditFLOW ${ }^{\circledR}$ from Mathematical Sciences Publishers.

\section{PUBLISHED BY}

- mathematical sciences publishers

nonprofit scientific publishing

http://msp.org/

(C) 2013 Mathematical Sciences Publishers 


\section{Algebra \& Number Theory}

Volume $7 \quad$ No. $9 \quad 2013$

Multiplicities associated to graded families of ideals Steven Dale Cutkosky

Normal coverings of linear groups

JOHN R. BRITNELL and ATTILA MARÓTI

Modularity of the concave composition generating function

George E. ANDrews, Robert C. RhOAdes and SANDER P. ZWEgERS

ANDREW NILES

Regular permutation groups of order $m p$ and Hopf Galois structures

2203

ТIMOTHY KOHL

Further evidence for conjectures in block theory

2241

BENJAMIN SAMBALE

Network parametrizations for the Grassmannian

2275

Kelli TALASKa and LAUREN Williams

Chow quotients of toric varieties as moduli of stable log maps

2313

Qile Chen and Matthew Satriano

Vinberg's representations and arithmetic invariant theory

JACK A. THORNE 